\title{
USE OF MANIFOLDS IN THE INSERTION OF BALLISTIC CYCLER TRAJECTORIES
}

\author{
A Thesis \\ presented to \\ the Faculty of California Polytechnic State University, \\ San Luis Obispo
}

\author{
In Partial Fulfillment \\ of the Requirements for the Degree \\ Master of Science in Aerospace Engineering
}

by

Oliver Morrison

May 2018 
(c) 2018

Oliver Morrison

ALL RIGHTS RESERVED 


\section{COMMITTEE MEMBERSHIP}

TITLE:

Use of Manifolds in the Insertion of Ballis-

tic Cycler Trajectories

AUTHOR:

Oliver Morrison

DATE SUBMITTED: May 2018

COMMITTEe CHAIR: Kira Abercromby, Ph.D.

Associate Professor of Aerospace Engineering

COMmittee MeMBER: Jordi Puig-Suari, Ph.D.

Professor of Aerospace Engineering

COMMitTEE MEMBER: Colleen Kirk, Ph.D.

Professor of Mathematics

COMMITTEe MEMBER: Eric Mehiel, Ph.D.

Professor of Aerospace Engineering 


\begin{abstract}
Use of Manifolds in the Insertion of Ballistic Cycler Trajectories
\end{abstract}

Oliver Morrison

Today, Mars is one of the most interesting and important destinations for humankind and copious methods have been proposed to accomplish these future missions. One of the more fascinating methods is the Earth-Mars cycler trajectory which is a trajectory that accomplishes repeat access to Earth and Mars with little to no fuel-burning maneuvers. This would allow fast travel to and from Mars, as well as grant the possibility of multiple missions using the same main vehicle.

Insertion from Earth-orbit onto the cycler trajectory has not been thoroughly explored and the only existing method so far is a Hohmann-esque transfer via direct burn. The use of manifolds from gravitational equilibrium points has not been considered for low energy transfer to the cycler trajectory. This work is primarily focused on closing this gap and analyzing the feasibility of this maneuver.

To accomplish this, a study of the cycler trajectory - and the S1L1-B class specifically - was completed. The required gravity assist maneuvers at each planet was analyzed through $V_{\infty}$ matching and the entire trajectory was generated over the required inertial period. This method allowed for the generation of 2 cycler trajectories of the inbound and outbound classes, which combine to allow for a reduction in the amount of time the astronauts spend in space.

The Earth-Sun L2 point is analyzed as a potential hub for the maneuver and a halo orbit about this libration point is optimized for low energy transfer from and Earth parking orbit. The associated invariant manifold is then optimized for launch date and distance to the first trajectory on the cycler in order to burn from a trajectory on the manifold to the cycler trajectory. 
The comparisons of this work lie in the required $\Delta V$ to perform each maneuver compared to a direct burn onto the cycler trajectory. These values are compared and the practicality of this maneuver is drawn from these comparisons. It was found that the total required $\Delta V$ for the manifold method is larger than a direct burn from Earth orbit. However, this considers the trajectory from Earth to the halo orbit and if this is removed from consideration the $\Delta V$ is significantly reduced.

It was shown that the feasibility of this method relies heavily on the starting position of the cycler vehicle. If the vehicle begins in Earth-orbit, a direct burn is preferred, however, if the vehicle began in a halo orbit (say it was assembled there) the manifold maneuver is largely preferable. 


\section{ACKNOWLEDGMENTS}

Dedicated to:

- Kira Michelle Jorgensen Abercromby, for her continued dedication and for being my life's inspiration

- Megan Samantha Rund, without her, this thesis would not be possible

- The future astronauts and first settlers of Mars, in the hopes that this project will aid in their expedition. I'll meet you there! 


\section{TABLE OF CONTENTS}

$\begin{aligned} \text { Page } & \end{aligned}$

LIST OF TABLES . . . . . . . . . . . . . . . . . $\quad$ ix

LIST OF FIGURES . . . . . . . . . . . . . . . . . . . . . CHAPTER

1 Introduction . . . . . . . . . . . . . . . . . 1

1.1 The Cycler Trajectory . . . . . . . . . . . . . . . . . 1

1.2 Potential Manifold Solutions . . . . . . . . . . . . . . . . . . 2

1.3 Scope ............................. 2

2 Cycler Trajectory Examination . . . . . . . . . . . . . . . 3

2.1 Background on Cycler Trajectories . . . . . . . . . . . . . 3

2.2 Gravity Assist Maneuvers . . . . . . . . . . . . . . . . 4

2.3 Lambert's Problem . . . . . . . . . . . . . . . . . 6

2.4 Cycler Classification . . . . . . . . . . . . . . . . . . . . . 7

2.5 The "Aldrin Cycler" . . . . . . . . . . . . . . . . . . . . . . . . 10

2.6 The Ballistic S1L1 Cycler . . . . . . . . . . . . . 11

2.6.1 The Circular Solution . . . . . . . . . . . . . . 13

2.6.2 The Ephemeris Model Solution . . . . . . . . . . . . . . . 14

3 Manifold Dynamics Examination . . . . . . . . . . . . . . . . 19

3.1 Background of Manifold Dynamics . . . . . . . . . . . . . 19

3.2 The Circular Restricted 3-Body Problem . . . . . . . . . . . . . . . 20

3.2.1 Libration Points . . . . . . . . . . . . . . . 23

3.3 Halo Orbits . . . . . . . . . . . . . . . . . . 25

3.4 Manifolds ....................... 26

4 Cyclers . . . . . . . . . . . . . . . . . . . . . 29

4.1 Verifying The Ephemeris S1L1-B Cycler . . . . . . . . . . . . . . 29

4.1.1 Pork-Chop Plots . . . . . . . . . . . . . . 30

4.2 Cycler Generation . . . . . . . . . . . . . . . . . . 35

4.2.1 Transfer Times . . . . . . . . . . . . . . . 35

4.2.2 Relative Positions . . . . . . . . . . . . . . . . . . . 36 
4.2 .3 The Search . . . . . . . . . . . . . . . . . . . . . 37

4.3 Insertion $\Delta V \ldots \ldots \ldots \ldots \ldots \ldots$

5 Manifolds . . . . . . . . . . . . . . . . . . . . 45

5.1 Halo Orbit Insertion . . . . . . . . . . . . . . . . . 45

5.1 .1 Halo Orbit Optimization . . . . . . . . . . . . . . 48

$5.2 \quad$ Halo Orbit Departure . . . . . . . . . . . . . . . . . . . 54

5.2 .1 Time of Departure . . . . . . . . . . . . . . . 54

6 Discussion of Various Test Cases _. . . . . . . . . . . . . . 66

6.1 Nominal Cases . . . . . . . . . . . . . . . . . . 66

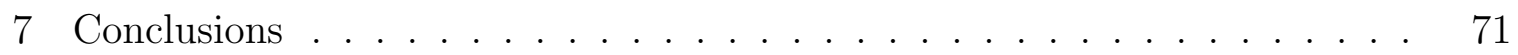

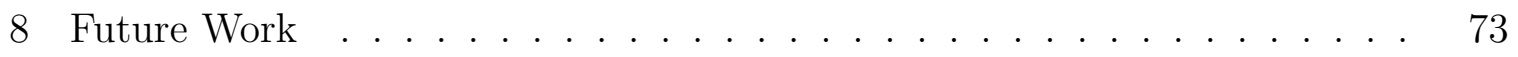

8.1 Cycler Trajectories . . . . . . . . . . . . . . . 73

8.2 Manifolds . . . . . . . . . . . . . . . . . . . . . . 74

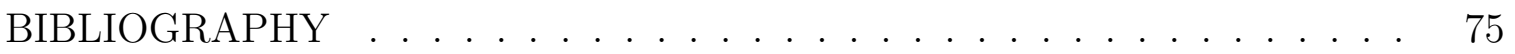
APPENDICES

A Cycler Trajectory Extras . . . . . . . . . . . . . . 77

A.1 Aldrin Cycler Computation _. . . . . . . . . . . . 77

A.2 S1L1-B Cycler Computation (Circular Model) . . . . . . . 78

A.3 Extra Itineraries $\ldots \ldots \ldots \ldots$

A.4 Timeline Characteristics $\ldots \ldots \ldots \ldots \ldots$

B Manifold Extras . . . . . . . . . . . . . . . . . 85

B.1 Synodic Frame Test Case Plots . . . . . . . . . . . 85 


\section{LIST OF TABLES}

Table

Page

2.1 Example Outbound Cycler Vehicle Itinerary (T. Troy McConaghy) 17

4.1 Transfer times for inbound and outbound cyclers . . . . . . . . 36

4.2 Relative angular positions of Earth and Mars . . . . . . . . . . 37

4.3 Generated outbound cycler trajectory itinerary . . . . . . . . . . . 40

4.4 Generated inbound cycler trajectory itinerary . . . . . . . . . . 42

5.1 Optmization results for various halo orbit regimes . . . . . . . . 50

5.2 Table of example output values for frame rotation . . . . . . . . 60

6.1 Manifold to cycler insertion results for various vehicles . . . . . . 68

A.1 Example Inbound Cycler Itinerary (T. Troy McConaghy) . . . . . . 81

A.2 Second generated outbound cycler trajectory itinerary . . . . . . . 82 


\section{LIST OF FIGURES}

Figure

Page

2.1 Flyby visualization for general system . . . . . . . . . . 5

2.2 Shape of Lambert's solution with varying parameters . . . . . . . 8

2.3 The Aldrin Cycler over two transfers . . . . . . . . . . . . . . . . . 12

2.4 The S1L1 Cycler first 2 transfers (Circular/co-planar model) . . . . 15

2.5 The Ballistic S1L1 Outbound Cycler first two legs (Ephemeris Model) 18

3.1 Circular Restricted 3-Body Problem Geometry . . . . . . . . . . . 21

3.2 Libration point locations for generic system . . . . . . . . . . 24

3.3 Various halo orbits about Earth-Sun L1 . . . . . . . . . . 26

3.4 Manifolds approaching and departing L1 halo . . . . . . . . . . 28

4.1 Pork-chop plot for Earth-Mars transfer . . . . . . . . . . . . . . 31

4.2 Pork-chop plot for Mars-Earth transfer . . . . . . . . . . . . . . . 31

$4.3 V_{\infty}$ surface plot for Earth-Mars-Earth trajectory . . . . . . . . . 33

4.4 Generated timeline from known itinerary . . . . . . . . . . . . 34

4.5 First 3 legs of generated outbound cycler . . . . . . . . . . . 41

$4.6 \quad$ First 3 legs of generated inbound cycler . . . . . . . . . . . 43

5.1 Example optimized trajectory for given halo orbit . . . . . . . . 47

5.2 Parking orbit to trajectory in manifold . . . . . . . . . . 47

5.3 Trajectory in manifold in sun-centered inertial frame . . . . . . . . 48

$5.4 \quad$ Optimization example for $A_{z}=7 * 10^{5} \mathrm{~km}$. . . . . . . . . . . . 50

5.5 Optimized halo orbit and stable manifold trajectory . . . . . . . 52

5.6 Optimized solution parking orbit and trajectory . . . . . . . 53

5.7 Earth and Mars circular vs. ephemeris orbit shapes . . . . . . . 55

5.8 Unstable manifold over $6 \mathrm{TU}$ in inertial frame . . . . . . . . . . 57

5.9 Unstable Manifold over $6 \mathrm{TU}$ in fixed frame . . . . . . . . . . 58

5.10 Example of unstable manifold trajectory . . . . . . . . . . 61

5.11 First halo optimization $\mid \theta_{z}=(0,2 \pi) \ldots \ldots . \ldots 2$ 
5.12 First halo optimization $\mid \theta_{z}=(-\pi, \pi) \ldots \ldots \ldots \ldots \ldots \ldots$

5.13 Entire trajectory from parking orbit to cycler insertion in synodic frame .......................... 64

5.14 Unstable manifold trajectory to cycler insertion in inertial frame . . 65

6.1 Inbound vehicle 1 results . . . . . . . . . . . . . . 67

6.2 Outbound vehicle 2 results . . . . . . . . . . . . . . . 68

6.3 Inbound vehicle 2 results . . . . . . . . . . . . . . . . . . . . 69

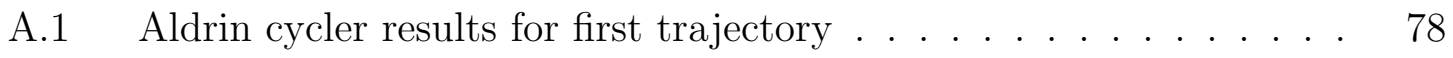

A.2 S1L1-B results for legs 1 and $2 \ldots \ldots \ldots \ldots$

A.3 Intersection of $V_{\infty}$ contour on timeline $\ldots \ldots \ldots \ldots$

A.4 Double intersection of $V_{\infty}$ contour on timeline $\ldots \ldots \ldots \ldots$

B.1 Trajectory in synodic frame for Inbound -1 vehicle . . . . . . . . 86

B.2 Trajectory in synodic frame for Outbound-2 vehicle . . . . . . 86

B.3 Trajectory in synodic frame for Inbound-2 vehicle . . . . . . . . 87 
Chapter 1

\section{INTRODUCTION}

\subsection{The Cycler Trajectory}

For decades, Mars has been the source of human fascination and is currently the next destination for today's astronauts. Currently, the most pressing issue with getting to the red planet lies in it's sheer difficulty. This is especially apparent when considering crewed missions and the associated $\Delta V$ cost. Several remedies have been proposed for this issue and among the most popular is the Earth-Mars cycler, first introduced by Buzz Aldrin in 1985[9]. Through gravity assist maneuvers and repeat visits to Earth and Mars, this type of trajectory would allow several several crews over many years to visit Mars with only one main transport vehicle and little to no $\Delta V$ cost throughout its course. Many cyclers since the introduction of the "Aldrin Cycler" have been proposed with the most notable (and practical) being the S1L1-B cycler[14].

Though these trajectories are very effective in reducing overall mission cost and lifetime $\Delta V$, cycler insertion is still expensive in many respects. The vehicle in question would likely be very large to accommodate a long lifetime, a habitat for the crew, as well as multiple types of cargo; which would mean launching from the ground directly into transfer would be impractical. Furthermore, the insertion of cycler trajectories has not been thoroughly examined, and would thusly required a direct burn transfer. Therefore, a rectification to the cycler insertion is necessary in order to remedy some of these potential issues. 


\subsection{Potential Manifold Solutions}

This work explores the potential of using manifolds from halo orbits in the Earth-Sun system in order to reduce the mission cost, effectively making the cycler trajectory a more practical option for crewed missions to Mars. Under the assumption that the vehicle would start in a predetermined parking orbit, the vehicle could do a series of relatively small burns to enter a halo orbit around Earth-Sun L2, perturb its orbit slightly to enter onto a trajectory in the associated manifold, and then perform a final burn to enter the desired first leg of the cycler trajectory (after some time in the manifold).

By greatly reducing the $\Delta V$ cost of the first transfer (compared to a normal Hohmann transfer) the practicality of a large vehicle designed for a large mission lifetime is substantially increased. Furthermore, because the velocities of objects on manifolds are very slow compared to the celestial speed of our solar system, the supplementary advantage of additional time to perform maintenance or further planning may be available if necessary.

\subsection{Scope}

The scope of this work is aimed solely at determining the validity of this type of transfer as well as explore the dynamics of cycler trajectories in order to gain a better

understanding of them. This work will be limited to S1L1-B cycler trajectories, and only the dynamics around Earth-Sun L2 will be considered during the manifold phase. Further work will be discussed in the appropriately named section. An analysis will also be made about the total $\Delta V$ mission savings. 
Chapter 2

\section{CYCLER TRAJECTORY EXAMINATION}

In this chapter, the underlying concepts, background, and various computation methods of cycler trajectories will be succinctly analyzed in order to become more familiar with their properties before they are analyzed in greater detail later in this work.

\subsection{Background on Cycler Trajectories}

In the early 1980s, famous astronaut and engineer Buzz Aldrin began working on his concept of a Lunar transport system that would allow routine measures, such as maintenance, supplies, or crewed missions, to be a more repeatable process to the Moon[1]. Eventually dubbed the "Lunar Cycler" this process was deemed unfit for further analysis because of the largely increased transfer time between the Earth and Moon. Later in 1985, Aldrin began extending his work however, to the Earth-Mars system as a more practical approach to his solution. He then created what is now called the "Aldrin Cycler"[1].

The concept of a cycler can be simply summarized as: any trajectory with repeated access to two or more bodies in a system. Many cycler trajectories have been studied (or proposed) with some of the more notable being,

- Earth-Moon or Lunar Cyclers

- Earth-Mars Cyclers

- Earth-Venus Cyclers

For the purposes of this project, the Earth-Mars cycler will be the focal point of study as it is the most studied and the most exciting in terms of future human developments 
in space. Many variations of this trajectory exist and thusly the computation and shape of them differs widely. A few of the methods and visualizations of their shapes will be analyzed later in the chapter; additionally, a detailed computation of these can be found in Appendix A.

Since the formulation of the Aldrin cycler, many other Earth-Mars cycler trajectories have been discovered and analyzed. In a paper by a team at the University of Texas in Austin[18], many different classes of cyclers are analyzed and compared for the purposes of generating a solution space. Though many of these trajectories are impractical for human exploration, they do allow for further understanding of what is required to generate and optimize them.

Following these developments, a team from Purdue University was able to take these concepts and polish the cycler trajectory for human exploration purposes, generating the S1L1-B cycler[13]. This specific cycler will be used throughout this work as a running example. Further investigative or applicative work of the cycler has been sparse for many years since 2002 when the S1L1-B was generated; however, these trajectories still prove vital in the quest for human exploration to mars.

\subsection{Gravity Assist Maneuvers}

The fundamental process by which cycler trajectories operate, especially those in the ballistic class, are gravity assist maneuvers, otherwise known as flybys. Using the gravitational forces of the planet alone, it is possible to perform a rotation of the heliocentric (sun-centered) orbital trajectory. This is extremely useful in applications that require very complicated patched-conic trajectories, as well as those that require the spacecraft to move large distances. Some of the most notable missions that made use of the flyby are Voyager I \& II[5], Cassini[2], and New Horizons[4]. Cycler trajectories take full advantage of this maneuver to allow for repeat access between 


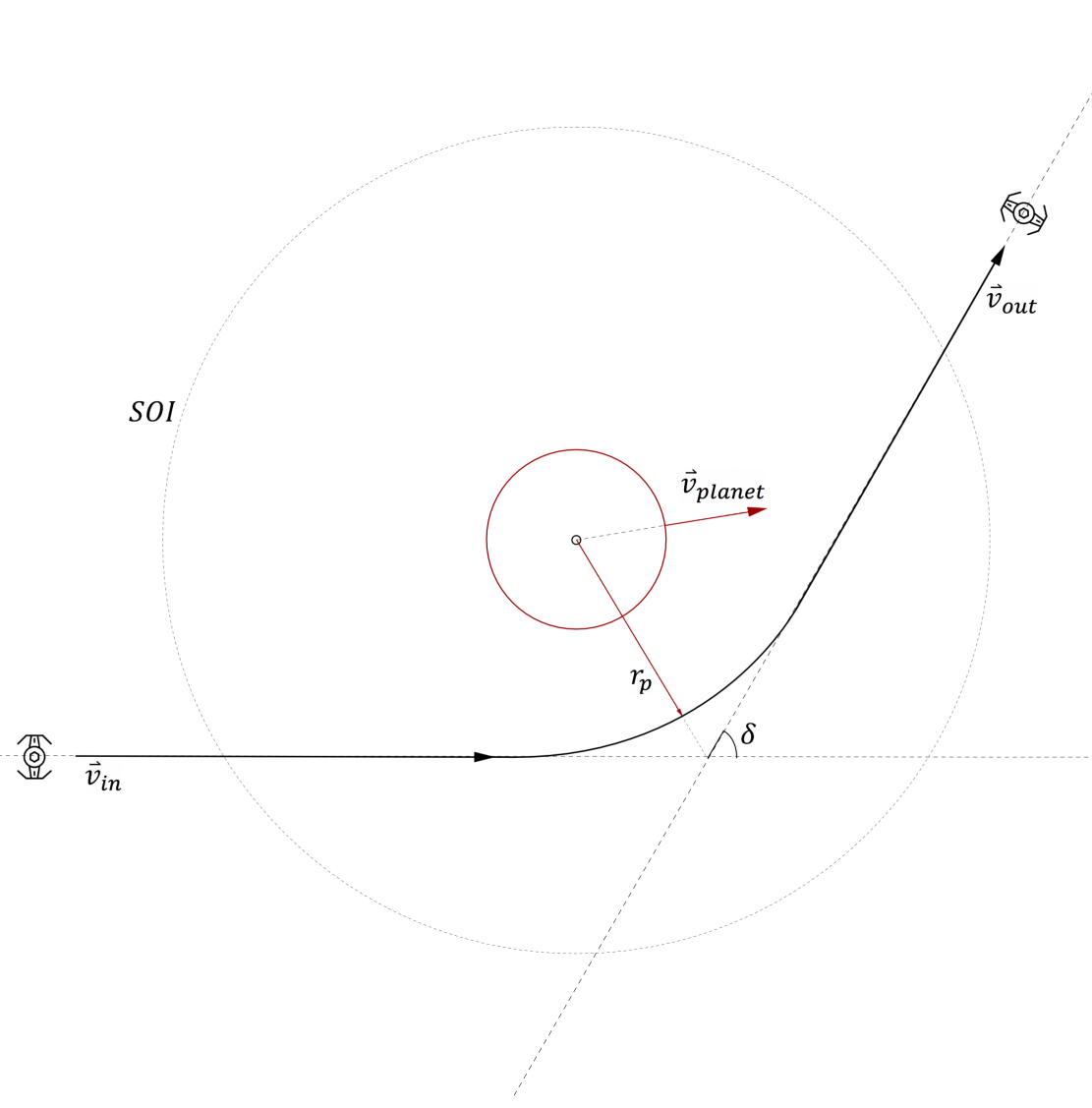

\section{Figure 2.1: Flyby visualization for general system}

Earth and Mars.

The rotation of the orbit occurs as a rotation of the velocity vector of the spacecraft on the incoming trajectory towards the planet. The rotation of the vector can only occur in the region known as the sphere of influence (SOI), which is the approximate distance from the planet that its gravity will still have an effect on the spacecraft. Once inside the SOI, the velocity vector rotates by an angle, $\delta$, known as the turn angle. A diagram to visualize the effects and principles of a flyby are given in Fig. 2.1 .

The turn angle is determined by a few parameters; the first of which is called the hyperbolic excess velocity, or $v_{\infty}$. Although usually represented as a magnitude, 
the vector is simply the difference in the velocity of the spacecraft, $\vec{v}$, to the velocity of the planet, $\vec{v}_{\text {planet }}$, in the heliocentric reference frame. The next parameter is the flyby radius, $r_{p}$ which is the closest distance to the planet that the spacecraft will experience. The last parameter is the standard gravitation parameter, $\mu_{p}$ of the planet itself. This is simply the product of the gravitational constant, $G$, with the mass of the planet. The derivation will be spared here, but two equations for the turn angle are represented by the following :

$$
\begin{aligned}
& \delta=2 \arcsin \frac{\mu_{p}}{\mu_{p}+r_{p} v_{\infty}^{2}} \\
& \delta=\arctan 2\left(\frac{\left\|\vec{v}_{\text {in }} \times \vec{v}_{\text {out }}\right\|}{\left\|\vec{v}_{\text {in }}\right\|\left\|\vec{v}_{\text {out }}\right\|}, \frac{\vec{v}_{\text {in }} \cdot \vec{v}_{\text {out }}}{\left\|\vec{v}_{\text {in }}\right\|\left\|\vec{v}_{\text {out }}\right\|}\right)
\end{aligned}
$$

where, $\arctan 2$ is the multi-value inverse tangent function that allows for quadrant correction. It is also possible to rearrange both equations to solve for $r_{p}$; more on that very soon. If the desired $\vec{v}_{i n}$ and $\vec{v}_{\text {out }}$ are known, the flyby is only ballistic (does not require an extra maneuver) if the incoming and outgoing magnitudes of the hyperbolic excess velocity are equivalent. That is to say

$$
\left\|\vec{v}_{\infty \text { out }}\right\|-\left\|\vec{v}_{\infty \text { in }}\right\|=0
$$

Sometimes, the turn angle is not sufficient to allow for this, as the required $r_{p}$ is smaller than the radius of the planet. In this case, extra fuel must be spent to make up for the total $\Delta V$ required/desired. This is known as a powered flyby and will actively be avoided for the purposes of this project. Furthermore, a method developed for this work for determining ballistic flybys will be presented in Chapter 4 .

\subsection{Lambert's Problem}

One of the oldest and classic problems in orbital mechanics is Lambert's problem. The problem statement is simple: given two orbital position vectors and a transfer time between them, can we find the velocity vectors at each position? Many, many 
solutions have been developed for this problem with varying levels of speed and robustness. However, they all rely on an iterative method as the exact solution can not be found analytically.

For the direction of this work, I have chosen to use a method developed by Izzo \&

Gooding, with elements borrowed from a method by Lancaster \& Blanchard [11]. This method was chosen because it is very fast and is also capable of multi-rev solutions, which are solutions that span more than one orbital period.

Other than multi-rev solutions, there are a few other characteristic inputs that can determine the shape of the solution. Chiefly, these are the short \& long way and left \& right branch solutions. Short and long way simply determine which direction around the sun the orbital path will take, while left and right branch determines the shape of the multi-rev solution. A diagram illustrating how these parameters affect the solution is given in Fig. 2.2. The start and end points are both positions of Earth but only the right branch multi-rev solution matches Earth's orbit.

The most important use of the solution to Lambert's problem for this thesis is to be able to determine the encounter times of Earth and Mars to connect the flyby maneuvers necessary to complete the cycler trajectory, as well as the shape of the cycler trajectory itself. Using the ephemeris model of the solar system, the position and velocity vectors of each planet are known at any time; which, when coupled with a chosen time of flight, a trajectory between Earth and Mars can be determined.

\subsection{Cycler Classification}

Before examining a cycler's computation, its nomenclature will first be momentarily discussed. There are two ways to classify a cycler trajectory, depending on its characteristics. The first belongs to cyclers that contain no intermediate or "taxi" gravity assists or flybys and simply go from one planet to the next. This designation takes 


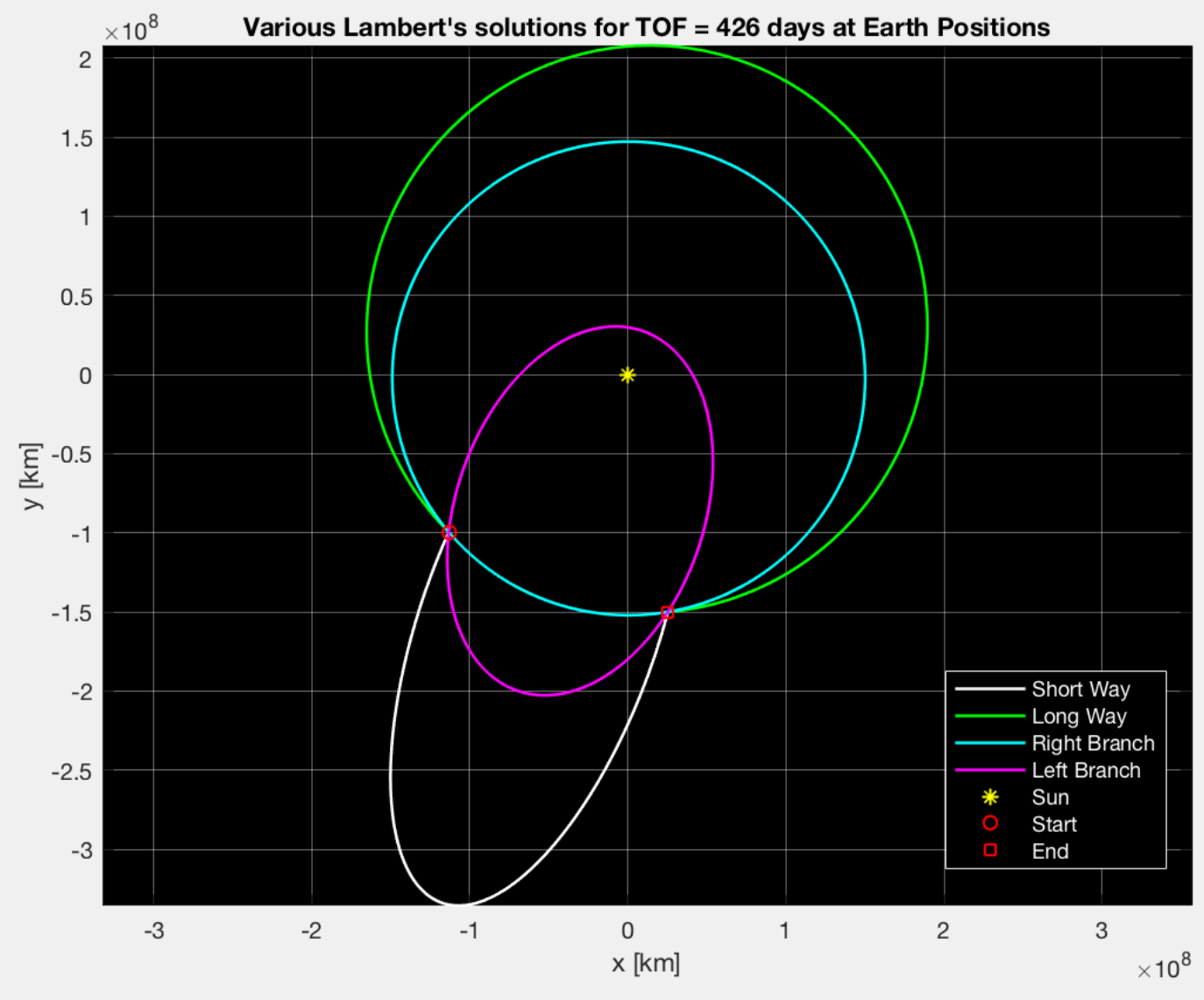

Figure 2.2: Shape of Lambert's solution with varying parameters 
the form $n \mathrm{P} r$, where $n$ is the number of synodic periods until repetition, $\mathrm{P}$ is the period (transfer) type, and $r$ is the number of revolutions (rounded to the nearest integer) in that period. The three period "types" are 'S', 'L', and 'U', for the short, long, and unique period classes respectively. For example, the "Aldrin Cycler", which will be discussed later, is designated to the 1L1 class.

More recently identified cyclers now involve one or more of what may be called a 'taxi Earth flyby' which concerns extra, intermediate, flybys at Earth, thus creating a new trajectory between Earth and itself. These are mainly used in order to achieve ballistic status which will be discussed later. These classes are defined by their transfer between Earth and Mars, followed by Mars to Earth, nominated as Leg 1 and Leg 2. In general, the designation of a specific cycler takes the form $\mathrm{P}_{1} r_{1} \mathrm{P}_{2} r_{2}$, where $\mathrm{P}$ and $r$ share their previous descriptions and the subscripts denote the first and second legs respectively. An example of this type of trajectory is the S1L1 cycler which will be used throughout this project as the working example.

Beyond this, cyclers can also be defined by how fast they operate between planets, known as outbound and inbound. An outbound cycler is one that has a 'quick' trajectory from Earth to Mars while an inbound is quick from Mars to Earth. Both inbound and outbound trajectories are necessary for a feasible crewed mission to Mars as the expedition there and back would need to be short due to space environment concerns. This work will discuss the insertion into both inbound and outbound trajectories since they are both necessary.

As a final appendage, cyclers can also be defined as powered or ballistic. As briefly discussed with the flybys, this designation simply implies whether or not extra $\Delta V$ is required at each gravity assist in order to continue on the proper trajectory. For the direction of this work, a ballistic cycler will be analyzed in order to remain consistent with the overall goal of reducing mission $\Delta V$. 


\subsection{The "Aldrin Cycler"}

When the concept of these types of trajectories was first being considered, many assumptions had to be introduced in order to simplify the problem as a proof of concept rather than a practicality. Buzz Aldrin pioneered this work and his assumptions can be summarized as follows:[19]

- The Earth-Mars synodic period, $S$, is exactly $2 \frac{1}{7}$ years

- The orbits of Earth, Mars, and the cycler lie in the same plane

- The orbits of Earth and Mars are circular

- Gravity assist maneuvers can only occur around Earth

- Gravity assist maneuvers are instantaneous rotations

- $\Delta V$ maneuvers may be required during gravity assist maneuvers

The Aldrin cycler has been explored in some detail and a few methods exist for calculating the trajectory. The following method that was advanced for this work (see Appendix A) will be briefly elucidated here. Under the assumption that at $t=0$ the position of Earth can be written in flat ( $x-y$ plane only), Cartesian coordinates, the circular assumption allows the position of Earth at at any time, $t$, to be written as $[\cos (2 \pi t), \sin (2 \pi t)]$. Thus, the required position of Earth at encounter events can be written as:

$$
\begin{aligned}
R_{t=0} & =[1,0] \\
R_{t=n S} & =[\cos (2 \pi n S), \sin (2 \pi n S)]
\end{aligned}
$$

where, $\mathrm{n}$ carries the designation from the $n \mathrm{P} r$ cycler class and represents the number of periods until the next Earth encounter occurs. The position of Earth at any encounter 
can be solved for depending on the amount of periods to repeat are desired; in this case, $n=1$. This automatically designates the shape of the orbit which can be found easily through a multi-rev Lambert's solver, where $t=0$ and $t=2 \frac{1}{7}$ are the Earth encounter times. The required positions of Mars at its encounter times can then be calculated by analyzing where the cycler trajectory and Mars' orbit intersect.

A visual representation of the Aldrin cycler can be seen in Fig. 2.3. The numbers, $1-4$, in each Earth and Mars correspond to the chronological order of the event of a Martian encounter or Earth flyby. For the sake of brevity in the following explanation, the positions of both planets will be referred to by this number. The vehicle begins at Earth 0 and performs a transfer to Mars 1 in about 147 days (142 sols). No gravity assist occurs at Mars and the vehicle continues for another 633 days (614 sols) until Earth 2 and performs a flyby as well as any additional required $\Delta V$ maneuver. This process is dubbed Transfer 1 and takes a total of 779 days (748 sols) or $2 \frac{1}{7}$ years. The vehicle then repeats this process indefinitely, beginning with Transfer 2 , where the spacecraft moves from Earth 2 to Mars 3 and so on.

Though this specific cycler is useful as a case study for verifying the possibility of this type of transfer, the corpulent assumptions do not allow for a real-world application. This necessitates a new cycler that can operate in an environment that does not require these limiting assumptions. Furthermore, this cycler always requires extra burns at each flyby in order to obtain the proper turn angle. This is an undesirable result that drastically increases mission cost.

\subsection{The Ballistic S1L1 Cycler}

The S1L1 class represents a short, 1-rev period to Earth, followed by a long, 1-rev period to Earth, (since we are using the $\mathrm{P}_{1} r_{1} \mathrm{P}_{2} r_{2}$ designation). As a quick note, the short trajectory to Earth does not contain a Martian encounter. This cycler was first 


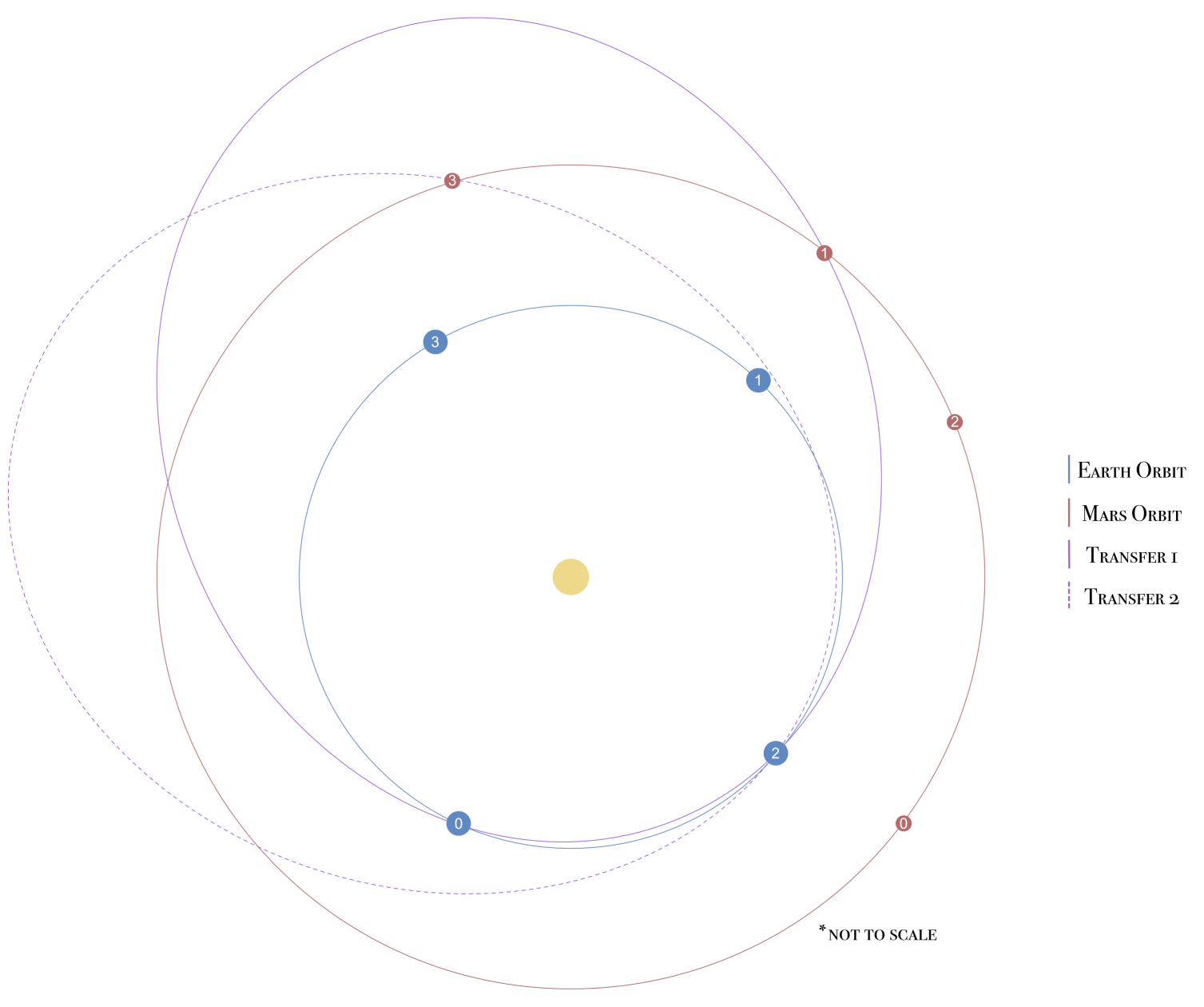

Figure 2.3: The Aldrin Cycler over two transfers 
introduced in the circular/co-planar model but can also be carried to the ephemeris model of the solar system as was shown by McConaghy et al.[13]. For our purposes, both the circular and ephemeris model solutions will be introduced, but the focus will lie more heavily on the ephemeris model solution. Furthermore, this cycler was chosen as a case study principally because of its capacity to perform ballistically, i.e. there are no corrective $\Delta V$ maneuvers over the mission lifetime.

\subsubsection{The Circular Solution}

This solution refined here by the author draws heavily from the process used previously for the Aldrin Cycler (see Appendix A), but was first introduced by McConaghy et al.[13]. Since an intermediate Earth encounter has been added, the encounter time positions can now be written as

$$
\begin{aligned}
R_{t=0} & =[1,0] \\
R_{t=\tau} & =[\cos (2 \pi \tau), \sin (2 \pi \tau)] \\
R_{t=n S} & =[\cos (2 \pi n S), \sin (2 \pi n S)]
\end{aligned}
$$

where $\tau$ is the intermediate Earth encounter time. It is important to note that in this case $n$ is now equal to 2 as this is a multi-period solution. Furthermore, the time between 0 and $\tau$ is referred to as $\operatorname{Leg} 1$, while $\operatorname{Leg} 2$ refers to the time between $\tau$ and $n S$. Unlike the Aldrin Cycler solution, all possible values of $\tau \in(S, 2 S)$ are evaluated, thus removing some of the simplicity and restrictions from the method. The transfer from $\tau \rightarrow 2 S$ is simply an Earth-Earth trajectory and is not required to cross the orbital path of Mars.

The required extra $\Delta V$ at each flyby of Earth is calculated and solutions with values outside of a certain tolerance are removed from consideration. Trajectories which allow for no $\Delta V$ maneuvers are set aside for further consideration and are put in the ballistic class. As an example, the results of a ballistic solution found for 
$\tau=2.8276$ years are as follows:

$$
\begin{aligned}
R_{t=0} & =[1,0] \\
R_{t=2.8276} & =[0.4690,-0.8832] \\
R_{t=4.2857} & =[-0.2225,0.9749]
\end{aligned}
$$

It is important to reiterate that these are the positions of Earth at the cycler vehicles encounter times with Earth. The encounter positions and times of Mars are calculated later just as with the Aldrin Cycler. A diagram of the S1L1 trajectory, similar to the Aldrin cycler, is given in Fig. 2.4. For the sake of clarity, the trajectory will be briefly explained in detail. The vehicle begins on Transfer 1 at Earth 0 and begins a transfer to Mars 1. No flyby occurs at Mars and the vehicle continues to Earth 2 where a flyby is performed and the trajectory is shifted. The vehicle then moves to Earth 3 and performs a final flyby after which the entire process recurs in Transfer 2 and so on.

\subsubsection{The Ephemeris Model Solution}

In order to perform this analysis, some of the previously limiting assumptions, such as circular/co-planar orbits, had to be relaxed. The new assumptions, or rather constraints, used can be summarized as follows:

- Earth and Mars orbits are modeled using JPL's 405 Ephemerides[7] model

- Gravity assist maneuvers can occur at both Earth and Mars

- Gravity assist maneuvers are modeled as instantaneous rotations

Because this model no longer contains some of the niceties from the previous solutions, the approach to the Aldrin cycler or S1L1 circular solution cannot be taken. 


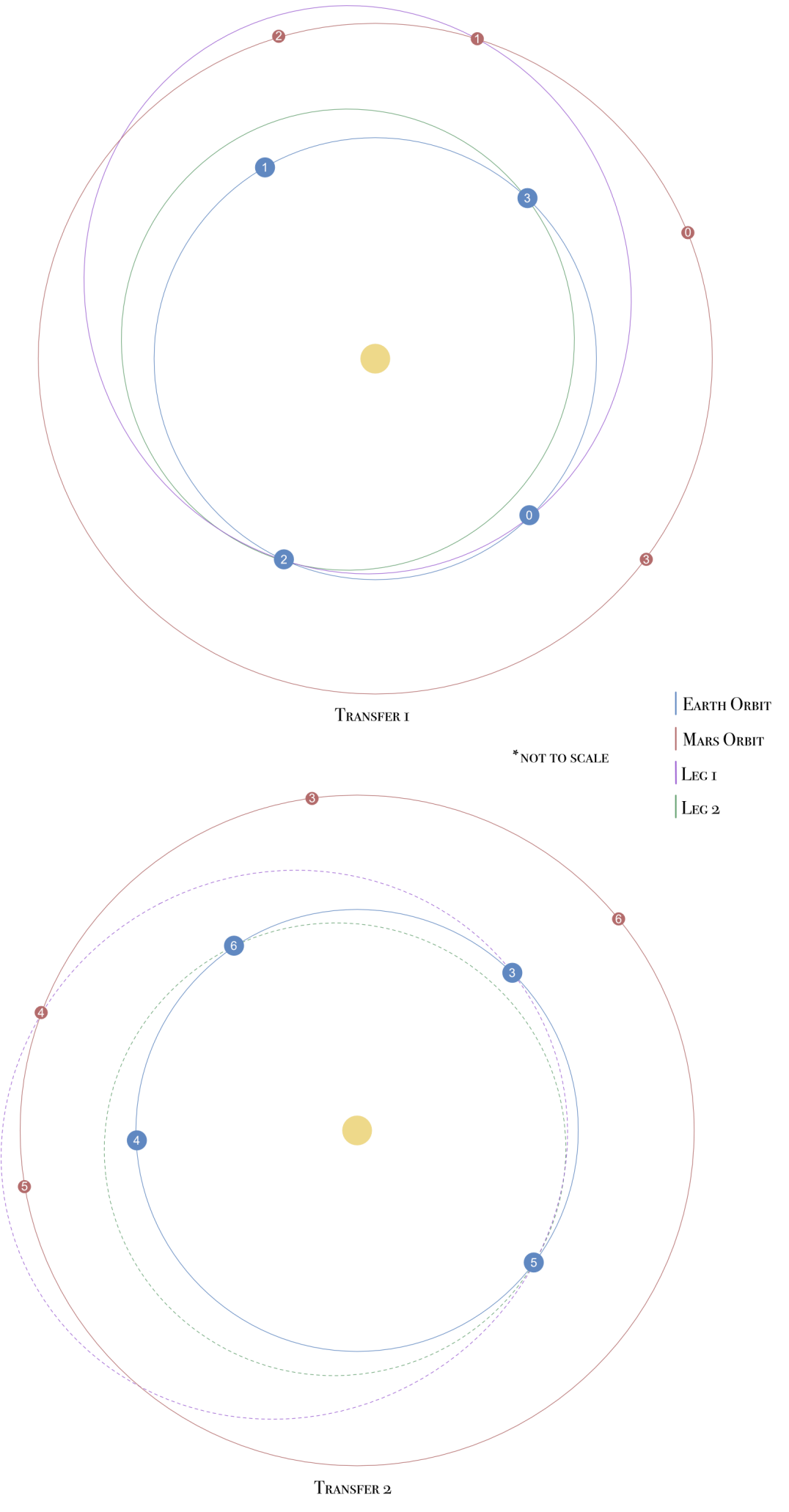

Figure 2.4: The S1L1 Cycler first 2 transfers (Circular/co-planar model) 
Instead, a system of inequalities is optimized in order to converge to a solution. It is important to note that unlike the Aldrin cycler, each 1st leg is now different from the last, so all legs must be solved for ad infinitum (or at least until the positions of Earth and Mars inertially return to their initial states). This return time is $\sim 33$ years and will be hereafter referred to as the Inertial Period. A method presented by McConaghy et al.[14] describes the use of the SNOPT optimizer to generate a trajectory of this caliber, and a self-developed method of verification and generation of these trajectories will be presented later. The SNOPT optimizer stands for Sparse Nonlinear OPTimizer and uses a quasi-newton method of optimization[8].

Because every leg of this solution varies, the simplistic and concise nomenclature of encounter times and positions of Earth cannot be used to represent the trajectory. Instead, over the course of the entire inertial period, all encounter times of both planets are listed (rather than only those at Earth), as well as the flyby altitude and corresponding $v_{\infty}$. The positions of Mars are added since gravity assist maneuvers are now allowed for this planet. This list is known as the cycler itinerary. An example itinerary for an outbound cycler, which will be used extensively throughout this work, is given in Table 2.1. It is important to note that this itinerary was provided by $\mathrm{T}$. Troy McConaghy[15] (along with an inbound example) and was not calculated by the author. However, these will be used a basis for developing new ephemeris solutions later on.

A visualization of the first portion of this trajectory can be seen in Fig. 2.5. Since flybys are allowed at Mars, Leg 1 is differentiated into Leg 1 and Leg 1.5 to account for the fact that although the trajectory changes slightly, they are still portions of the same leg. 
Table 2.1: Example Outbound Cycler Vehicle Itinerary (T. Troy McConaghy)

\begin{tabular}{|c|c|c|c|c|c|c|c|}
\hline \multicolumn{8}{|c|}{ Outbound Cycler Vehicle (Troy McConaghy) } \\
\hline Encounter & Fly-By Planet & Year & Month & Day & $V_{\infty}$ & $\begin{array}{l}\text { Closest } \\
\text { Approach } \\
\text { Distance }\end{array}$ & Leg, TOF \\
\hline$\#$ & $P$ & yyyy & $\mathrm{mm}$ & $d d$ & $\mathrm{~km} / \mathrm{s}$ & $k m$ & days \\
\hline 0 & Earth & 2005 & 8 & 13 & 4.01 & -- & -- \\
\hline 1 & Mars & 2006 & 2 & 27 & 3.02 & 4816 & 198 \\
\hline 2 & Earth & 2008 & 6 & 9 & 6.89 & 20130 & 833 \\
\hline 3 & Earth & 2009 & 12 & 3 & 6.9 & 31110 & 541 \\
\hline 4 & Mars & 2010 & 6 & 6 & 4.31 & 17710 & 186 \\
\hline 5 & Earth & 2012 & 8 & 24 & 6.42 & 26490 & 809 \\
\hline 6 & Earth & 2014 & 2 & 14 & 6.43 & 41520 & 539 \\
\hline 7 & Mars & 2014 & 7 & 3 & 7.14 & 12190 & 138 \\
\hline 8 & Earth & 2016 & 12 & 9 & 4.01 & 27730 & 890 \\
\hline 9 & Earth & 2018 & 5 & 22 & 4.03 & 19920 & 530 \\
\hline 10 & Mars & 2018 & 9 & 15 & 6.47 & 11580 & 115 \\
\hline 11 & Earth & 2021 & 4 & 6 & 4.61 & 22990 & 934 \\
\hline 12 & Earth & 2022 & 9 & 20 & 4.59 & 14780 & 532 \\
\hline 13 & Mars & 2023 & 5 & 1 & 2.77 & 7601 & 223 \\
\hline 14 & Earth & 2025 & 7 & 2 & 7.08 & 23860 & 793 \\
\hline 15 & Earth & 2026 & 12 & 26 & 7.09 & 35120 & 542 \\
\hline 16 & Mars & 2027 & 6 & 14 & 5.27 & 13840 & 170 \\
\hline 17 & Earth & 2029 & 9 & 21 & 5.8 & 26850 & 830 \\
\hline 18 & Earth & 2031 & 3 & 12 & 5.8 & 37520 & 537 \\
\hline 19 & Mars & 2031 & 7 & 15 & 7.85 & 8802 & 125 \\
\hline 20 & Earth & 2034 & 1 & 15 & 4.21 & 24870 & 915 \\
\hline 21 & Earth & 2035 & 6 & 28 & 4.2 & 2756 & 529 \\
\hline 22 & Mars & 2035 & 11 & 12 & 5.87 & 1770 & 137 \\
\hline 23 & Earth & 2038 & 5 & 5 & 7.23 & -- & 906 \\
\hline
\end{tabular}




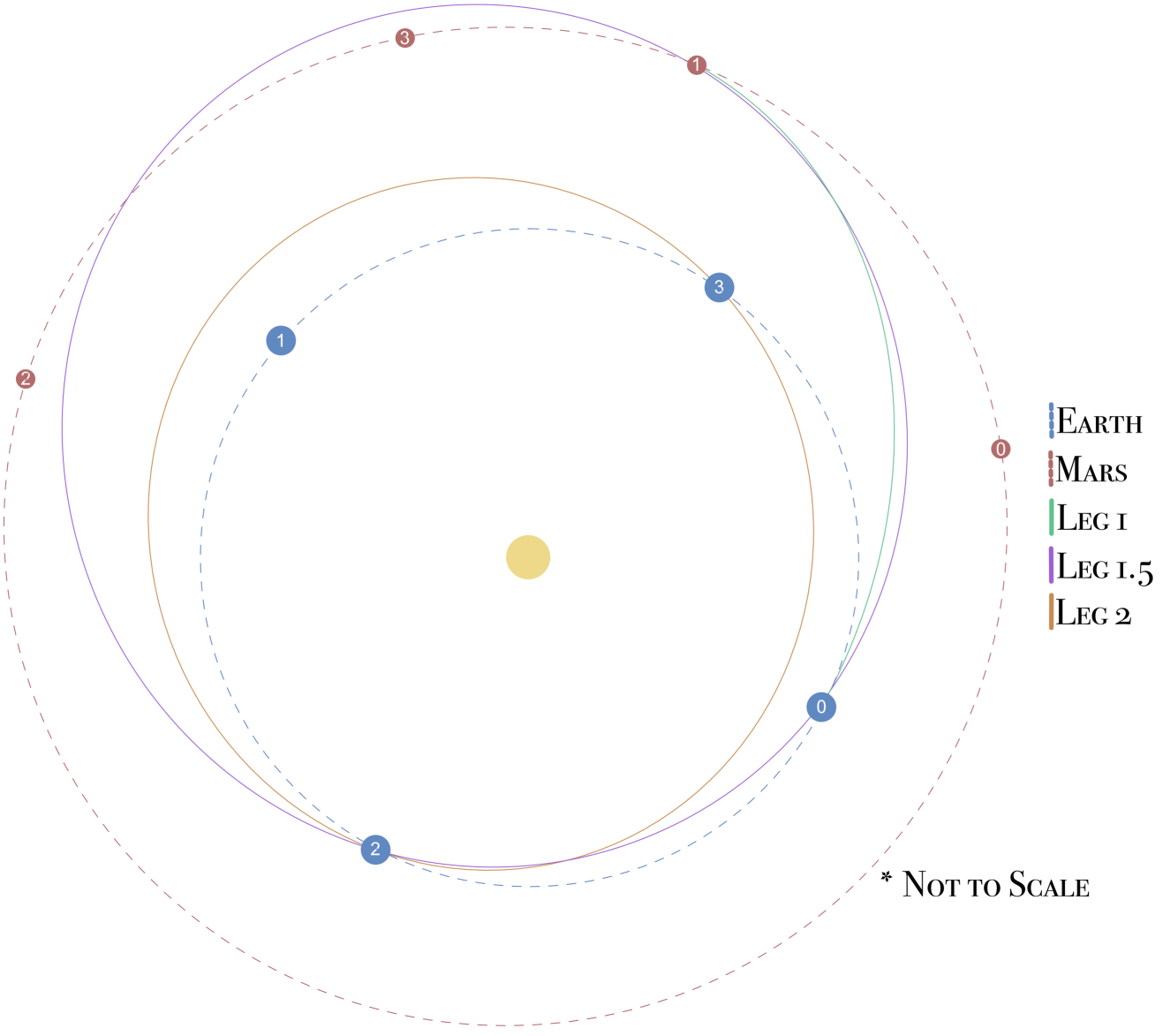

Figure 2.5: The Ballistic S1L1 Outbound Cycler first two legs (Ephemeris Model) 
Chapter 3

\section{MANIFOLD DYNAMICS EXAMINATION}

This chapter will briefly discuss the background and of the dynamics of manifolds as well as the 3-body dynamics required to access the manifold solutions necessary for this work. The concepts required to evaluate these solutions will also be analyzed.

\subsection{Background of Manifold Dynamics}

For most, if not all of spaceflight history, the trajectories of spacecraft have been determined through the use of patched conics and planetary flybys. Despite the usefulness and rigidness of these 2-body methods, they can require precise timing, a relatively large amount of fuel consumption, and can be inflexible in handling more complicated mission requirements. In order to allow for more complicated mission trajectories and requirements, the dynamics of multi-body systems can be utilized. The concepts involved with multi-body dynamics and manifolds is very complicated and will be discussed in detail here.

The dynamics of multi-body systems, and 3-body systems in particular, stem from the circular restricted 3-body problem, which describes the motion of an object around 2 other large bodies. Though this problem has been known for around 200 years, the

formulation of the equations of motion were not well-described until 1967[20], and one of the first missions to utilize them was the ISEE-3 which didn't launch until $1978[6]$.

One of the most notable missions to date that will make use of these dynamics is the James Webb Space Telescope (JWST)[12]. Through the utilization of halo orbits around the Earth-Sun L2 point and corresponding approach trajectories on a 
manifold, the telescope is capable of communicating with Earth, unimpeded, and can arrive with very low $\Delta V$ cost with respect to the distance travelled. The fundamentals of this mission are helpful in understanding halo orbit insertion, which will be discussed later.

\subsection{The Circular Restricted 3-Body Problem}

Before the dynamics of manifold trajectories are discussed in any form, it is necessary to introduce the underlying physics of the matter. The circular restricted 3-body problem (CR3BP) describes the effects that two separate large bodies, dubbed the primary and secondary, have on a much smaller third body, called the tertiary body[3]. As a side note, some call the larger bodies the smaller and larger primary, but for our purposes, we will use the previous nomenclature. The geometry used to represent this problem is given in Fig. 3.1. It is important to note that for most systems, the barycenter of the two objects will be contained within, and usually very close to the center of, the primary body.

The dynamics of the CR3BP primarily take place in what is called the fixed frame, or sometimes called the synodic frame. This frame does not move with respect to the secondary and allows the trajectory with respect to the secondary become more apparent. It also allows the equations of motion to greatly simplify. For the most part, trajectories under the influence of CR3BP dynamics will be plotted in this frame.

In order to make the dynamics of this problem a little more manageable, the equations are derived using a unit system known as canonical units. These are units uniquely defined by a parameter in the system that we want to normalize, such as the

distance between two objects for example. For the purposes of CR3BP, the masses of the primary and secondary bodies in the system are used to define the desired 


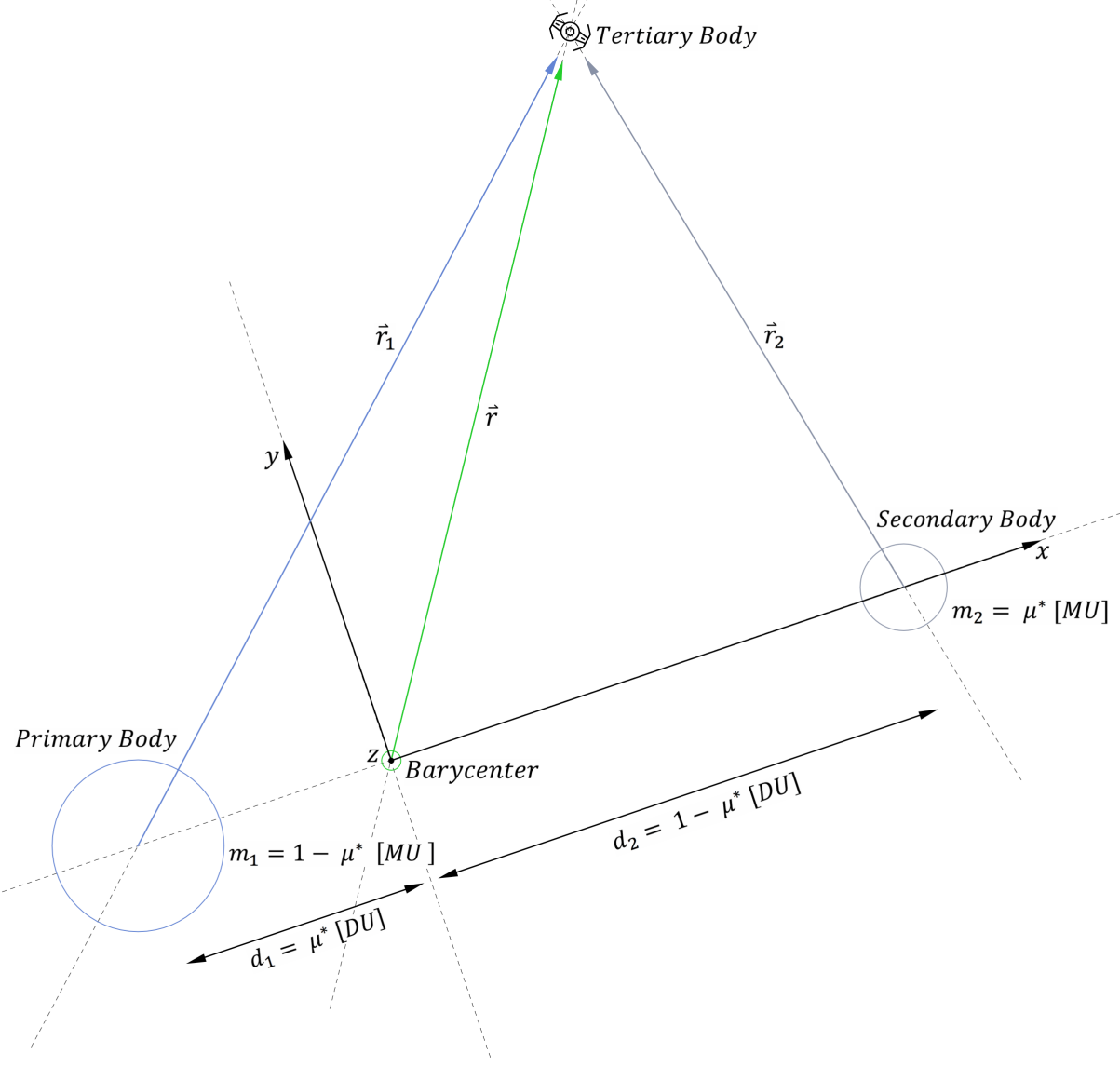

Figure 3.1: Circular Restricted 3-Body Problem Geometry 
canonical units. The parameter in question is given through the following equation:

$$
\mu^{*}=\frac{m_{2}}{m_{1}+m_{2}}
$$

where $m_{1}$ and $m_{2}$ are the masses of the primary and secondary bodies respectively.

The assumptions necessary for these dynamics to work are listed as follows:

- All bodies will be modeled as point masses

- There are no orbital perturbations present (gravity is only acting force)

- The primary and secondary bodies have circular orbits around the barycenter of the system

- $m_{3} \lll m_{2}<m_{1}$

The diagram in Fig. 3.1 gives an idea of how masses and distances can be defined in terms of mass units $[\mathrm{MU}]$ and distance units [DU]. After normalizing the system with $\mu^{*}$, the properties of the system are as follows.

$$
\begin{aligned}
m_{1}+m_{2}=1 & {[\mathrm{MU}] } \\
d_{1}+d_{2}=1 & {[\mathrm{DU}] }
\end{aligned}
$$

where, $1 \mathrm{DU}$ is equivalent to the distance between the primary and secondary in $\mathrm{km}$, and $1 \mathrm{MU}$ is equivalent to the mass of the entire system in $\mathrm{kg}$. Furthermore, the derived units are given below.

$$
\begin{aligned}
& {[\mathrm{TU}]=\sqrt{D U^{3} / \mu}} \\
& {[\mathrm{VU}]=D U / T U}
\end{aligned}
$$


where $\mu$ is the standard gravitational parameter of the primary body in $\mathrm{km}^{3} \mathrm{~s}^{-2}$. It is also important to note that the barycenter of the system is fixed to be at the point $(0,0)$ in the $x-y$ plane.

To derive the equations of motion, the gravitational effects of the objects are modeled as point forces. A detailed derivation can be found in reference [3]. Summing the forces over all directions gives the neatly summarized accelerations as

$$
\begin{aligned}
& \ddot{x}=2 \dot{y}+x-\left(1-\mu^{*}\right) \frac{x+\mu^{*}}{r_{1}^{3}}-\mu^{*} \frac{x-1-\mu^{*}}{r_{2}^{3}} \\
& \ddot{y}=-2 \dot{x}+y-\left(1-\mu^{*}\right) \frac{y}{r_{1}^{3}}-\mu^{*} \frac{y}{r_{2}^{3}} \\
& \ddot{z}=-\left(1-\mu^{*}\right) \frac{z}{r_{1}^{3}}-\mu^{*} \frac{z}{r_{2}^{3}}
\end{aligned}
$$

where,

$$
\begin{aligned}
& r_{1}=\sqrt{\left(x+\mu^{*}\right)^{2}+y^{2}+z^{2}} \\
& r_{2}=\sqrt{\left(x-1+\mu^{*}\right)^{2}+y^{2}+z^{2}}
\end{aligned}
$$

Due to the nature of these equations, there exist no analytic methods of integrating them over time. To mitigate this, many numerical methods exist to describe the motion of an object under these dynamical laws. For the sake of conciseness, such a method will be observed when discussing the computation of halo orbits. It is also very important to note that all equations of motion when dealing with the CR3BP are in the synodic frame, which means that the position of the planet will be fixed according to the equations of motion.

\subsubsection{Libration Points}

One of the underlying and most prevalent effects of CR3BP are the gravitational equilibrium points, termed Lagrange points or libration points. In each system, there exists 5 libration points where no acceleration of a third body (assumed to lie in 


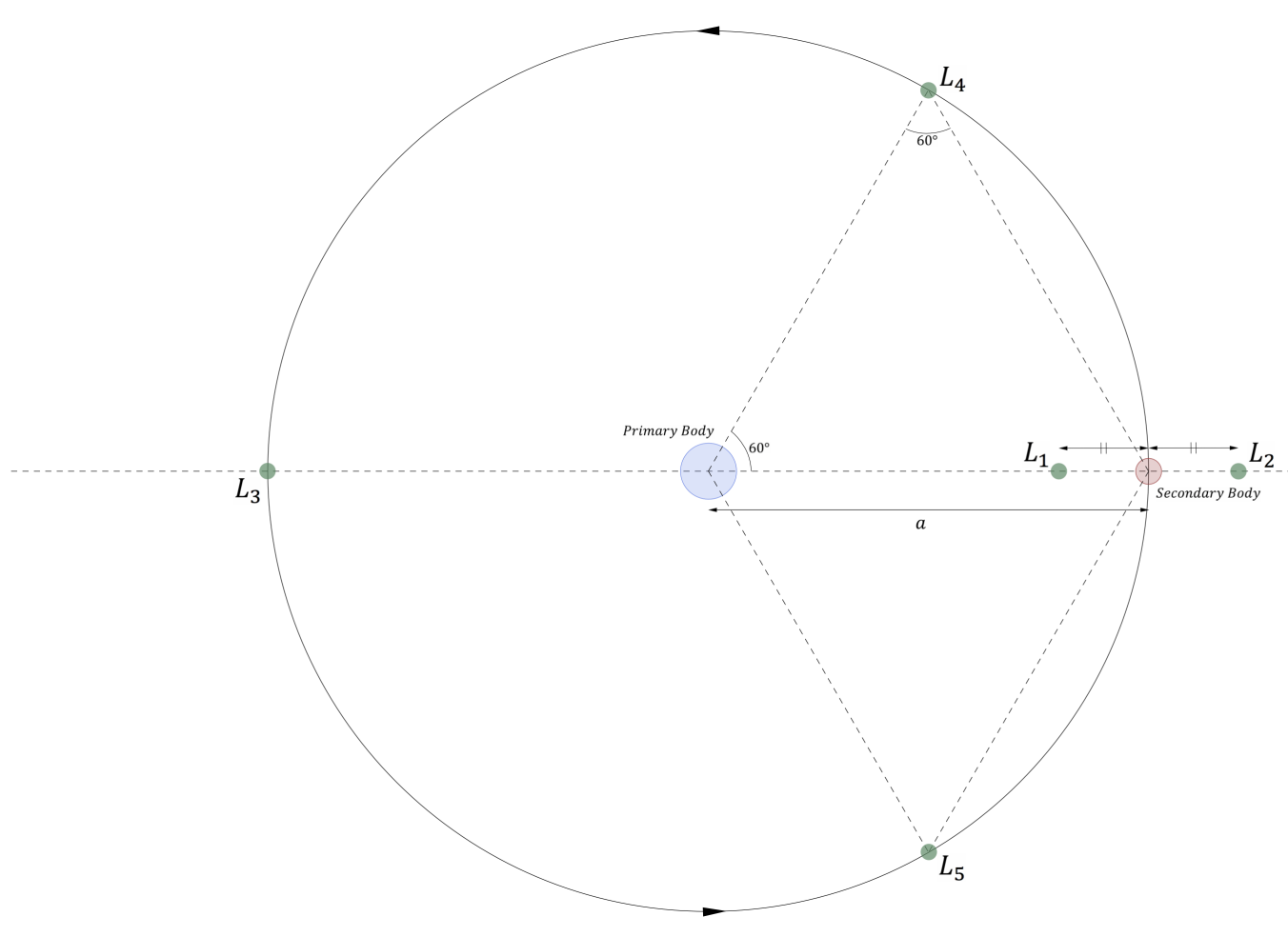

Figure 3.2: Libration point locations for generic system

the $\mathrm{x}-\mathrm{y}$ plane) occurs. The positions of these points can be found by setting the accelerations in $\mathrm{x}$ and $\mathrm{y}$ to zero, as well as any velocities. This gives the following:

$$
\begin{aligned}
x-\left(1-\mu^{*}\right) \frac{x+\mu^{*}}{r_{1}^{3}}-\mu^{*} \frac{x-1-\mu^{*}}{r_{2}^{3}} & =0 \\
y-\left(1-\mu^{*}\right) \frac{y}{r_{1}^{3}}-\mu^{*} \frac{y}{r_{2}^{3}} & =0
\end{aligned}
$$

In order to solve for all solutions to $\mathrm{x}$ and $\mathrm{y}$, one must solve a quintic equation. This gives the 3 co-linear points, L1, L2, \& L3, and the 2 triangular points, L4 \& L5. In general, L1 lies between the primary and secondary, L2 lies beyond the secondary, L3 is on the far side of the primary, L4 lies at an upper equilateral triangle to the primary and secondary, and similarly L5 lies at the lower triangle. A diagram for a generic system of libration points is given in Fig. 3.2.

For reference in a physical application, if a point mass were to be placed at any of these libration points with no initial velocity, it would remain in that exact spot 
relative to the primary and secondary body. These points can also be orbited in a special trajectory called a halo orbit - sometimes called a periodic orbit around a libration point - and can even be approached/departed in a sort of free ride known as a manifold trajectory. It also is interesting to mention that the co-linear points are unstable and slight perturbations can effect the position of the point mass heavily at the points. However, this instability will not be considered for the purposes of this work.

\subsection{Halo Orbits}

As previously mentioned, halo orbits are perfectly periodic trajectories about a libration point, similar to a traditional orbit around a body. There are a few key differences however, that make these trajectories extremely unique. These are also not the only orbital paths around libration points; which includes lissajous and Lyapunov orbits, but these will not be discussed here. In order to spare an ample amount of derivation, the computation of these trajectories will not be presented here. However, a detailed method for their computation can be found in a paper written by Megan Rund[17]. The calculation begins with an approximate, analytic solution, which is then iterated upon through a numerical error method. This mechanism provides a sufficiently adequate solution of the halo orbit and has been deemed suitable for the purposes of this work.

Halo orbits do not form a traditional orbital shape - such as an ellipse - and instead display a saddle geometry that curves around the libration point. There are two main factors that determine the shape of the halo orbit: first is its out-of-plane amplitude, hereafter referred to as $z$-Amplitude or $A_{z}$; the next is its class, either northern or southern, which determines the orientation of its curvature around the libration point. The classes are related through the symmetry $z \rightarrow-z$ and are purely 

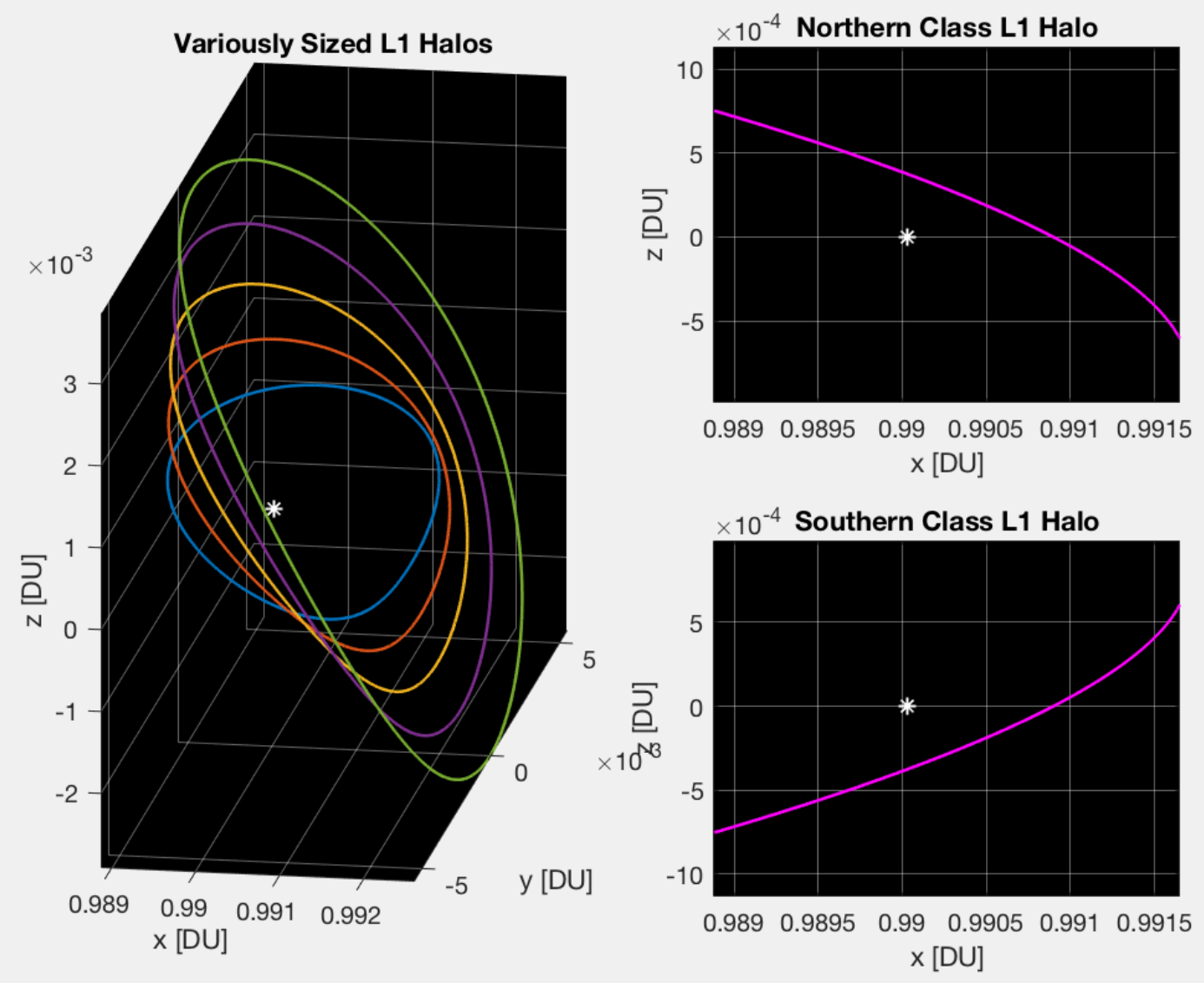

Figure 3.3: Various halo orbits about Earth-Sun L1

a consequence of symmetry[10]. An example of various halo orbit shapes and sizes can be found in Fig. 3.3.

\subsection{Manifolds}

Due to the dynamics of the CR3BP, there are a few interesting anomalies that can occur; namely with the trajectories that spacecraft can take. Manifolds are a collection of trajectories that approach or depart a particular libration point or libration orbit. Any object that can somehow maneuver onto a trajectory in a manifold will continue to follow this trajectory until libration point/orbit insertion, or until the influence of the CR3BP is no longer active. Due to the nature of the system, the shape of these 
manifolds can vary widely.

Before discussing shape, however, it is necessary to discuss the various categories of manifolds. Manifolds can belong the the stable or unstable denomination which denotes whether the trajectory approaches or departs a libration orbit (or libration point) respectively. In many cases, the stable manifold is the most explored since the libration orbit itself is the desired result. However, the unstable manifold can be used to send a spacecraft beyond the system in question. The unstable manifold will prove critical to the success of this thesis later on.

The invariance of manifolds is also an important factor to be considered. Invariant manifolds are those that will always approach or depart a halo orbit, while the variant variety cannot be mapped to a periodic orbit around a libration point[16]. For the purposes of this work, only the invariant manifolds will be used. To visualize the shape of the invariant manifolds, a plot of the stable and unstable variety from an L1 halo orbit are given in Fig. 3.4. It is clear from the diagram that far from the halo orbit, the trajectories on the manifold follow a semi-regular pattern and form a tube shape that is common to most manifolds. However, very near the halo orbit, the dynamics are very complicated and do not form any recognizable shape. This should not prove to be an issue however, as the dynamics in this region are predictable.

Since the manifold is a collection of trajectories, only a single trajectory within the manifold can be chosen for a specific mission purpose. A method for choosing one of these trajectories, relevant to this work, will be discussed in great detail in Chapter 5. 

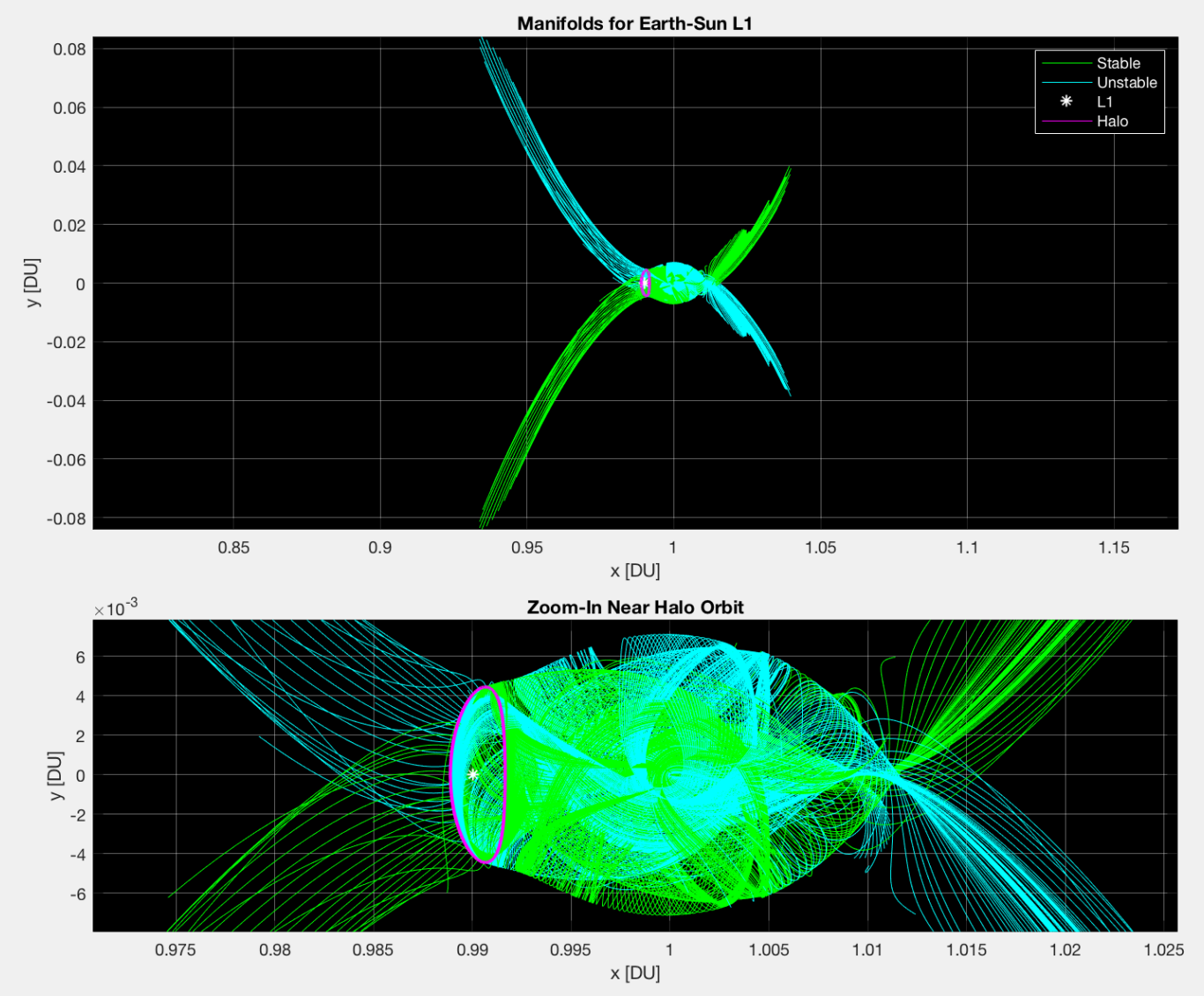

Figure 3.4: Manifolds approaching and departing L1 halo 
Chapter 4

\section{CYCLERS}

The cycler trajectory can be a complicated path to understand, and an even more complicated one to find; that is, in the Ephemeris model of the solar system. One of the most pressing questions that one may have is why the cycler trajectory is calculated in the ephemeris model, when the manifolds have to be calculated using a circular model of the solar system.

Although the CR3BP obviously requires the assumption of a circular orbit of the secondary body, the dynamics of the CR3BP have proven to be adaptable to the accurate model of the solar system. Another point, is that the circular solution of the cycler trajectory simply can’t be used for practical application; as was discovered by McConaghy et al.[13], when adapting a cycler trajectory to the ephemeris model, the circular solution cannot even be used as an initial guess for the solution as the algorithm can never converge. This de-legitimizes the circular solution as a valid feasibility study.

Because the goal of this work is to prove feasibility of the manifold-to-cycler system, it is necessary to obtain the most reasonably accurate model in order to determine a valid solution space. With that in mind, this chapter will discuss the fundamentals of obtaining a cycler trajectory in the ephemeris model of the solar system.

\subsection{Verifying The Ephemeris S1L1-B Cycler}

Before discussing the method developed for generating the cycler trajectory, a method for verifying existing cycler trajectories will be presented here. This is because the 
underlying concepts of a cycler's verification feed into its generation; a fact that will become clear later. In order to demonstrate this process, the itinerary presented in Chapter 2 in Table 2.1 will be used.

\subsubsection{Pork-Chop Plots}

The principle method for which verification will occur relies on an extension to the pork-chop plot. These are plots that demonstrate the $V_{\infty}$ (hyperbolic excess velocity) contours plotted against the launch and arrival dates for an interplanetary flight. Sometimes, instead of $V_{\infty}$ the characteristic energy $\left(C_{3}\right)$ is used instead. As a visual example, Fig. 4.1 provides a pork-chop plot for the first leg of the particular cycler of study; that being the transfer from Earth to Mars. Since the launch and arrival dates are known, I extended the search space to include 13 days before and after each of these dates in order to generate a plot with more than one solution.

Notice that the $V_{\infty}$ at both Earth and Mars are considered, as well as the time of flight. This allows for anyone designing a mission profile to quickly choose the transfer that is best for them simply by looking for the most desired intersection of the contours. For the purposes of this work however, it is necessary to generate 2 pork chop plots for any $1 \mathrm{Leg}$ in order to guarantee that the flyby is ballistic. After the spacecraft travels from Earth to Mars, it must travel back to Earth using only a ballistic flyby, so another plot is generated to show the possibilities of the flight back; which is given in Fig. 4.2.

Since the $V_{\infty}$ contours at Mars are presented in both charts, one can simply find where they are the same and this is where the possibility of a ballistic flyby occurs. It is important to keep in mind that the arrival date of Mars must be the same as its departure date when determining this flyby. The same process can be carried out for the Earth-Earth transfer that occurs afterwards. 


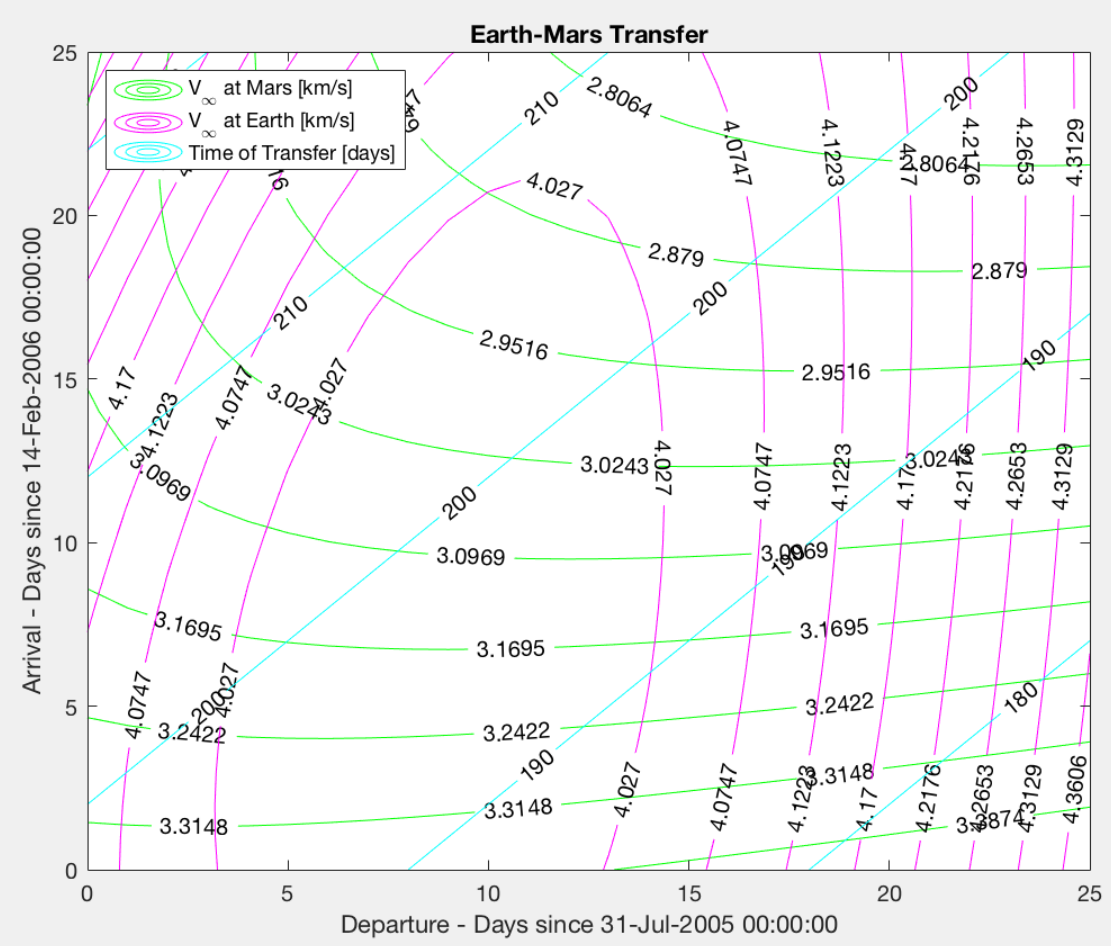

Figure 4.1: Pork-chop plot for Earth-Mars transfer

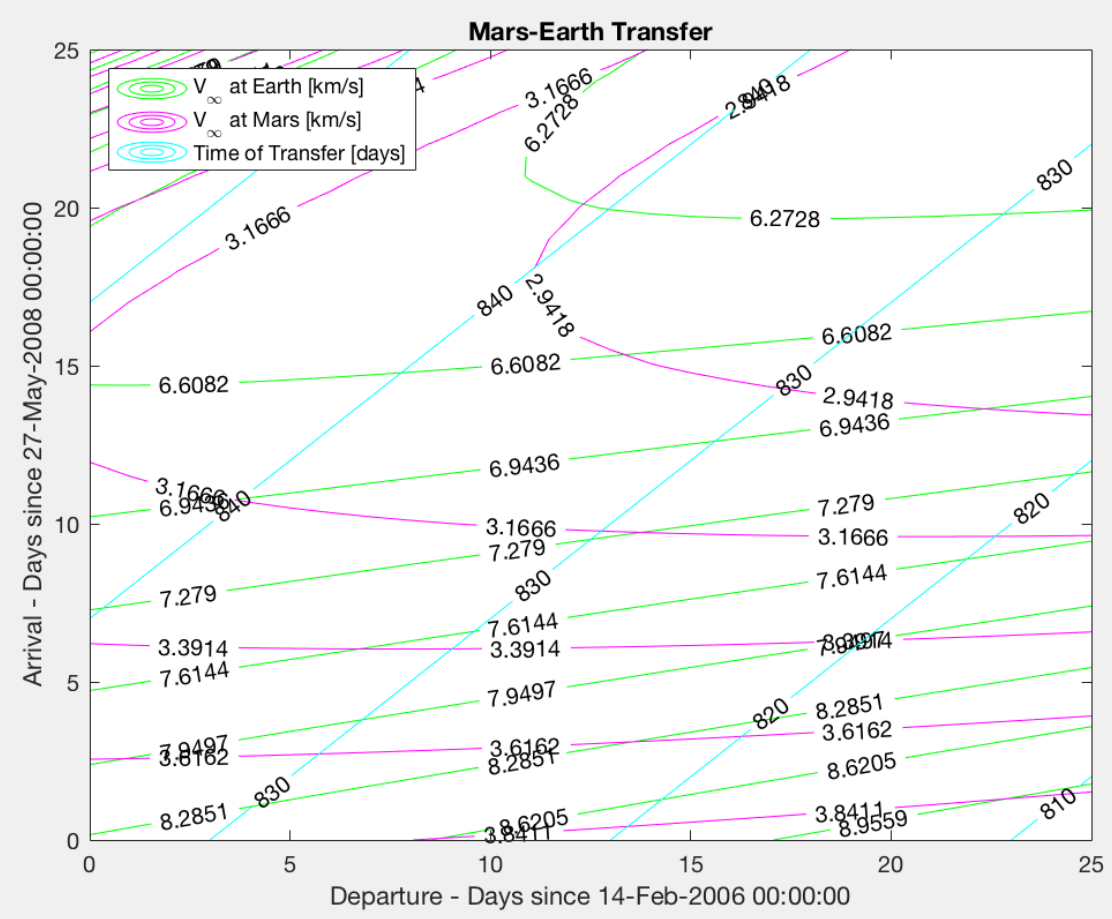

Figure 4.2: Pork-chop plot for Mars-Earth transfer 


\section{$V_{\infty}$ Surface Plots}

Unfortunately, the previous process is relatively under-detailed and only truly allows for a visual marker into the actual flyby that is needed. Scouring various methods for a way to accomplish this more favorably proved fruitless, thus it was decided that developing a new method for determining the flyby date (launch and arrival dates) was necessary. This method works by consolidating the pork-chop plots into a surface map that only shows the possible ballistic flyby dates; this will be hereafter referred to as a $V_{\infty}$ surface plot.

The method for generating the pork-chop plots already obtains matrices of all possible $V_{\infty}$ values for approaching and departing a planet (depending on departure and arrival dates), these values can be consolidated into two surface plots and overlayed graphically. The effect of this graphing method allows the intersection contour of the two surface plots to be visualized as well as directly calculated through mesh intersection algorithms. An example of a surface plot generated using the previously shown pork-chop plots is given in Fig. 4.3.

The approaching and departing surfaces contain all possible values of the $V_{\infty}$ based the dates at which they occur. In this case, for the approaching plane, the departure dates of Earth and the arrival dates of Mars are considered, whereas with the departing plane, the departure dates from Mars and the arrival dates at Earth are considered. The importance here is that the arrival and departure dates of Mars should be the same (or at least within 24 hours to account for the flyby time); this allows the two surfaces to be plotted atop one another. The intersection of these planes, the magenta dashed line, gives the corresponding dates at which a ballistic

flyby is possible. These intersections or contours will prove important when discussing the cycler timeline. 


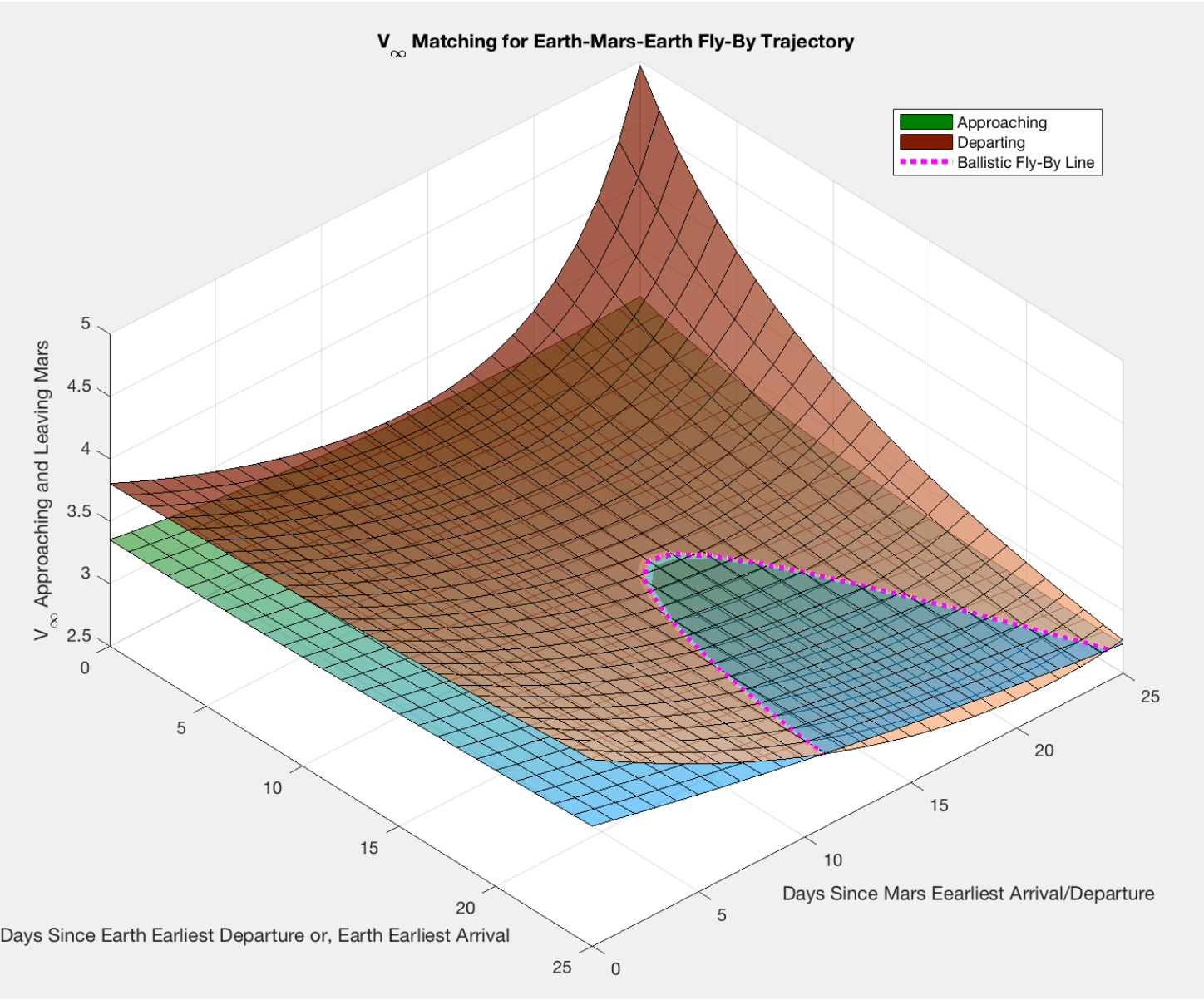

Figure 4.3: $V_{\infty}$ surface plot for Earth-Mars-Earth trajectory 


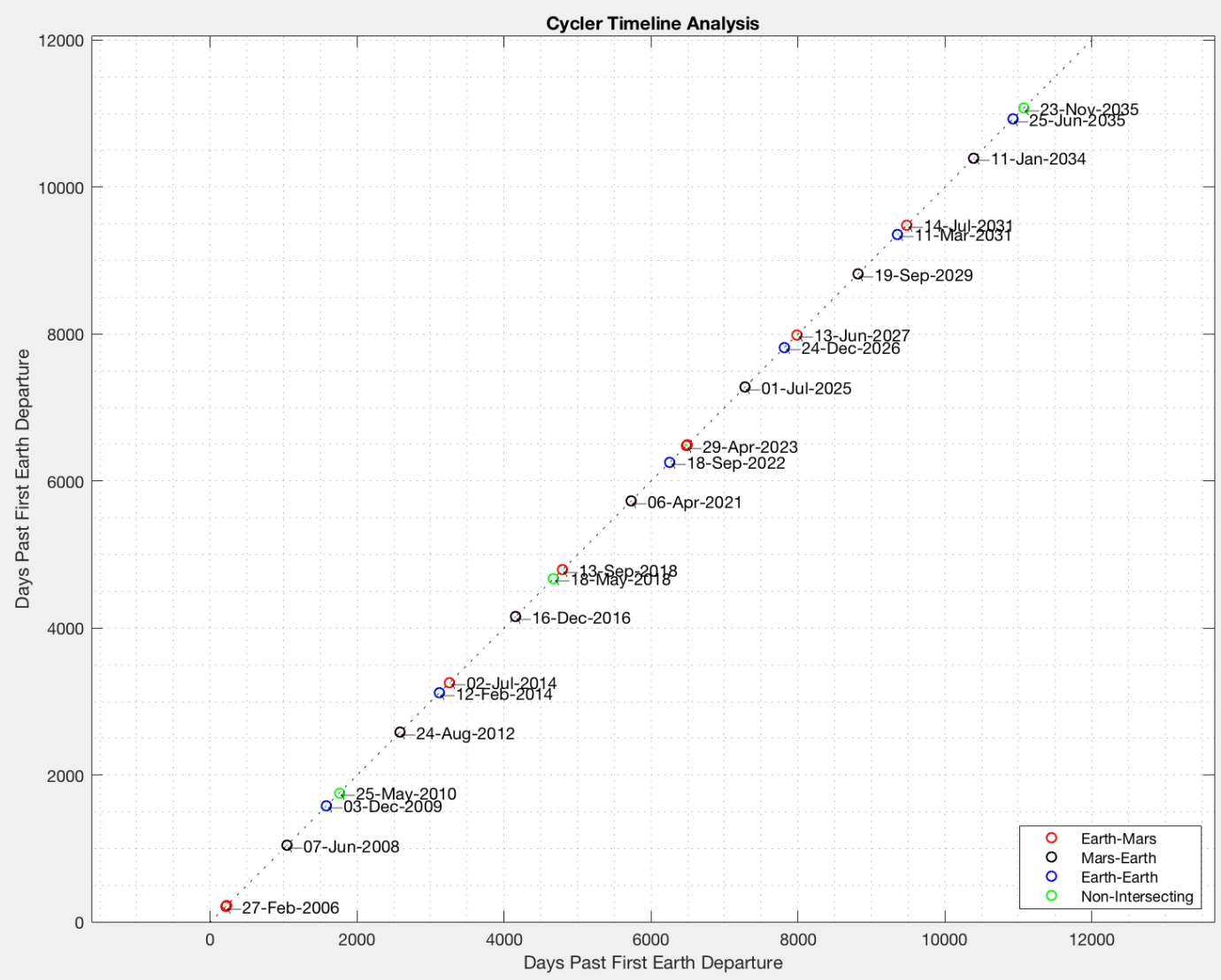

Figure 4.4: Generated timeline from known itinerary

\section{Timeline}

By generating a plot of this sort for every flyby in the cycler itinerary, all ballistic flyby contours can be generated for the cycler trajectory. Each one can then be laid on an $\mathrm{x}-\mathrm{y}$ coordinate frame where each axis represents time in days past earliest launch from Earth. A line of $x=y$ can then be drawn through all of the contours, termed the timeline from now forward. Wherever this timeline intersects with the contour, that is the date at which a ballistic flyby should occur. The goal is the make sure that the timeline crosses the path of every single contour in the the $\mathrm{x}-\mathrm{y}$ time-plane we have created. To illustrate this, a plot of this process can be found in Fig. 4.4.

Some will notice that there are some contours that do not intersect the timeline 
- labeled as "Non-Intersecting" in the figure. This is due to the fact that only entire days are considered and the time it takes to complete a flyby is not. This causes some curves to come very close to, but not directly cross the timeline. To mitigate this, the closest point on the contour to the timeline is chosen to act as the flyby date. For the interested, an illustrative plot of some of the intersections of contours and the timeline can be found in Appendix A.

When comparing the found flyby dates to the original itinerary, found in Table 2.1, one can see that they are quite similar, with some admitted variation. This is mostly attributed to the fact that the itinerary was originally generated through an optimization, whereas this replica was generated through flyby contours. However, both are technically valid and it is now possible to extend this method of verification to generating an entirely new cycler trajectory, albeit of the same shape.

\subsection{Cycler Generation}

Though not entirely the focus of this work, in order to prove the feasibility of cycler insertion via manifolds, it is necessary to first prove that a proper cycler trajectory can be generated in the first place. Though there have been a few works illustrating this possibility, their methods were unadaptable for this work as powerful optimization software was used and the original authors of the method had not worked on the

problem in over a decade[15]. It was also found that cyclers can be generated via an adaptation of the previously derived verification method.

\subsubsection{Transfer Times}

The method designed for this work involves using known cycler itineraries to obtain a general time line to search around. This is down by finding the average transfer times between each of the three transfers, those being Earth-Mars, Mars-Earth, and Earth- 


\section{Table 4.1: Transfer times for inbound and outbound cyclers}

\begin{tabular}{ccc} 
Trajectory & Outbound [days] & Inbound [days] \\
\hline Earth-Mars & $161 \mid(115,231)$ & $868 \mid(788,938)$ \\
Mars-Earth & $862 \mid(788,930)$ & $159 \mid(108,225)$ \\
Earth-Earth & $536 \mid(529,542)$ & $536 \mid(529,542)$
\end{tabular}

Earth. Using the itineraries provided by T. McConaghy[15], the average transfer times were determined for the outbound and inbound cyclers, which can be found in Table 4.1.

There are a few interesting things of note here. Firstly, the Earth-Mars outbound trajectory has very similar characteristics to the Mars-Earth inbound trajectory. The same relationship is true for Mars-Earth outbound vs. Earth-Mars inbound. This makes sense due to the fact that although the trajectories have the same shape, they are designed for the exact opposite purpose. Notably, the Earth-Earth trajectories not only have similar characteristics between inbound and outbound trajectories, but they are the exact same. Furthermore, their transfer time range is much tighter than the other transfers, with a maximum range of only 13 days.

\subsubsection{Relative Positions}

The ranges of these transfer times are now used as the basis for how broad a search must be performed by the $V_{\infty}$ surface plots, with a bit of leeway for outliers that may occur. It is always possible to start the search from any launch and arrival dates, but these must be chosen wisely in order to insure that this method converges to a solution quickly. Because the first Earth-Mars transfer has to maintain a certain shape, the relative positions of Earth and Mars at the launch date are relatively constrained. That is to say that in order to exclude solutions from the space that have unorthodox transfer times, the relative angular positions of Earth and Mars must be maintained 
Table 4.2: Relative angular positions of Earth and Mars

\begin{tabular}{c|cc}
\hline & Radians [rad] & Degrees [o] \\
\hline Outbound (Earth-Mars) & $4.5392 \mid(4.3309,4.7507)$ & $260.08 \mid(248.14,272.20)$ \\
Inbound (Mars-Earth) & $3.5866 \mid(3.4007,3.6737)$ & $205.50 \mid(194.84,210.49)$ \\
\hline
\end{tabular}

within a certain range. To find this range, a similar analysis to the previous was performed but instead found the average relative angular position of Earth to Mars just before the Earth-Mars transfer. This does not give insight into the complete cycler, but only the possible launch dates from Earth at the start of any given cycler.

The relative angular positions of Earth and Mars can be found through their angular offset from a chosen reference direction, $\Upsilon$, with direction $[1,0,0]$ relative to the sun. The angular position of a planet, $\phi_{p}$ can be found via the following:

$$
\phi_{p}=\omega+\theta-2 \pi m
$$

where, $\omega$ is the argument of periapse of the orbit and $\theta$ is the true anomaly of the current planet position. Also, $m=0,1$ and the entire term is meant to wrap the angle, $\phi_{p}$ to $[0,2 \pi)$. Using Eq. 4.1, the relative angular positions of Earth and Mars, $\phi$, can be found at any time by simply subtracting to two. As a quick note, Earth's angular position is always subtracted from Mars', so a positive angle represents Mars ahead of Earth while a negative represents the opposite. A summary of the average relative angles for Earth-Mars outbound and Mars-Earth inbound trajectories are given in Table 4.2 .

\subsubsection{The Search}

We now have all of the data and tools necessary to begin searching for and computing S1L1-B cycler trajectories of the inbound and outbound classes. Though this is most certainly a brute force method for calculating a cycler trajectory, most of the 
complications that come along with an optimization are remedied. As a quick note, the flyby altitude, $r_{p}$, for each flyby will not be calculated until the entire cycler trajectory is generated. This is mostly due to the fact that the flyby can occur at any of the possible times (which will have varying $r_{p}$ and these times cannot be completely determined until the entire trajectory is known.

To get a general idea of how these itineraries are generated, the following is a list of steps taken to determine them.

1. Choose an arbitrary launch date from Earth

2. Calculate the relative angular position, $\phi$, to Mars and adjust launch date until within known bounds (Table 4.2)

3. Using the known transfer time bounds (Table 4.1) for the desired Earth-Mars trajectory, generate a $V_{\infty}$ surface plot for all possible arrival and departure dates around the chosen launch date

4. Choose an arrival date from the generated possibilities and repeat the process for a Mars-Earth transfer, then for an Earth-Earth and so on

5. Repeat until no possible arrival/departure dates can be found and adjust the initial arrival date at Mars

6. Repeat entire process until a feasible itinerary is found; generating a timeline and looking for intersections

7. Calculate all flyby altitudes, $r_{p}$, and $v_{\infty}$ s for the trajectory

8. If a flyby crashes into a planet, start the process over entirely

One may see this as an extremely arduous and painstaking process. However, this method is only meant to generate a few cycler trajectories to prove the concept as 
well as form a basis for the rest of the work. Because most of the visualization of the process is contained within the previous sections of this chapter, that will not be presented here, however, a few steps of the process and a few extra itineraries can be found in Appendix A.

However, to demonstrate that this process does work, an itinerary and visual representation of an outbound cycler in Table 4.3 \& Fig. 4.5 as well as an inbound cycler in Table 4.4 \& Fig. 4.6 are provided. Notice that only given the first 3 legs are given in the visual representation; this is due to the fact that although there are slight variations in the shape of each similar leg, plotting every leg would not be useful in understanding the repetition of the cycler trajectory. Furthermore, the third leg is sufficient in demonstrating this repetition since it looks similar in shape and scope to the first leg.

As a quick note, it is necessary to mention that a visual representation of any of the flybys will not be presented here. This is because the flybys are modeled as instantaneous rotations of the velocity vector and will therefore not take the shape of the flyby diagram, Fig. 2.1 in Chapter 4. However, this diagram should be satisfactory in demonstrating the shape that a flyby trajectory takes.

\subsection{Insertion $\Delta V$}

With that being said, we now have cycler trajectories ready to be inserted onto with the help of manifolds. It is important to note that only the first leg of each cycler is necessary for insertion since the cycler is self-repeating. However the first leg of a cycler had to be found before it could be utilized in any appropriate manner. Before moving on to manifolds however, a quick number will be generated for comparison purposes later. Since the interest in this mission lies with the possibility of reducing the total $\Delta V$ required, it is first vital to know what a normal burn would require. 
Table 4.3: Generated outbound cycler trajectory itinerary

\begin{tabular}{|c|c|c|c|c|c|c|c|}
\hline \multicolumn{8}{|c|}{ Outbound Cycler Vehicle 1} \\
\hline Encounter & Fly-By Planet & Year & Month & Day & $V_{\infty}$ & $\begin{array}{l}\text { Closest } \\
\text { Approach } \\
\text { Distance }\end{array}$ & Leg, TOF \\
\hline$\#$ & $P$ & yyyy & $\mathrm{mm}$ & $d d$ & $\mathrm{~km} / \mathrm{s}$ & $k m$ & days \\
\hline 1 & Earth & 2007 & 9 & 30 & 4.52 & -- & -- \\
\hline 2 & Mars & 2008 & 5 & 19 & 3 & 6600 & 231 \\
\hline 3 & Earth & 2010 & 7 & 16 & 7.05 & 25000 & 788 \\
\hline 4 & Earth & 2012 & 1 & 9 & 7.06 & 37500 & 542 \\
\hline 5 & Mars & 2012 & 6 & 17 & 5.89 & 9800 & 160 \\
\hline 6 & Earth & 2014 & 10 & 11 & 5.33 & 25500 & 846 \\
\hline 7 & Earth & 2016 & 3 & 29 & 5.31 & 35200 & 535 \\
\hline 8 & Mars & 2016 & 7 & 25 & 7.87 & 9600 & 118 \\
\hline 9 & Earth & 2019 & 2 & 4 & 3.99 & 22800 & 924 \\
\hline 10 & Earth & 2020 & 7 & 17 & 3.98 & 4270 & 530 \\
\hline 11 & Mars & 2020 & 12 & 18 & 4.36 & 5150 & 154 \\
\hline 12 & Earth & 2023 & 5 & 25 & 6.08 & 20050 & 887 \\
\hline 13 & Earth & 2024 & 11 & 13 & 6.1 & 27300 & 538 \\
\hline 14 & Mars & 2025 & 6 & 1 & 3.71 & 16100 & 200 \\
\hline 15 & Earth & 2027 & 8 & 8 & 6.72 & 25900 & 799 \\
\hline 16 & Earth & 2029 & 1 & 30 & 6.73 & 41300 & 541 \\
\hline 17 & Mars & 2029 & 6 & 27 & 6.62 & 16700 & 148 \\
\hline 18 & Earth & 2031 & 11 & 14 & 446 & 32000 & 871 \\
\hline 19 & Earth & 2033 & 4 & 29 & 4.47 & 24900 & 532 \\
\hline 20 & Mars & 2033 & 8 & 18 & 7.58 & 7100 & 111 \\
\hline 21 & Earth & 2036 & 3 & 6 & 4.72 & 22300 & 930 \\
\hline 22 & Earth & 2037 & 8 & 20 & 4.74 & 600 & 532 \\
\hline 23 & Mars & 2038 & 1 & 22 & 5.66 & 1450 & 155 \\
\hline 24 & Earth & 2040 & 5 & 11 & 11.05 & -- & 840 \\
\hline
\end{tabular}



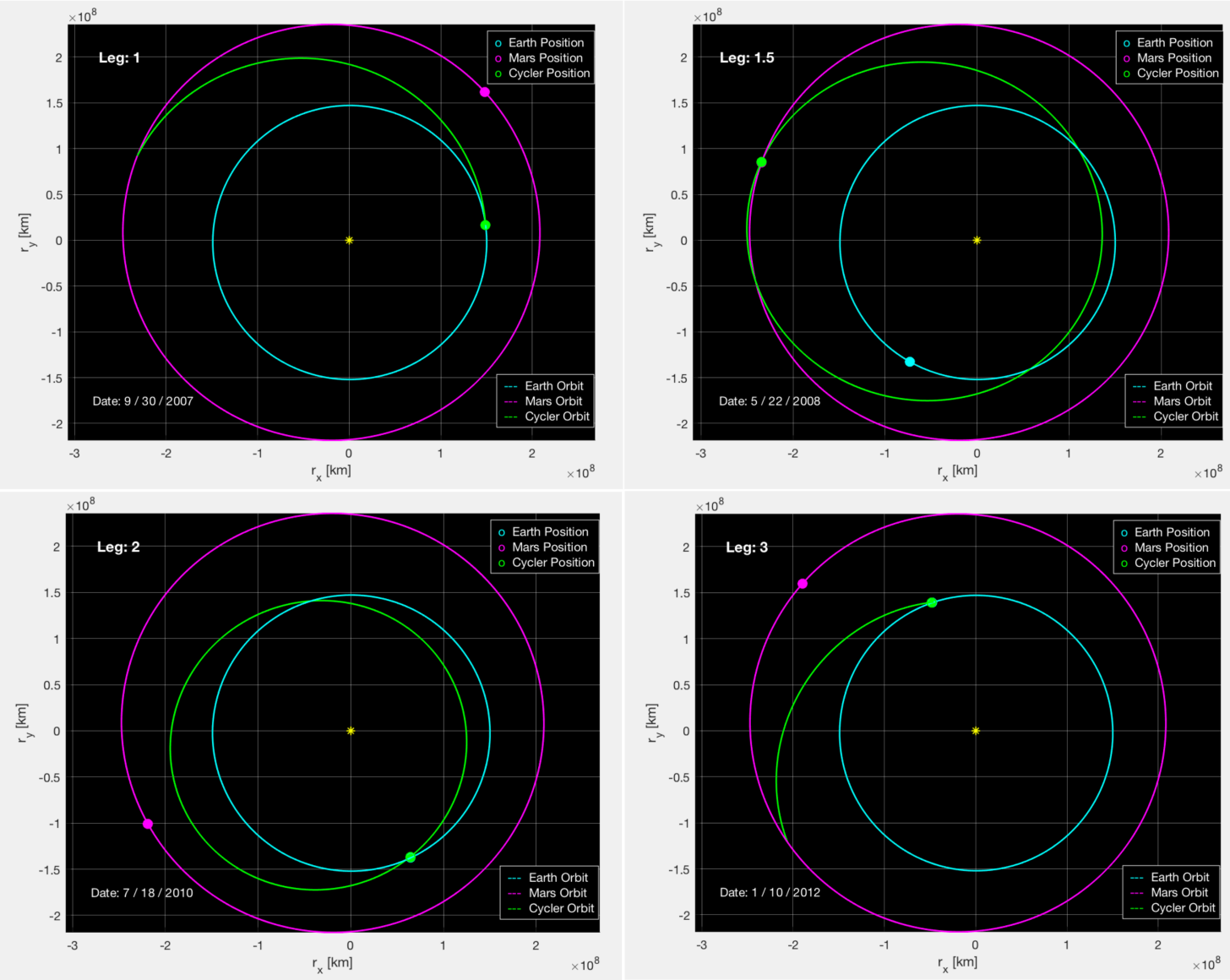

Figure 4.5: First 3 legs of generated outbound cycler 
Table 4.4: Generated inbound cycler trajectory itinerary

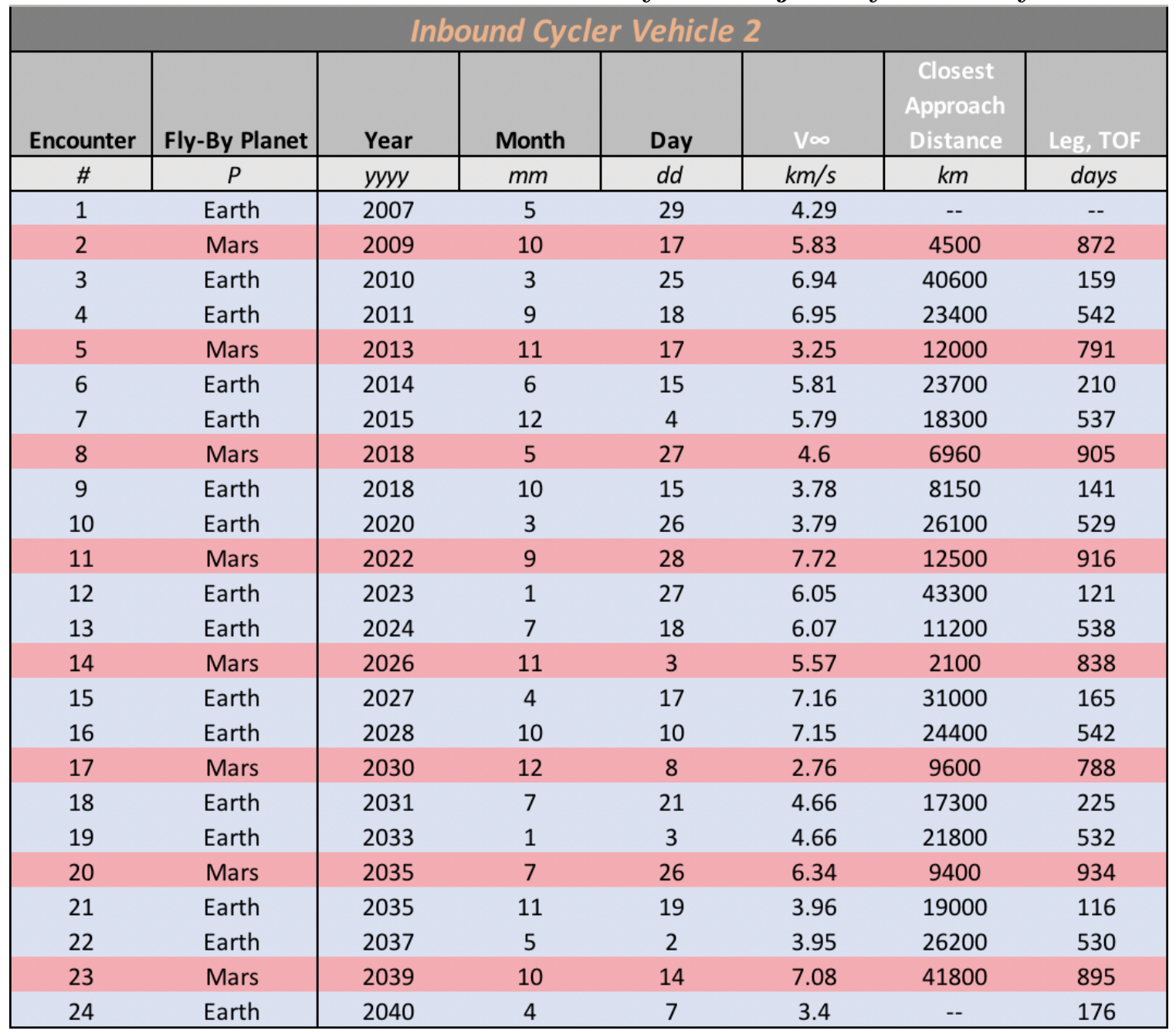



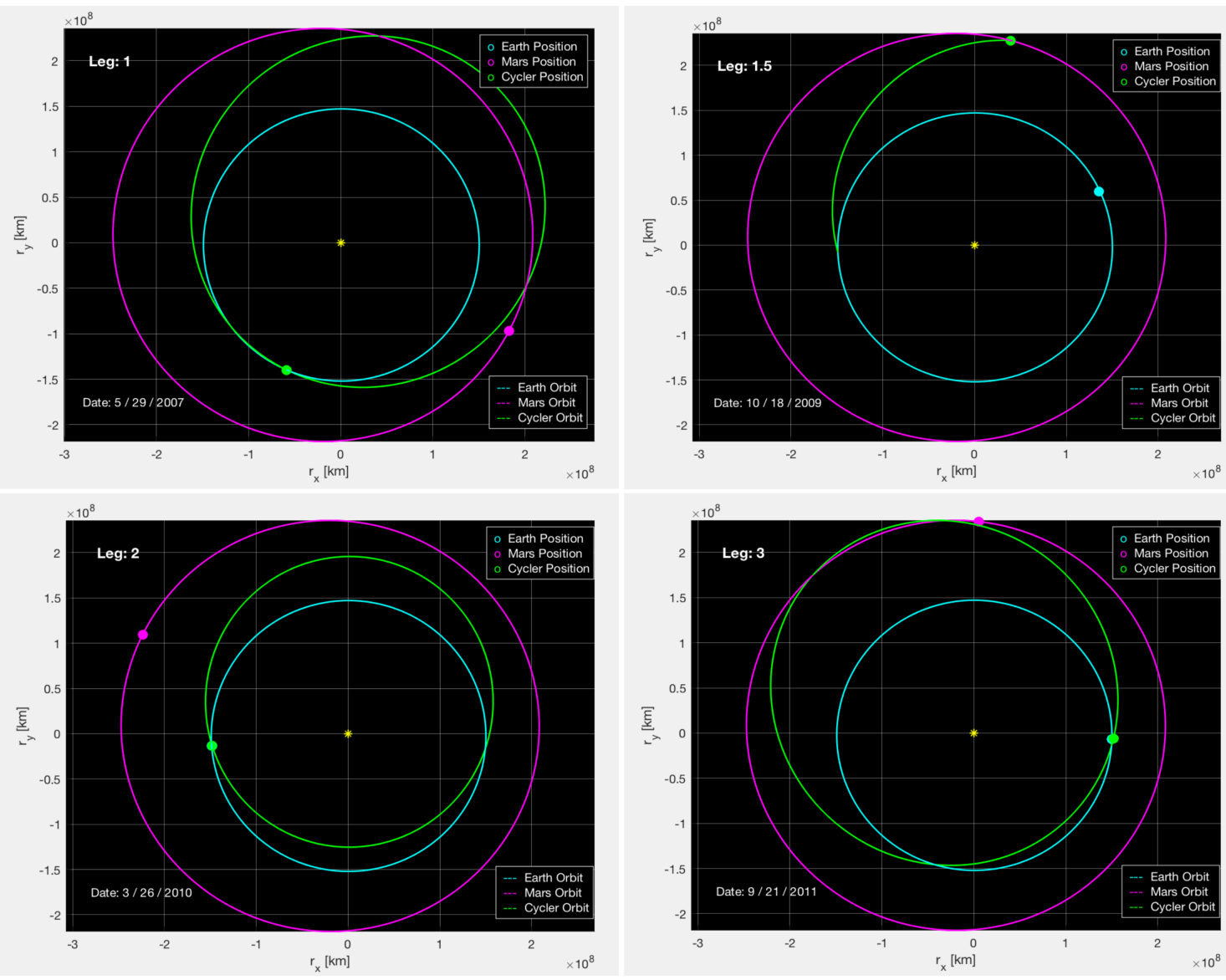

Figure 4.6: First 3 legs of generated inbound cycler 
In order to transition from an Earth-centered orbit to a Sun-centered one, if it is first assumed that the parking orbit of the spacecraft is such that the velocity of the spacecraft relative to the sun is indistinguishable from the velocity of the Earth relative to the sun, the spacecraft would only have to move at $V_{\infty} \mathrm{km} / \mathrm{s}$ faster than the Earth in order to transition onto the cycler; so the only number to consider is the $V_{\infty}$ required at the first leg. As an example, when using the itinerary found in Table 2.1 , the $\Delta V$ value would be $4.01 \mathrm{~km} / \mathrm{s}$. Other values will be presented and compared when discussing test cases in Chapter 6. However, this is not always the case as the shape of the parking orbit can vary widely. In the next chapter, a method to find a parking orbit will be discussed, and the associated $\Delta V$ to burn from the parking orbit onto the cycler trajectory directly will be analyzed. 


\section{Chapter 5}

\section{MANIFOLDS}

The basic principles involved with this section involve the characteristics of the trajectories on the manifolds that will allow the spacecraft to move from Earth onto the cycler trajectory, while attempting to reduce the overall $\Delta V$ involved in the process. This method will then be compared against a normal Lambert's solution transfer.

\subsection{Halo Orbit Insertion}

In order to simplify the following problem, the launch environment and its accompanying aspects will not be analyzed or considered in this work. This leads to the assumption that the spacecraft will start in some parking orbit around Earth and perform a burn from there. The main reasons for this exclusion are that the size, weight, \& other attributes of the spacecraft are largely unknown and most interplanetary spacecraft execute their final burns from parking orbits nevertheless. This allows the $\Delta V$ to be optimized in a much less binding environment; however, the various shapes of parking orbits will be considered both in their performance as well as their practicality.

Before optimizing the shape of the halo orbit, it is first necessary to make sure we can find the trajectory on a stable manifold that would reduce the overall distance from the planet. For pragmatic reasons, the trajectory closest to Earth is the most 'do-able' in terms of the spacecraft arriving at the associated parking orbit. A code developed by Megan Rund[17] was adapted for this work in order to find this trajectory. By giving the function the characteristics of the halo orbit and the propagation time in [TU], the following steps are taken to find the closest trajectory to Earth. 
1. The points in the halo orbit are broadened to allow for quick computation of the various trajectories in the manifold

2. The invariant-stable manifold trajectories are calculated from each point

3. The closest trajectory in the manifold is then found through testing each trajectory

4. The points on the halo are refined to their original quantity for a fine search algorithm

5. The trajectories on the manifold are then calculated for points close to and on either side of the recently found closest trajectory

6. The closest trajectory of that set is found and is used as the output closest trajectory to Earth

7. The necessary parking orbit to reduce the overall $\Delta V$ to burn onto the manifold is then calculated

The outputs of the function give the closest trajectory on the manifold as well as the parking orbit and $\Delta V$ necessary to burn onto the manifold. To visualize the outputs of this process, as southern class halo around L2 with $A_{z}=476083 \mathrm{~km}$ was calculated and utilized for the trajectory optimizing code. Fig. 5.1 gives a representation of the entire trajectory from Earth to halo, while Fig. 5.2 gives a visualization of the parking orbit and its departure. A larger and more detailed figure will be given for the optimized trajectory later on.

For the curious, the outputs and associated parameters of this test are as follows: Parking Orbit Altitude $\left(h_{p}\right)=330.5385 \mathrm{~km}$, Total Time of Flight $(T O F)=197.0438$ days, $\Delta V=3.1629 \mathrm{~km} / \mathrm{s}$. One may notice that the time of flight is very large. This is because relative to the fixed frame, a trajectory on the manifold moves very slowly. A 


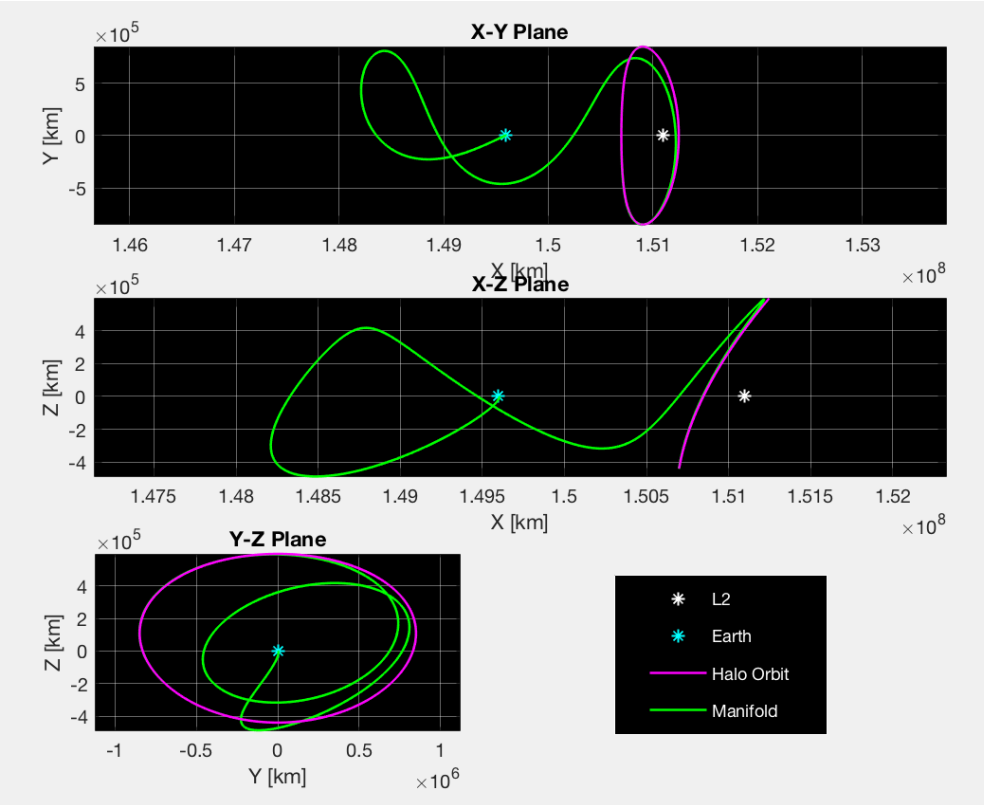

Figure 5.1: Example optimized trajectory for given halo orbit

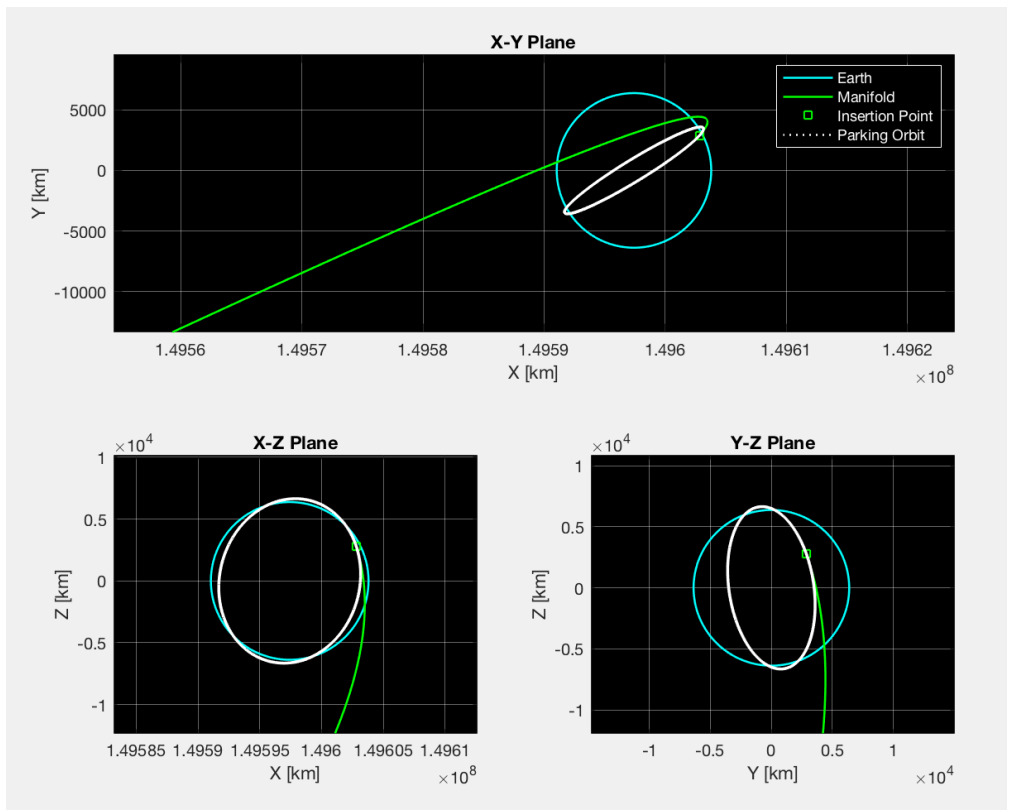

Figure 5.2: Parking orbit to trajectory in manifold 


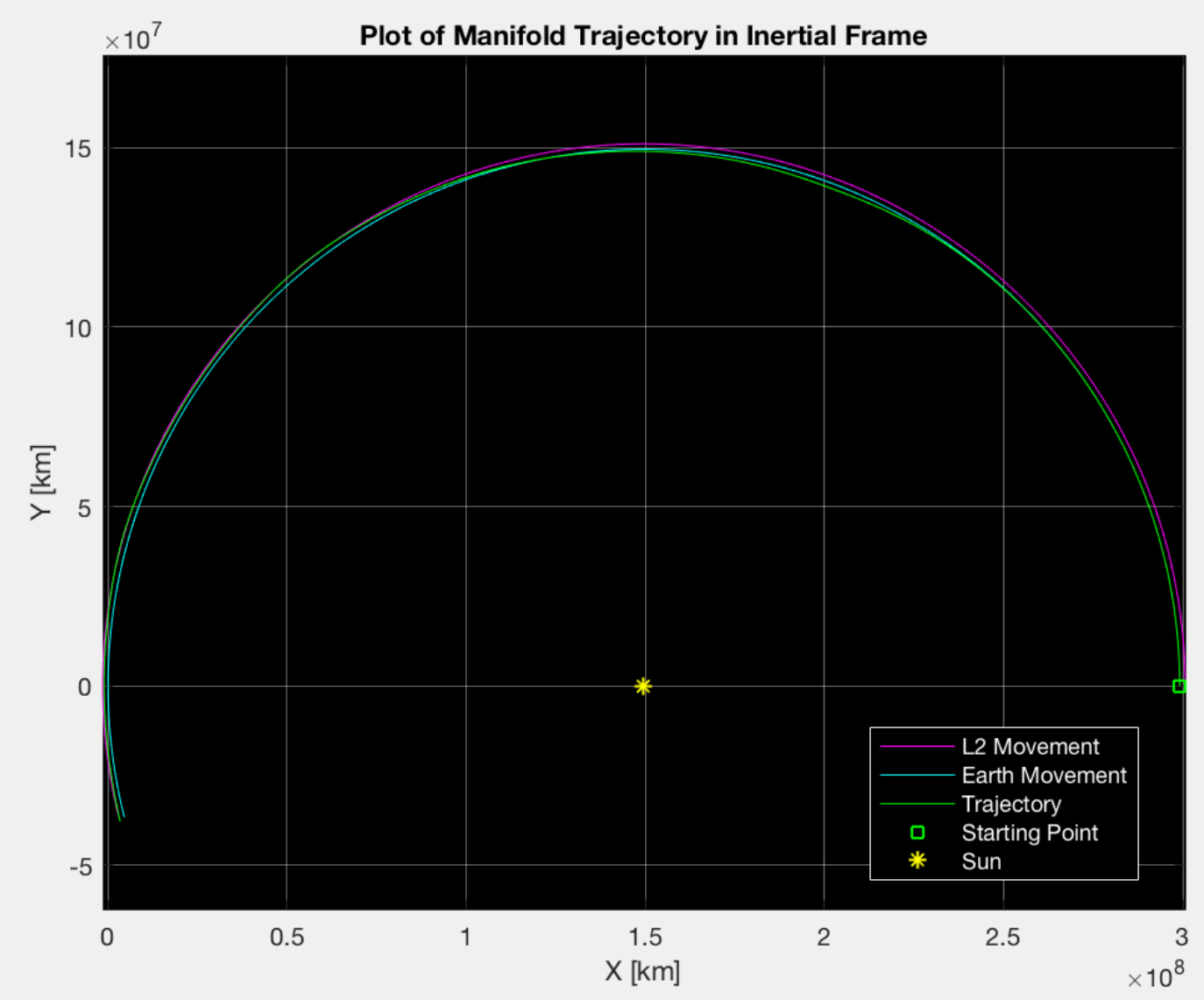

Figure 5.3: Trajectory in manifold in sun-centered inertial frame

relatively unexciting result can be obtained when switching from this fixed frame to the inertial frame. A representation of how the earth, the L2 point, and the spacecraft on the trajectory move over time in the inertial frame can be seen in Fig. 5.3.

\subsubsection{Halo Orbit Optimization}

Now that we have the ability to optimize (albeit via brute-force) a trajectory given a certain halo orbit, the halo orbit shape itself can be optimized in order to further reduce the required $\Delta V$. In order to do this, two separate optimization methods that perform a broad search followed by a fine search were set up. This was done using MatLab's fminsearch optimization function. Though a bit of a departure from the 
previous method of optimization, the author settled on the fact that the nature of halo orbits and their stable manifolds are extremely unpredictable by human standards; thus, attempting to find the proper size of halo orbit would have required an unprecedented amount of trial and error. Allowing an optimization method to 'take care of the dirty work' per se is a much more viable option for this case.

The fminsearch function is a derivative-free method optimizer that operates by giving the user the choice of an initial guess of the solution which is then iterated upon. This allows an optimized solution to reach a local minima. To mitigate this and allow for a global minimum to be obtained, the optimization is run at various initial values which are then compared later. Although this is a multi-dimensional optimizer, the only input will be the size of the halo or $A_{z}$. The classes of the halo orbit were not considered, as it does not matter in this case. The default parameter chosen was the southern class.

In order to attain the range of optimized solutions, five separate optimizations with values of $A_{z}=\{3 E 5 \rightarrow 7 E 5 \mid 1 E 5\} \mathrm{km}$ were produced. These values were derived from known possible values for the size of halo orbits around L2 without pushing the limits of what is possible. The optimization was also only allowed to run for 20 iterations to view the trend of the optimization and avoid optimizing on values with very low change. An example of the optimization iterations for $A_{z 0}=7 E 5$ is given in Fig. 5.4. In conjunction an organization of the results is given in Table 5.1.

The obvious trend that most will notice is that increasing size of the halo orbit generally decreases the magnitude of the $\Delta V$. While this trend may seem favorable, it is also important to look at both the amount of time that the spacecraft will spend on this trajectory, as well as the parking orbit that is associated with each trajectory. Though the focus of this work lies in reducing the $\Delta V$ for cycler trajectory insertion, it is vital not to ignore practicality along the way. For the purposes of this work, any 

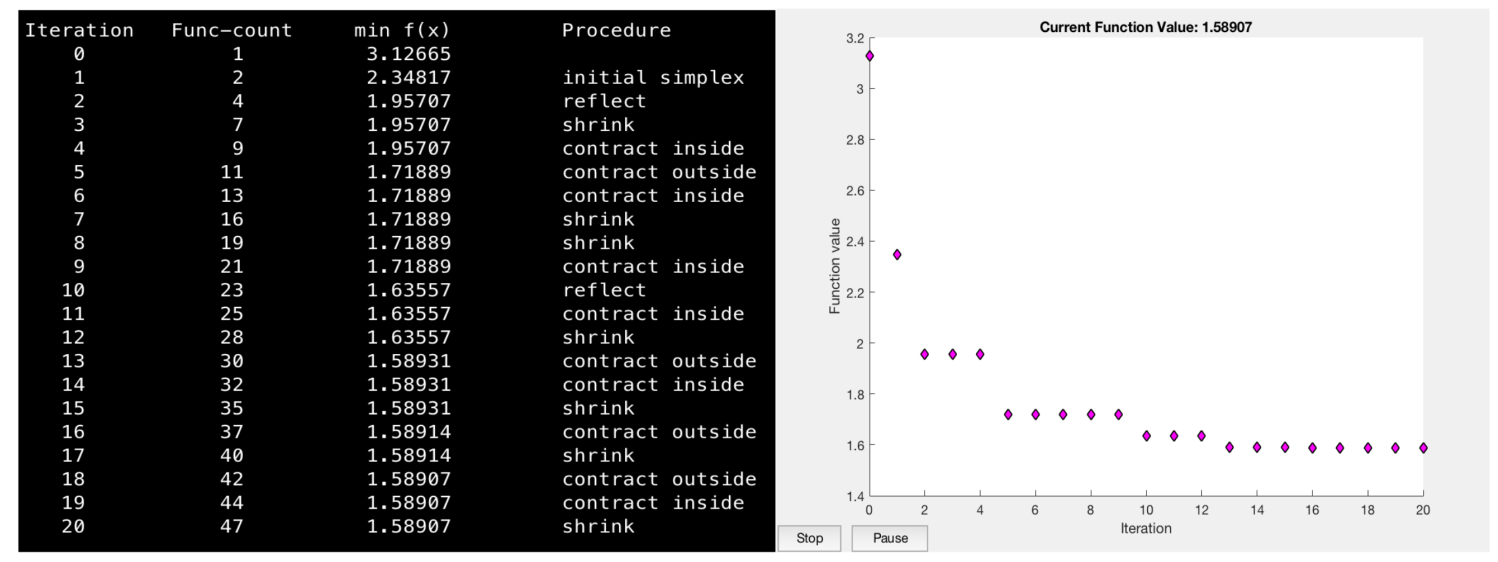

Figure 5.4: Optimization example for $A_{z}=7 * 10^{5} \mathrm{~km}$

Table 5.1: Optmization results for various halo orbit regimes

\begin{tabular}{c|cccc}
\hline$A_{z 0}$ & $A_{z}$ & $\Delta V$ & $h_{p}$ & TOF \\
{$\left[10^{5} \mathrm{~km}\right]$} & {$\left[10^{5} \mathrm{~km}\right]$} & {$[\mathrm{km} / \mathrm{s}]$} & {$[\mathrm{km}]$} & {$[$ days $]$} \\
\hline 3 & 2.999707 & 3.18390 & 235 & 197 \\
4 & 4.197894 & 2.75254 & 2413 & 31 \\
5 & 5.183945 & 3.08510 & 353 & 169 \\
6 & 6.300286 & 2.31501 & 5799 & 738 \\
7 & 7.746880 & 1.58907 & 11042 & 596 \\
\hline
\end{tabular}


transfer that takes more than a year, or any parking orbit higher than lower MEO will deem the manifold transfer unfit for consideration. The time constraint is chosen based on the fact that the cycler trajectory will already need to be operable for a significant amount of time ( $~ 33$ years), and any longer than that is beyond what most spacecraft are conceivably capable of. The parking orbit constraint is derived from the basic fact that LEO is the most accessible orbital regime around Earth much higher than lower MEO can be costly; even if the cycler spacecraft were to be built in orbit, LEO to lower MEO is still the most viable option.

Though a strict weighting system was not considered, one of the most obvious choices for the halo orbit lies in the $400,000 \mathrm{~km}$ regime with a reasonable parking orbit, relatively low $\Delta V$, and a very favorable transfer time. In an attempt to find the best solution in this locality, the value $A_{z}=4.197894 E 5 \mathrm{~km}$ was plugged back into the optimization and run until the default tolerances of the optimization were met (1E-4). Though not much of a change, the $\Delta V$ cost was lowered to $2.7513 \mathrm{~km} / \mathrm{s}$. Furthermore the new parameters are $A_{z}=4.197279 E 5 \mathrm{~km}, T O F=172$ days, and $h_{p}=2430 \mathrm{~km}$. Despite the efforts of the optimizer, the change in TOF compared to the $\Delta V$ saved was displeasing, and therefore it was decided that the original halo orbit was sufficient.

A diagram of the halo orbit and trajectory are given in Fig. 5.5. This halo orbit will now act as the departure point of our unstable manifolds required for cycler trajectory insertion. This trajectory will also be shown in the inertial frame at a later time when discussing the spacecraft's entire trajectory. Furthermore, a view of the new parking orbit is also available in Fig. 5.6.

As a quick note, it is possible to launch directly from Earth into the stable manifold, removing the need entirely for a parking orbit around Earth. This allows the possibility of saving some of the required $\Delta V$ to arrive at the halo orbit. This will 

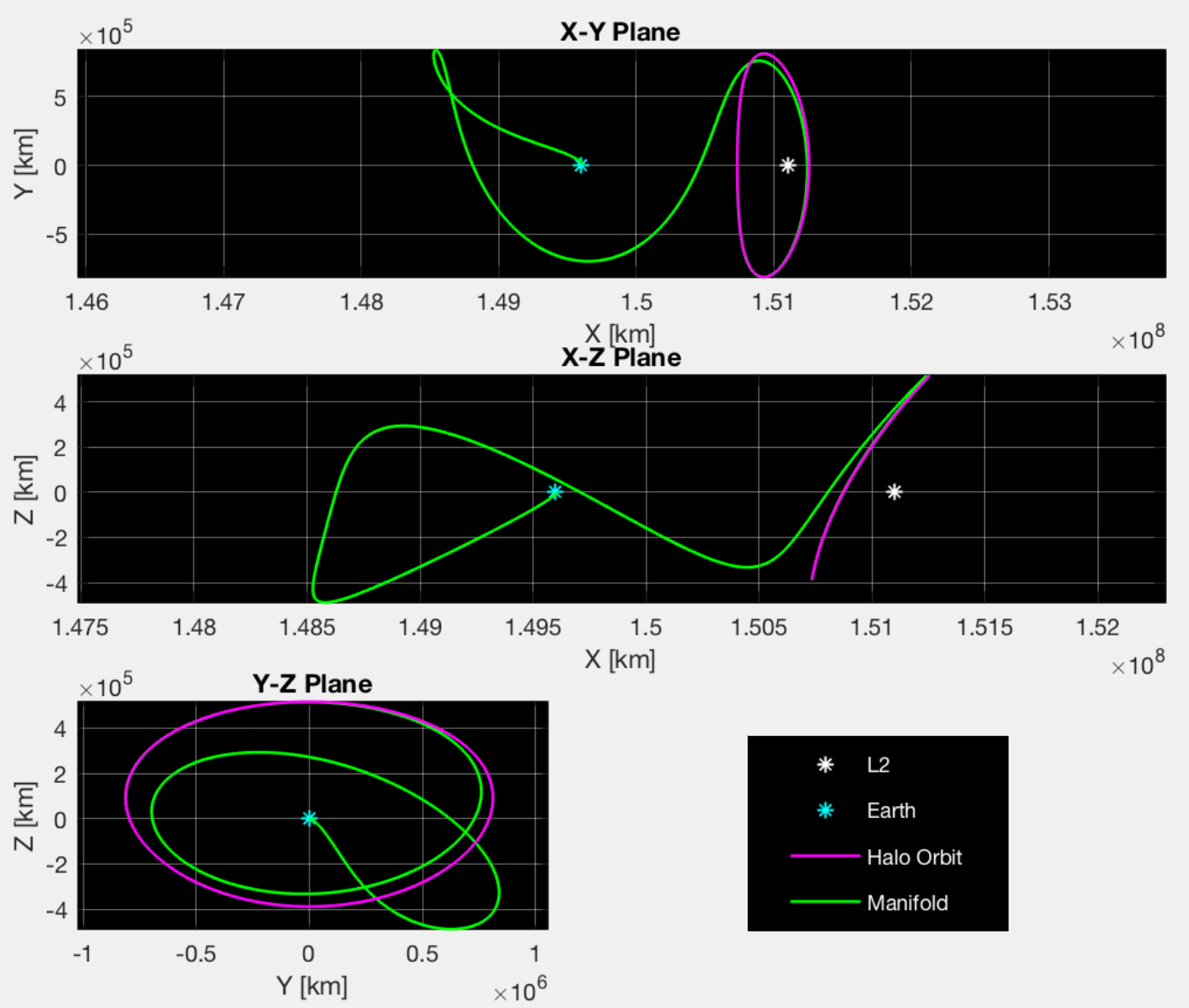

Figure 5.5: Optimized halo orbit and stable manifold trajectory

however not be considered for the purposes of further calculation, but may be important to keep in mind when considering the final $\Delta V$ values for each method.

Similarly, it is possible to burn directly from the parking orbit onto the cycler trajectory via direct inject; considerably changing the assumed $\Delta V$ to burn onto the cycler (when using the nominal $V_{\infty}$ value at Earth). Using the parking orbit presented for this particular manifold, the largest and smallest required $\Delta V$ to burn onto the cycler are $4.52 \mathrm{~km} / \mathrm{s}$ and $4.01 \mathrm{~km} / \mathrm{s}$ respectively, with an average of $4.26 \mathrm{~km} / \mathrm{s}$. These values will be set aside for now and considered further when discussing the total $\Delta V$ via the manifold method. 

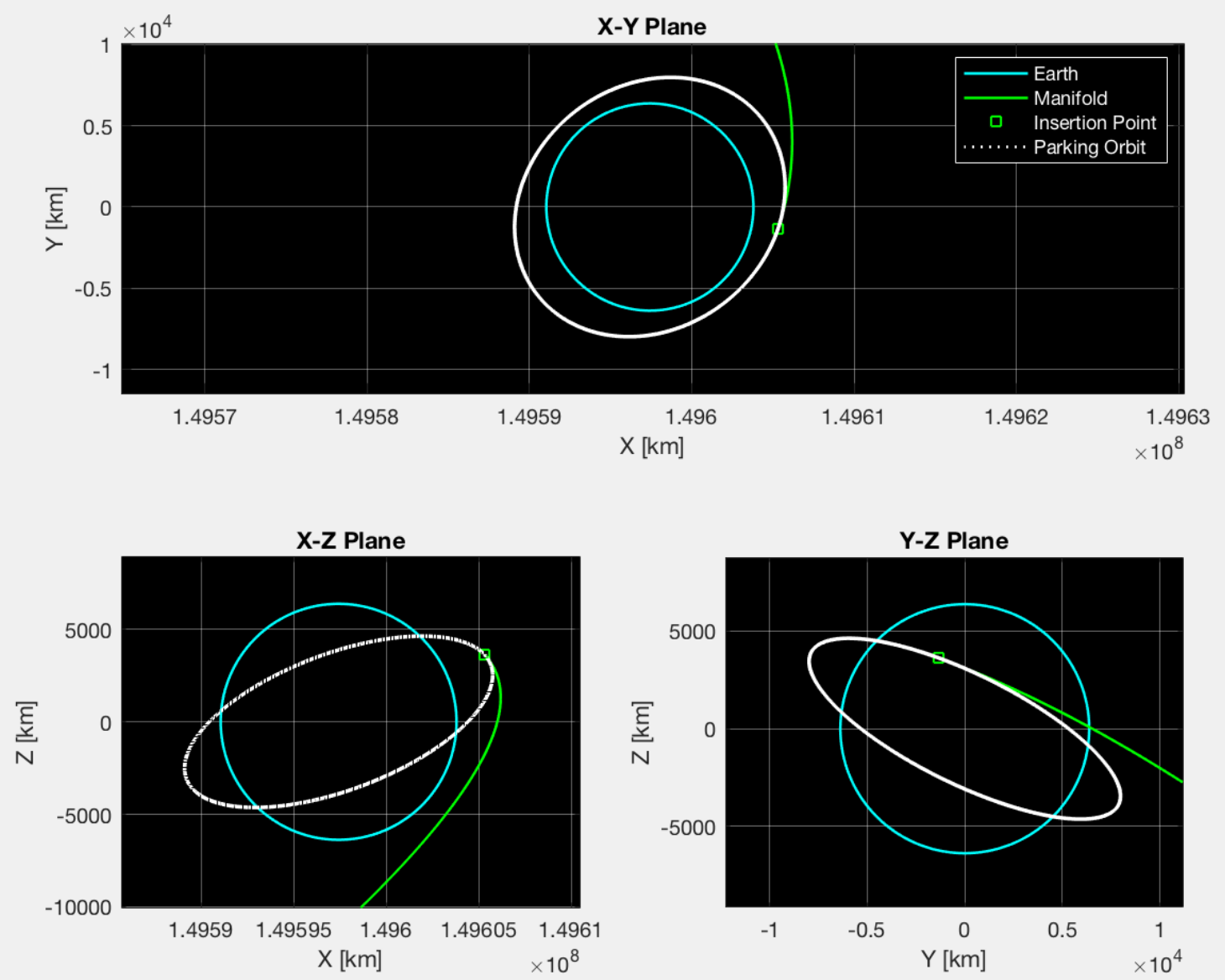

Figure 5.6: Optimized solution parking orbit and trajectory 


\subsection{Halo Orbit Departure}

Now that the spacecraft is on the halo orbit, it is possible to slightly perturb the periodic trajectory and enter onto a trajectory in the unstable manifold. This will ultimately allow the spacecraft to perform cycler trajectory insertion.

In order to perform this maneuver, a process similar to the halo orbit insertion method wad developed. The central difference between the two however, is that it calculates which trajectory passes closest to the first leg of the cycler, rather than Earth itself. Also, the halo orbit will no longer need to be optimized or even calculated, since that was taken care of previously. However, the unstable manifold will be optimized via similar methods to the halo orbit; this optimization process will be much more refined however. This is primarily due to the fact the trends of the unstable manifolds are far more predictable and adaptable.

It is also extremely important to mention that the circular assumption of Earth's orbit for the purposes of the CR3BP will continue to be in effect until the transfer to the first leg of the cycler occurs. While this may seem dubious at first, since the orbit of Earth is far more circular than Mars, this does not hinder or breach the dynamics involved in the transition between operating environments. To illustrate this fact, the orbits of Earth and Mars in the circular and ephemeris models can be seen in Fig. 5.7. It is clear that Earth's orbit is 'circular enough' to allow for this assumption to hold. Moreover, this assumption allows all operations to be contained in the $x-y$ plane for the duration of this maneuver.

\subsubsection{Time of Departure}

Unfortunately, with this system the arrival date at Mars is very specific, and thus the first leg of the cycler has a specific shape and location. In order to obtain the 


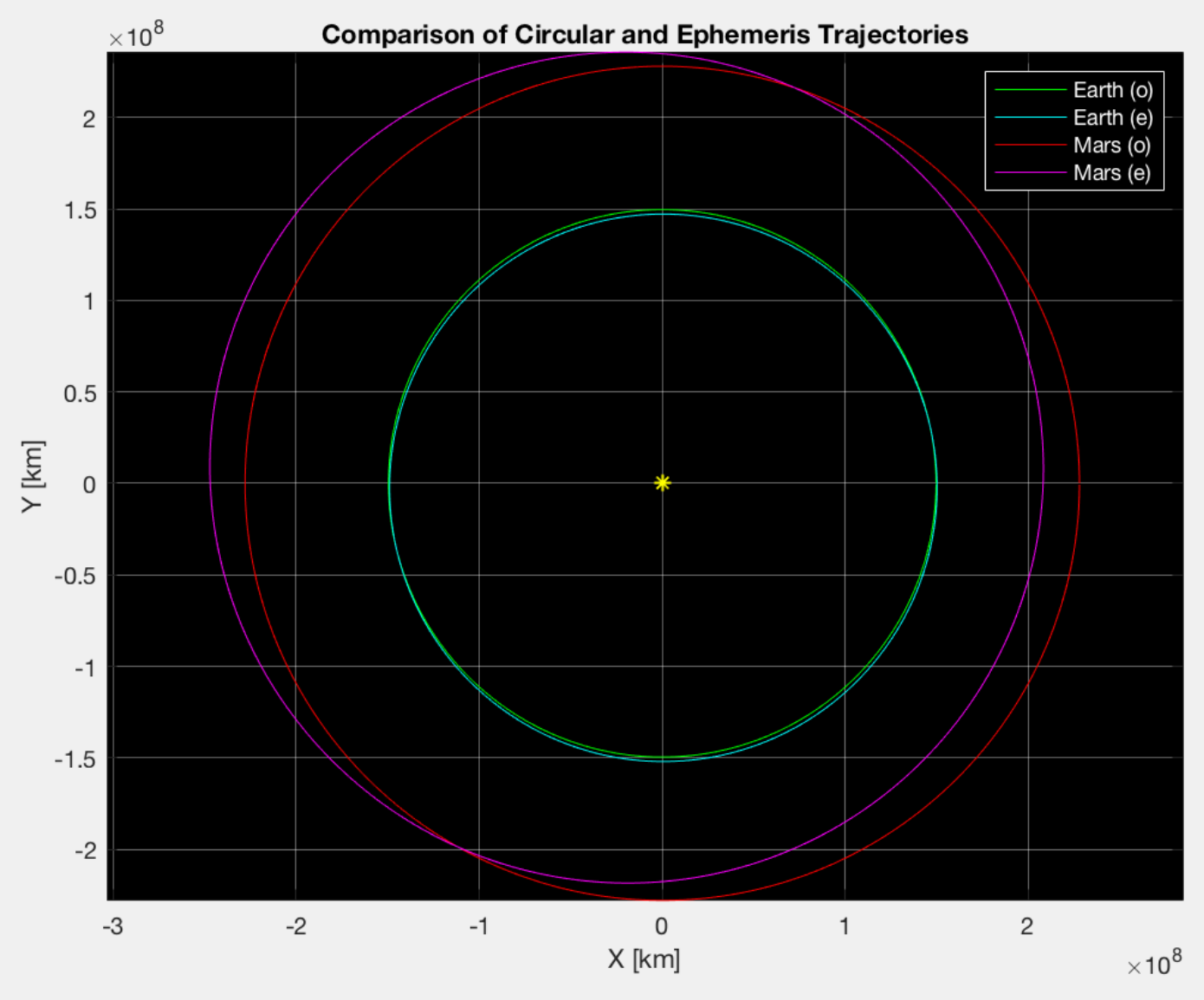

Figure 5.7: Earth and Mars circular vs. ephemeris orbit shapes 
best result possible, it is necessary to make sure that the departure date of the halo orbit is sufficient in getting a trajectory on the unstable manifold adequately near that first leg. This first necessitates a test for the relationship between transfer times and distances travelled on the unstable manifold. Since we are using the invariant manifold and circular solar model, we can do this process at any departure time and simply shift the departure date via a frame rotation as necessary, a process that will be demonstrated later. For the purposes of the following demonstrations and explanations, the outbound cycler found in Table 2.1 will be used as the example first leg of a cycler trajectory.

Following the self-imposed constraint of keeping these transfers to under a year, the remaining 334 days or about 6 TU (subtracting 31 days for the first transfer) of the year were allowed to propagate the unstable manifold. For the purposes of visualization, both the inertial frame and fixed frame are plotted in Fig. 5.8 and Fig. 5.9 respectively.

From the diagrams one can see that while some trajectories on the manifold do not venture too far from the halo orbit, others are able to veer outwards and decently far away from Earth. One may also see that the manifold does not 'lag' behind the orbit of Earth, essentially meaning that the remaining 334 days is plenty of time to cross the path of the cycler from any halo departure date. Finally, it is also noticeable that these trajectories seem to exhibit much more predictable behavior; something that will prove useful when performing frame rotations.

A key characteristic of these trajectories is that no matter what date is chosen to depart the halo, the shape of the manifold remains consistent, and the frame is simply shifted in angle around the $z$-axis. This allows for a much smoother process when choosing the desired trajectory on the unstable manifold. By simply overlaying the first leg of the cycler trajectory onto the manifold space, it is possible to test 


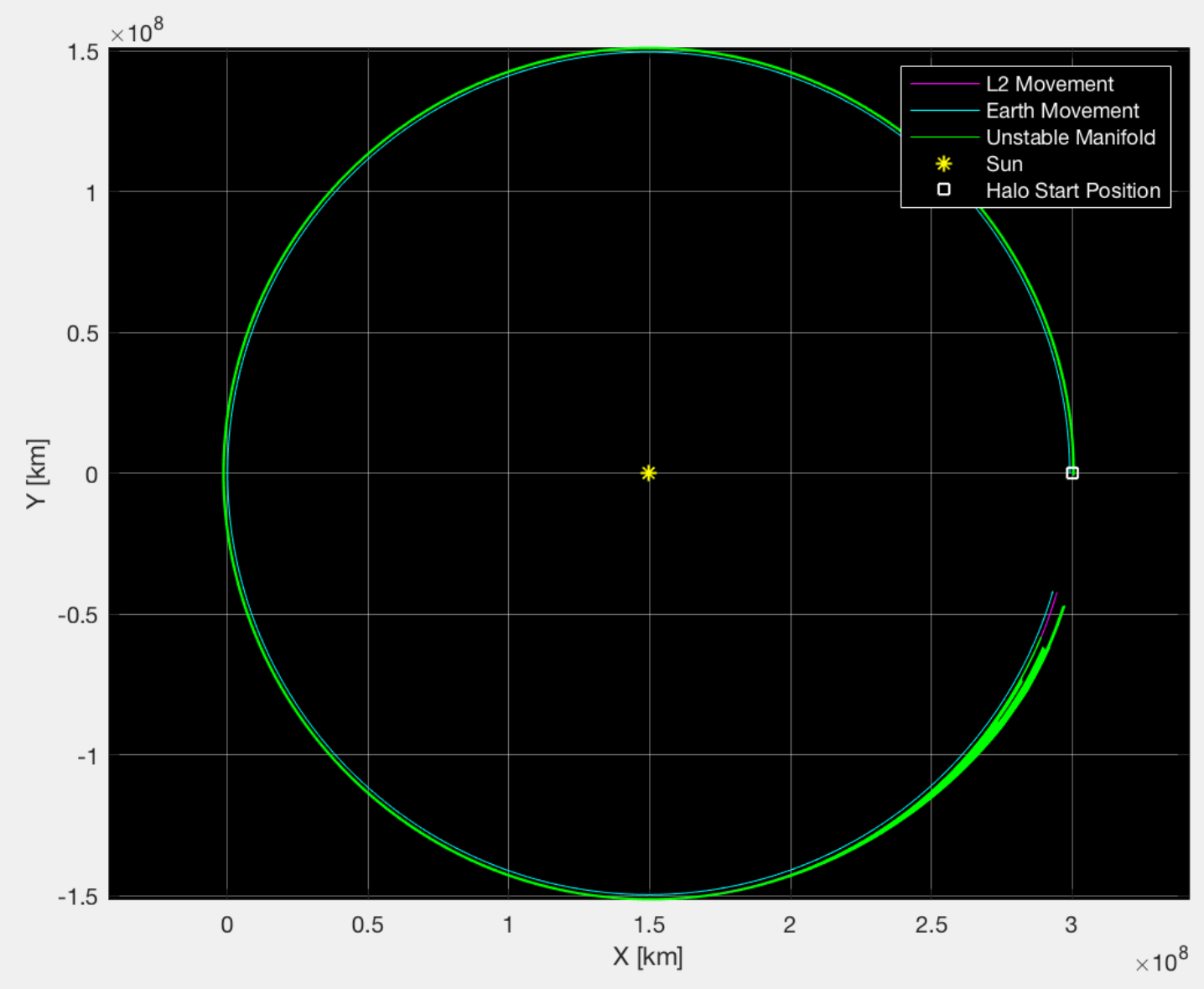

Figure 5.8: Unstable manifold over 6 TU in inertial frame 

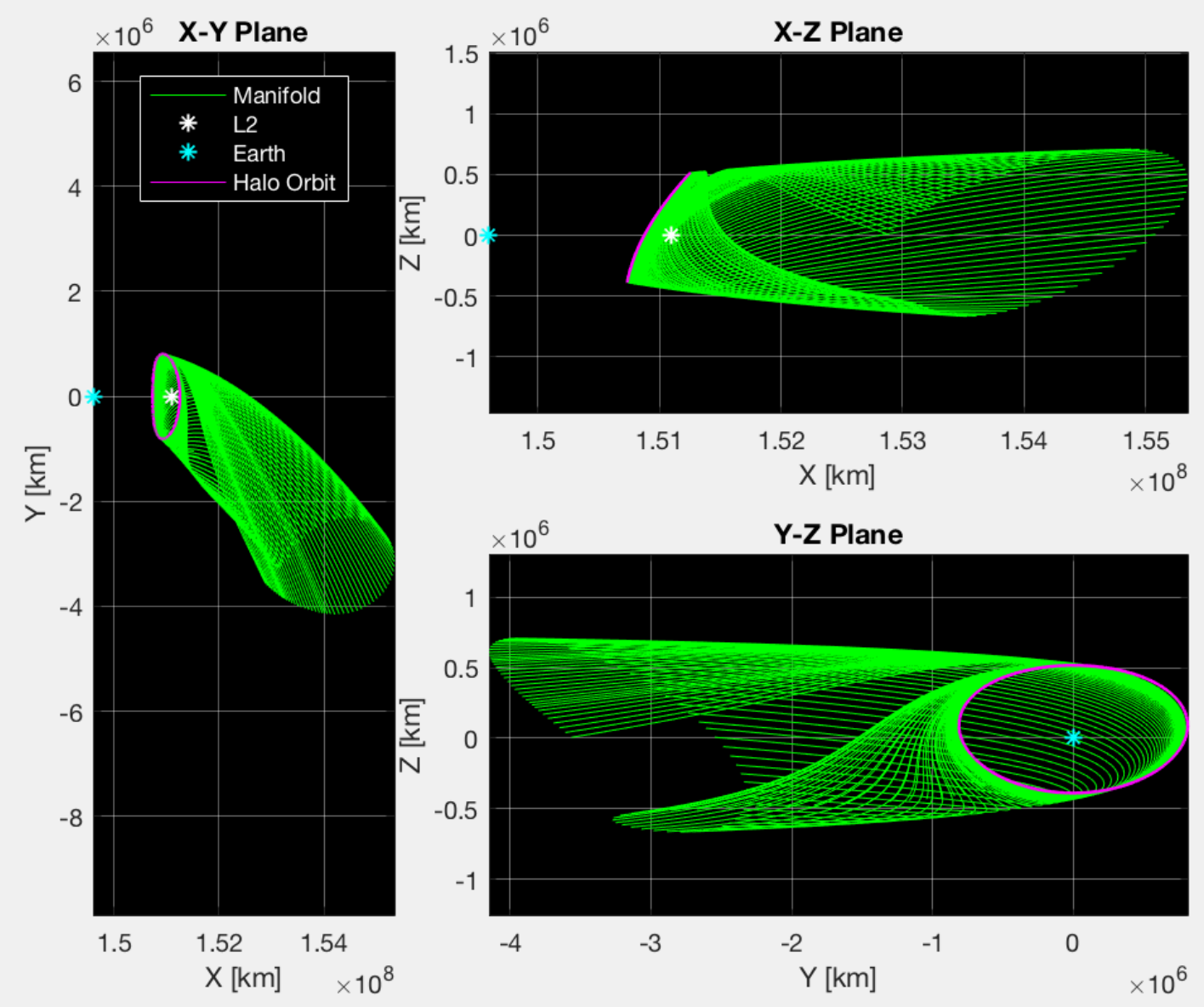

Figure 5.9: Unstable Manifold over 6 TU in fixed frame 
for trajectories on the manifold that intersect (or get very close to) the cycler. The departure date of the halo can then be shifted (via an angular rotation of the plot itself) and tested further until an optimal solution is found. An algorithm that uses exactly this approach was developed and takes the following steps:

1. The number of points in a given halo orbit are broadened, similar to the halo orbit optimization

2. The corresponding trajectories on the unstable manifold are calculated and transformed into the inertial frame with initial angular rotation, $\theta_{z}$ of $0 \mathrm{rad}$

3. The first leg of a cycler trajectory is then compared to each trajectory on the manifold to find the distances, $d_{c}$, between them

4. Similarly, the required $\Delta V$ to burn onto the cycler trajectory is also calculated through simple vector comparison

(a) Note that this $\Delta V$ can be deceptive. Since only the velocity vectors are compared, this value is only true if the cycler trajectory and manifold trajectory intersect. We will keep this in mind for later.

5. The $d_{c}$ and $\Delta V$ values are used to develop a weighted score for the overall performance of the maneuver

6. The manifold is then rotated in frame by some $\Delta \theta_{z}$ (via the optimizer's choice) and the process is repeated until tolerance is met on the optimizer.

7. The output of the optimizer is the $\theta_{z}$ that produces the lowest score. This angle is then chosen as the basis for a fine search optimization

8. The optimization is re-run (if necessary) until the best value for $\theta_{z}$ is found 


\section{Table 5.2: Table of example output values for frame rotation}

\begin{tabular}{c|cccccc}
\hline$\theta_{z}[\mathrm{rad}]$ & 0 & $\frac{\pi}{3}$ & $\frac{2 \pi}{3}$ & $\pi$ & $\frac{4 \pi}{3}$ & $\frac{5 \pi}{3}$ \\
\hline$\Delta V[\mathrm{~km} / \mathrm{s}]$ & 3.38 & 3.63 & 3.64 & 3.57 & 3.62 & 3.62 \\
$d_{c}\left[10^{4} \mathrm{~km}\right]$ & 0.57 & 3.29 & 4.01 & 4.05 & 3.55 & 3.75 \\
score $[-]$ & 1.41 & 3.39 & 3.90 & 3.97 & 3.57 & 3.71 \\
\hline
\end{tabular}

Before discussing the optimizer, the generally expected outputs that the system expects for various $\theta_{z}$ values will first be illustrated. This is for the purposes of understanding the score associated with each value. To demonstrate this process, values of $\theta_{z}=\left\{0 \rightarrow \frac{5 \pi}{3}\right\}$ with $\Delta \theta_{z}=\frac{\pi}{6}$ were used. In order to determine the most optimum trajectory on the manifold, the $\Delta V$ and $d_{c}$ values are compared and weighted to give a score to be minimized. This score is given by the following equation:

$$
\text { score }=\frac{w_{1} d_{c}}{10^{3}}+w_{2} \Delta V
$$

where $w_{1}=0.7$ and $w_{2}=0.3$ are the weights of importance given to each parameter. The rational for weighting the distance higher than the magnitude of the burn is due to the fact that getting a trajectory on the manifold as close as possible to the cycler trajectory allows for a direct burn and does not require a Lambert's solution to patch the conics. Furthermore, this greatly simplifies the metrics necessary to determine the best trajectory.

A table of associated values with each angle are provided in Table 5.2. To spare the reader of an influx of figures, Fig. 5.10 displays an example result $\left(\theta_{z}=\frac{2 \pi}{3}\right)$ from this list. This is meant solely for visual purposes and is aimed at showing the first leg of the cycler trajectory as well as the departure region in the inertial frame; a much more detailed figure of the final, optimized trajectory will be presented later.

To perform the optimization, a slightly different algorithm than that used in optimizing the halo orbit was used. Matlab's fminbnd function was used because of 


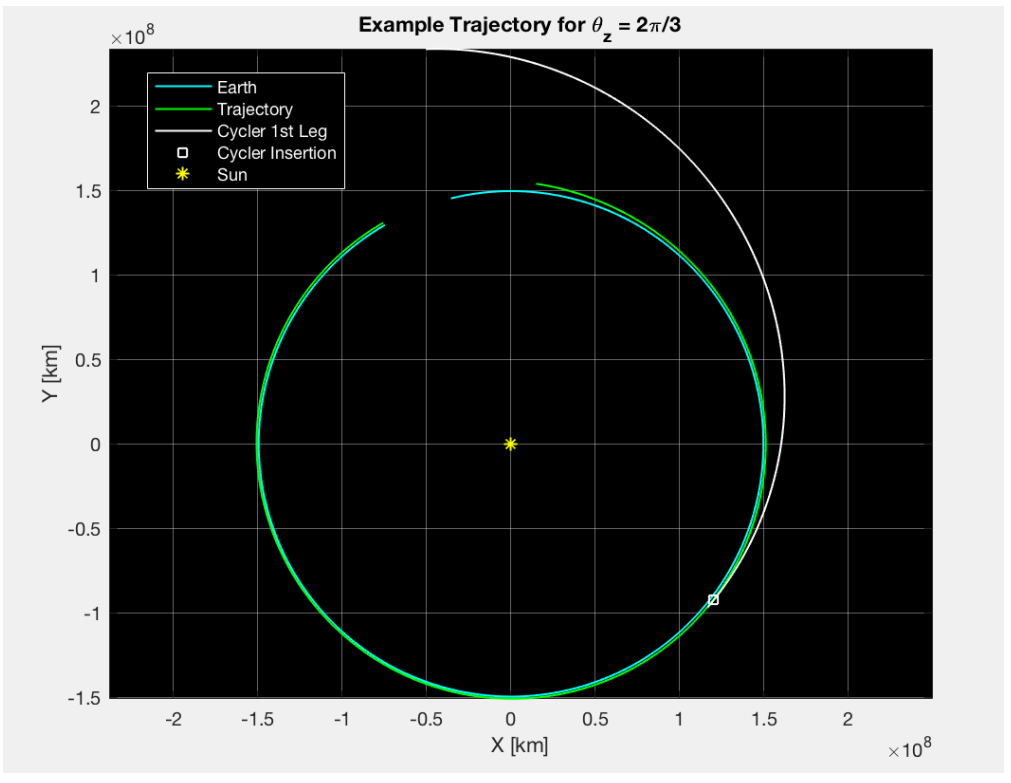

\section{Figure 5.10: Example of unstable manifold trajectory}

its capacity to allow the user to input bounds for the the optimizer to search between, rather than a single value to be iterated upon. This means that the initial guess for the optimized value does not have to be a 'good guess'.

The optimizer implemented the aforementioned algorithm by testing various values for $\theta_{z}$ and optimizing the associated score. The bounds used as the input were $\theta_{z}=(0,2 \pi)$. As a slight fault of the optimizing function, there is a tenancy to not test the limits of these bounds, therefore, a second, similar optimization was run simultaneously with $\theta_{z}=(-\pi, \pi)$. The results of each optimization can be visualized in Fig. 5.11 and Fig. 5.12 respectively.

Both optimizations performed competently, arriving at similar solutions for $\theta_{z}$; with the best value of $0.741596 \mathrm{rads}(\sim \pi / 4)$ and a score of 1.0639. This is already far better than most of the scores in our table of examples, so this method seems promising so far. For the inquisitive, the distance, $d_{c}=1500 \mathrm{~km}$ and the $\Delta V=3.51$ $\mathrm{km} / \mathrm{s}$.

To perform the second search, the halo orbit is refined to its original resolution and 


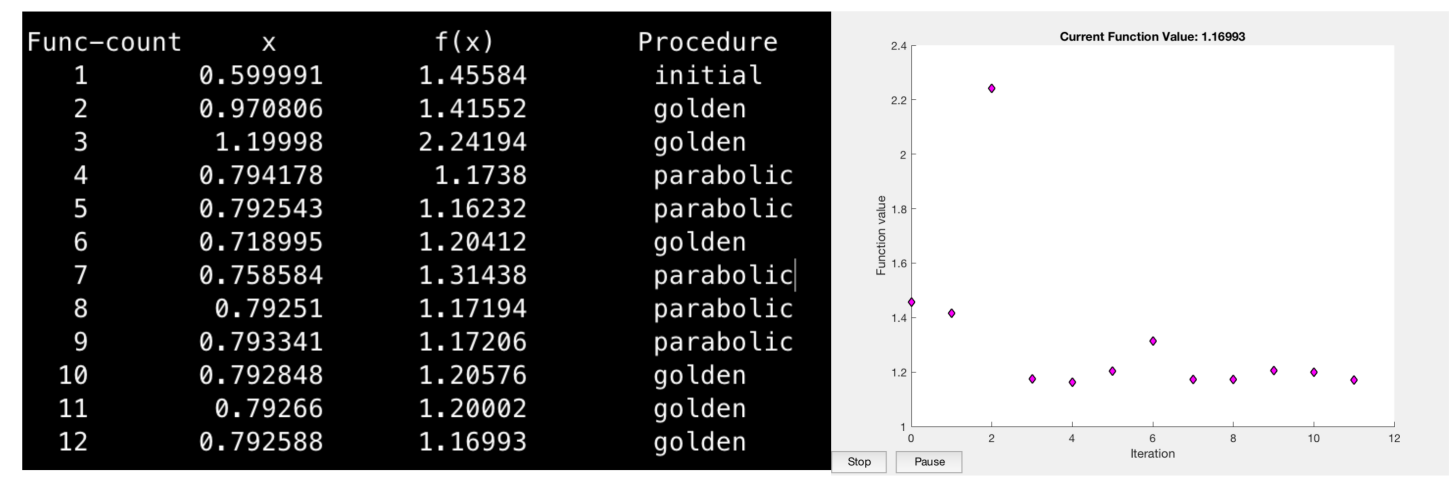

Figure 5.11: First halo optimization $\mid \theta_{z}=(0,2 \pi)$

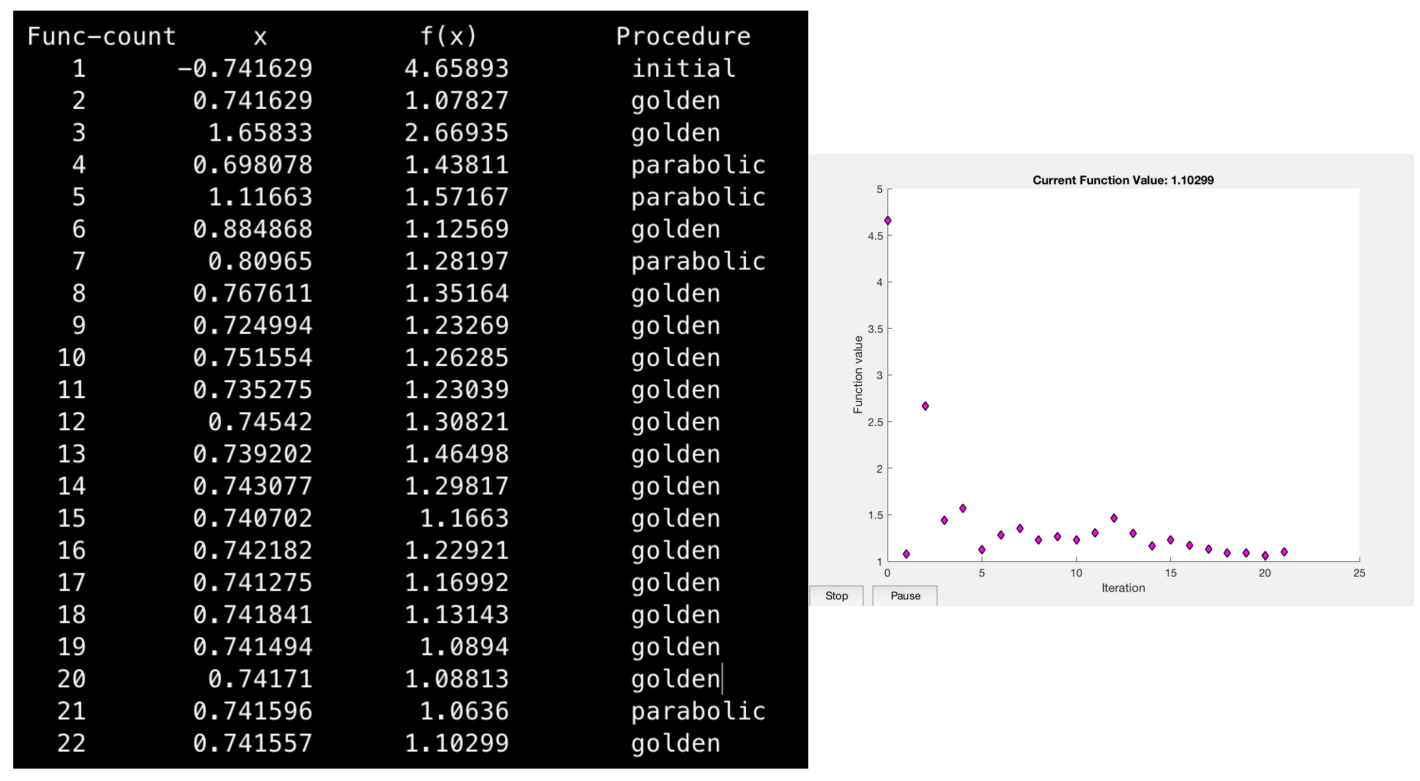

Figure 5.12: First halo optimization $\mid \theta_{z}=(-\pi, \pi)$ 
the known solution is used to find any solutions in that region that may perform more preferentially. As a side note, it was found that a second, fine search optimization, was not necessary to refine the input angle and lower the score since the tolerances of the previous optimization are so strict. With that being said, the value for $\theta_{z}$ was plugged into the algorithm at full manifold fidelity and run a final time. Staggeringly, the score dropped to a value of 0.91 , with $d_{c}=132 \mathrm{~km}$ and $\Delta V=3.02 \mathrm{~km} / \mathrm{s}$.

Our total $\Delta V$ for the entire trajectory from parking orbit to cycler insertion is now $5.77 \mathrm{~km} / \mathrm{s}$. Unfortunately, this is not lower than the value of $4.01 \mathrm{~km} / \mathrm{s}$ that we were trying to overcome, however the feasibility of this maneuver has been demonstrated. Furthermore, in the following chapter, various results of a few more manifold and cycler combinations will be demonstrated, which will hopefully give insight in to whether or not this maneuver can be used to save $\Delta V$. It is also worthy to note that the first leg of a cycler trajectory is not necessarily the only start available for the trajectory. Any leg that starts at Earth can be used as the initial point and many of these $V_{\infty}$ values are much higher than our value of $5.77 \mathrm{~km} / \mathrm{s}$. This means that depending on the launch date, this maneuver may be useful.

For visual purposes Fig. 5.13 displays a plot of the entire trajectory from parking orbit to cycler insertion in the synodic frame. It was chosen not to show just the unstable manifold portion here in the synodic frame as it is mostly unhelpful. To remedy this, a figure of the unstable portion of the trajectory in the inertial frame is given in Fig. 5.14. More plots similar to these will be given in the following chapter discussing various results. 

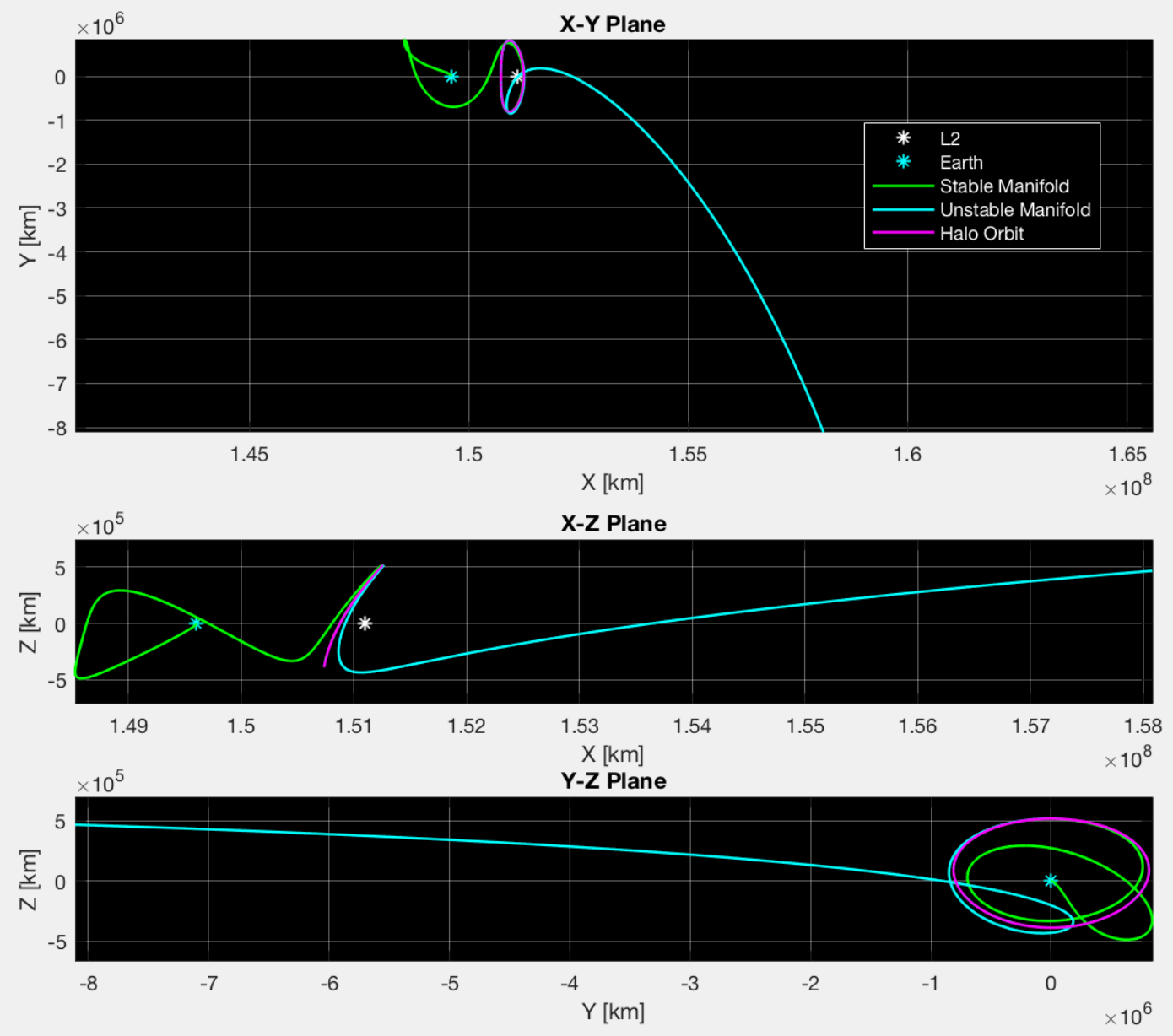

Figure 5.13: Entire trajectory from parking orbit to cycler insertion in synodic frame 


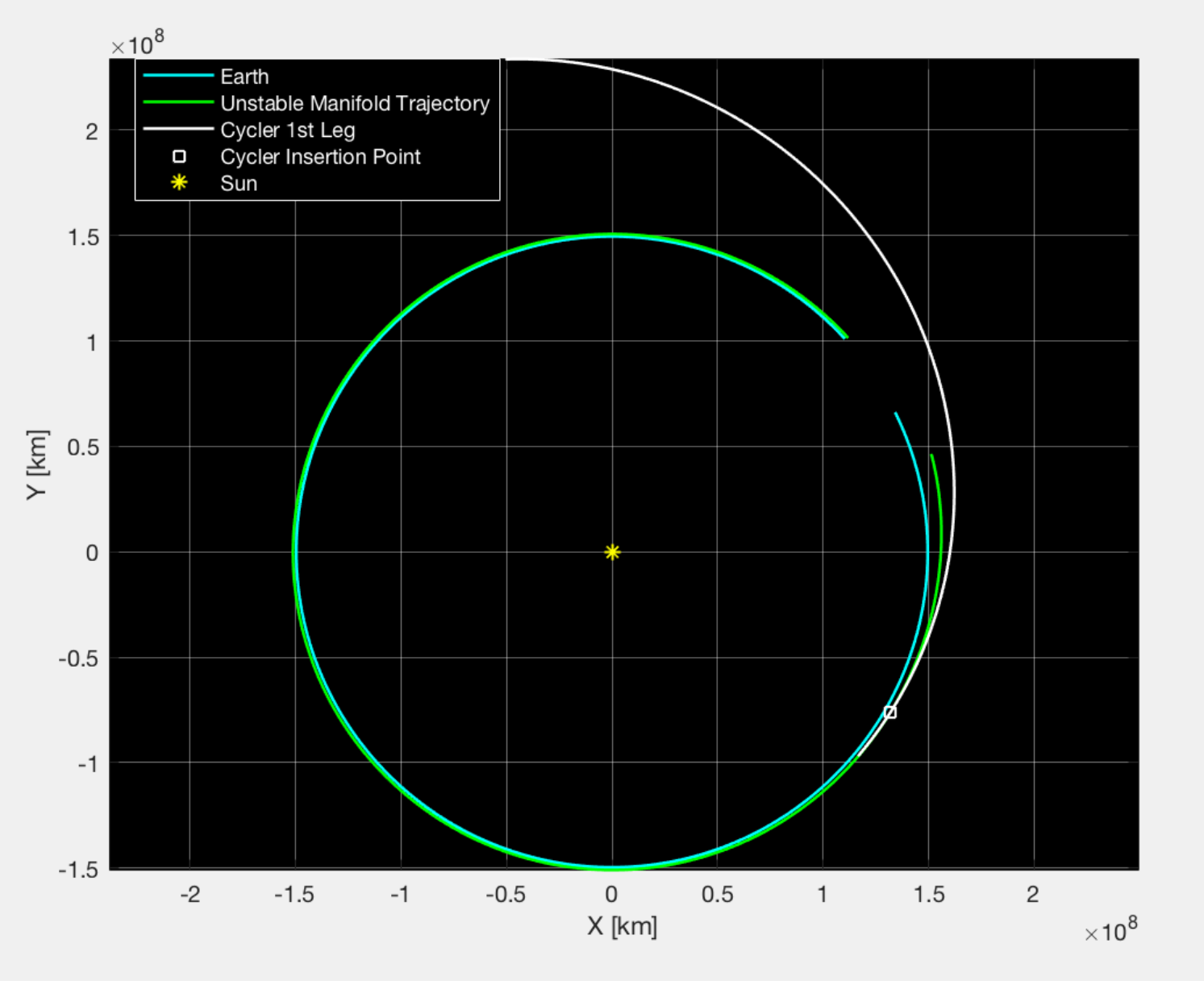

Figure 5.14: Unstable manifold trajectory to cycler insertion in inertial frame 


\section{Chapter 6}

\section{DISCUSSION OF VARIOUS TEST CASES}

This chapter will discuss and analyze some of the results obtained using the methods discussed throughout the entire work for various testing cases. These results will be compared on a few parameters such as feasibility, desirability, and complexity.

All known cycler trajectories, both provided to the author and generated by the author will be used, and all unstable manifolds will be calculated and optimized just as before. However, the parking to halo orbit will not be reanalyzed as this was already determined to be the most desirable case for halo insertion. It should be pointed out that the processes investigated in previous sections are mostly automated

so that will not be reanalyzed here for each case. Instead the final result and its properties/consequences will be discussed.

\subsection{Nominal Cases}

For the first 3 cases, the algorithm developed in the previous chapter for 3 other cycler trajectories was run, those being the inbound cycler provided by T. McConaghy[15], and the outbound \& inbound cyclers that were generated in Chapter 4. While many of these results are similar and relatively unexciting, they do provide insight into the consistency of these transfers.

All three cases involve the S1L1-B cycler trajectory, simply for the various launch dates known. In the last chapter, an outbound cycler (T. McConaghy) was used as a working example and will hereafter be referred to as Outbound-1. This chapter will go over the remaining results of the Inbound-1 vehicle (see Appendix A for itinerary) as well as the generated outbound and inbound trajectories, which will be called 


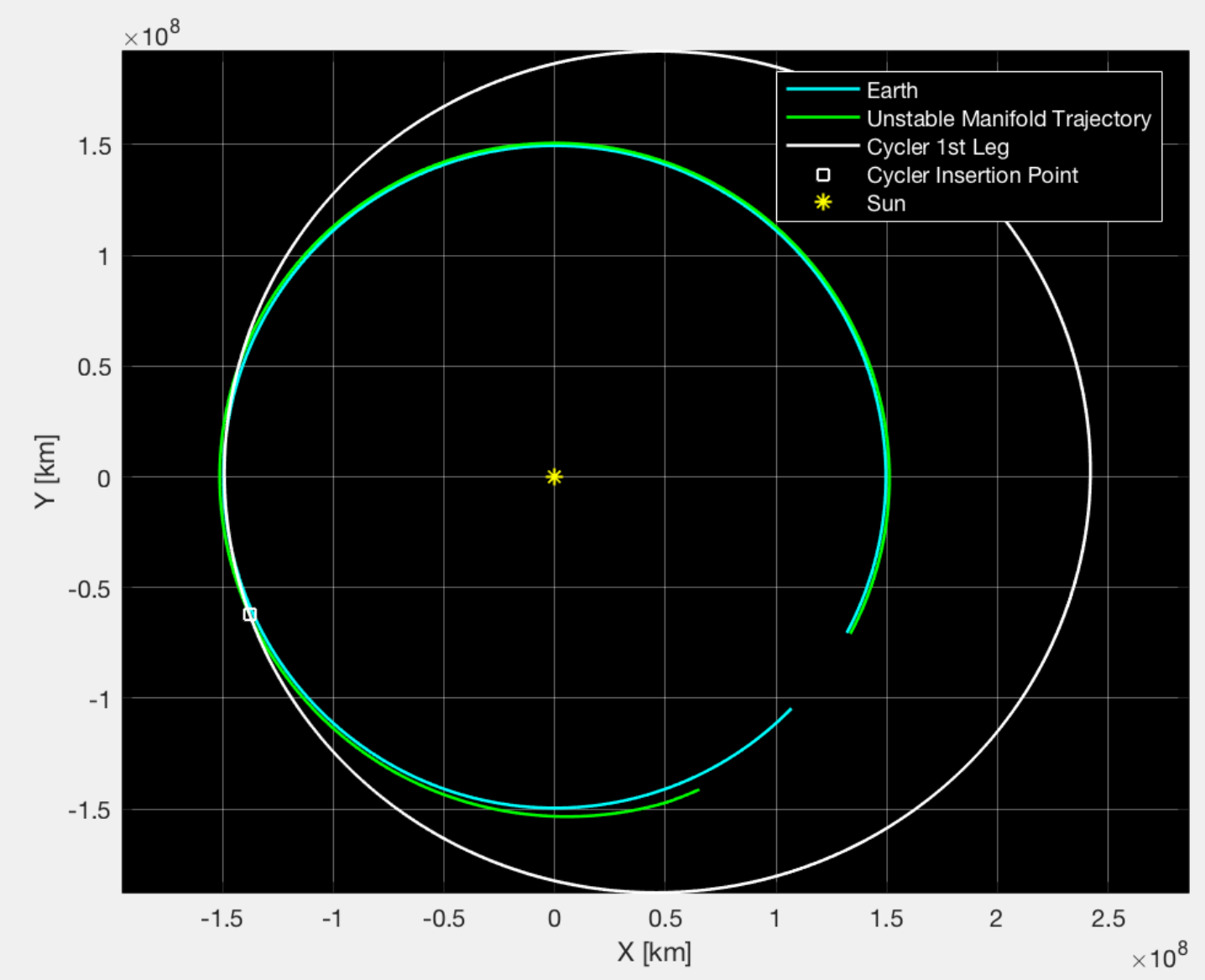

Figure 6.1: Inbound vehicle 1 results

Outbound-2 and Inbound-2.

Purely for visual purposes, the plots of each of these trajectories can be found in Fig. 6.1, Fig. 6.2, and Fig. 6.3, respectively. The results are not given here in the synodic frame as they mostly look extremely similar, however these can be found in Appendix B. Furthermore, a summary of the results of each vehicle can be seen in Table 6.1

Interestingly, even between the inbound and outbound cycler trajectories, the characterstics of the manifold-to-cycler trajectory remain very similar throughout the solution space. The main difference lies in the launch date $\left(\theta_{z}\right)$ which is relatively obvious, but all other parameters linger within the same range. This is likely due 


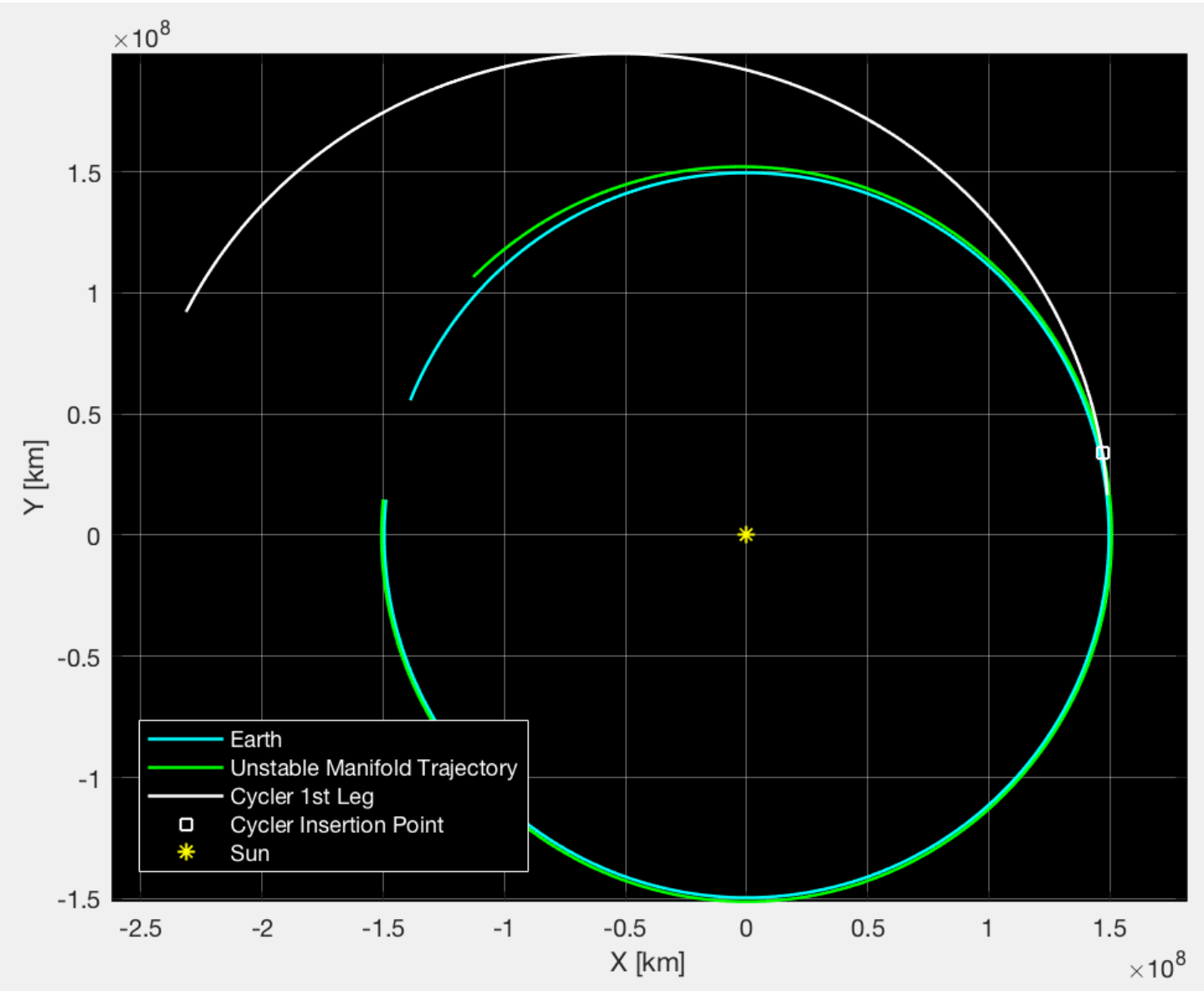

Figure 6.2: Outbound vehicle 2 results

Table 6.1: Manifold to cycler insertion results for various vehicles

\begin{tabular}{c|cccccc}
\hline & $\theta_{z}[\mathrm{rad}]$ & $\Delta V[\mathrm{~km} / \mathrm{s}]$ & $\Delta V_{\text {total }}[\mathrm{km} / \mathrm{s}]$ & $V_{\infty}[\mathrm{km} / \mathrm{s}]$ & $d_{c}[\mathrm{~km}]$ & TOF [days] \\
\hline Outbound-1 & 0.7632 & 3.02 & 5.77 & 4.01 & 132 & 298 \\
Inbound-1 & -0.4909 & 3.15 & 5.90 & 3.33 & 286 & 248 \\
Outbound-2 & 3.0434 & 2.97 & 5.72 & 4.52 & 841 & 265 \\
Inbound-2 & 5.4978 & 3.09 & 5.84 & 4.29 & 137 & 332 \\
\hline
\end{tabular}




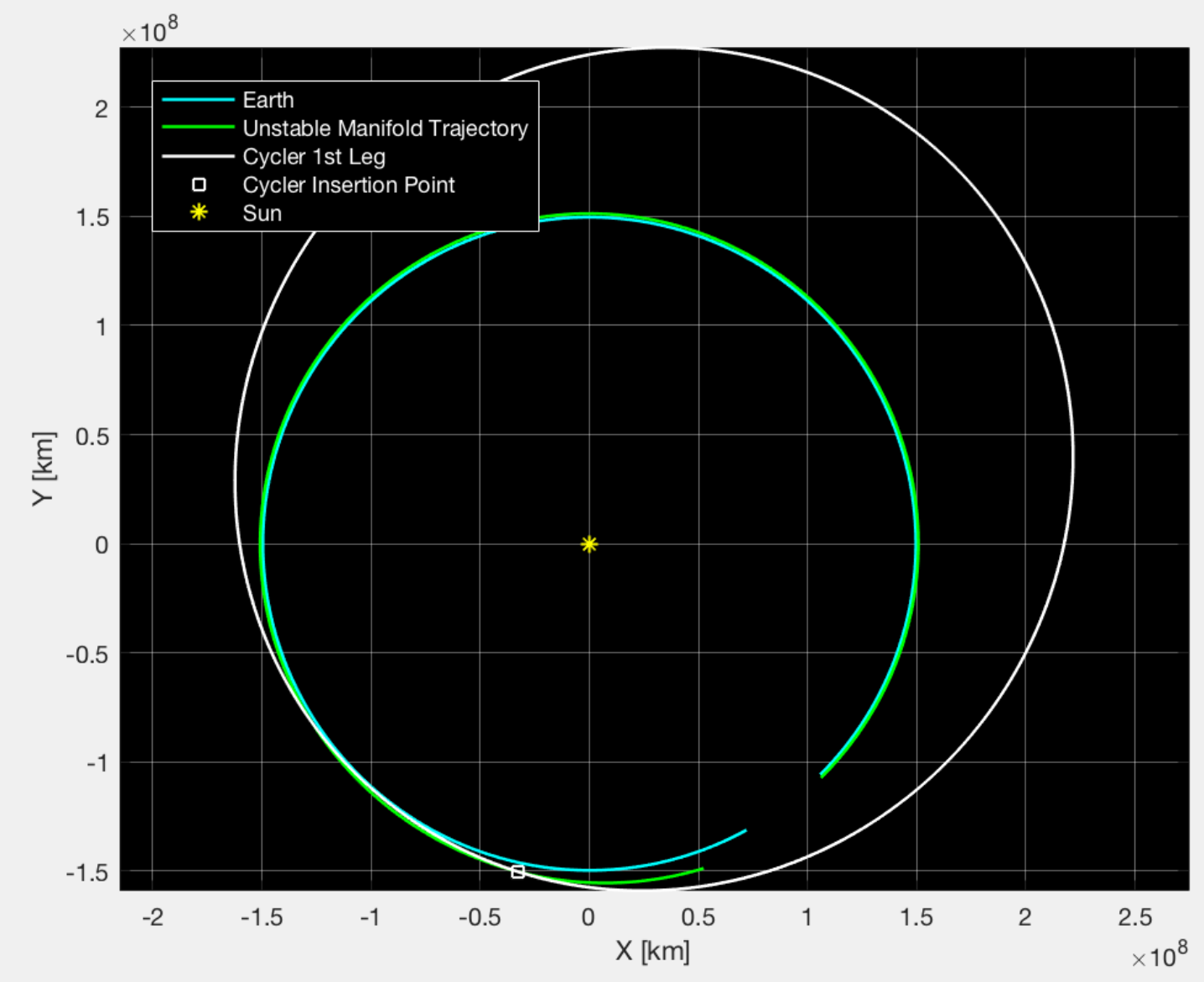

Figure 6.3: Inbound vehicle 2 results 
to the fact that the manifold is unchanging, even with launch date; and likewise the shape of the cycler trajectory is relatively similar, even though we are using the ephemeris model. Similar results between each are to be expected, but the affinity between the inbound and outbound cyclers is quite surprising.

As for the total $\Delta V$ required for each trajectory, no value can compete with the initial $V_{\infty}$ at Earth. While unfortunate in the sense that this means the mission is impractical to begin in this manner, the feasibility has still been demonstrated. While unstable manifolds can be used to burn onto cycler trajectories for less fuel than a normal burn from Earth, the burn from the parking orbit to the halo orbit is too large to allow for any sort of consideration.

A more feasible aspect of this method may lie in the possibility that the cycler vehicle can somehow start in a halo orbit or be built there via many smaller missions to the libration point. This is not so far fetched since many future missions have been proposed that involve building a vehicle in space rather than on the ground, not to mention that the International Space Station (ISS) was built in this manner. In the case where only the halo orbit is considered, this method is most certainly viable for saving on $\Delta V$ cost and should be considered for future missions. 


\section{Chapter 7}

\section{CONCLUSIONS}

Mars is still one of the most fascinating destinations for this era's astronauts, and the cycler trajectory provides a very practical and elegant solution to getting astronauts there and back as efficiently as possible. Because getting the Mars is still expensive in terms of $\Delta V$, a solution was proposed that would allow the use of manifolds to reduce mission cost.

While a few cycler trajectories were analyzed, the S1L1-B was chosen because of its capacity to perform ballistically as well as its ability to transfer to the ephemeris model of the solar system. The verification of cycler trajectories in the ephemeris model was demonstrated, which proved useful for generating further cycler trajectories without the use of an optimizer. It was also shown that both the inbound and outbound cycler trajectories are feasible and necessary for agile transport to and from Mars.

This work most importantly explored the possibility of the potential use of trajectories on a manifold to connect to a cycler trajectory, with a secondary emphasis on reducing mission cost. The effects of the CR3BP continue to provide interesting solutions to many orbital problems and the potential connection to cycler trajectories can provide more insight into their vast amounts of uses.

While the maneuver from manifold to cycler is technically possible, the necessity to burn from a parking orbit around Earth to a halo orbit requires an excess amount of $\Delta V$ not present in a direct burn sequence. This reduces the practicality of this mission type as the secondary goal was the reduce the $\Delta V$ cost associated with a cycler trajectory insertion burn. However, it was demonstrated that if the vehicle were to begin in the halo orbit automatically and burn from there, a significant amount of 
fuel can be saved.

Overall, this work was effective in establishing the ground work for the connection between manifolds and cycler trajectories. While only the Earth-Sun L2 point and the Earth-Mars cycler were considered, the feasibility of this mission type was demonstrated. However, much more analysis should be done in order to optimize the solutions further as well as expand the solution space to other libration points and cycler trajectories. Further work is also discussed in the following chapter. 


\section{Chapter 8}

\section{FUTURE WORK}

Due to the shear scope of this project, there were many aspects that could not be considered. This is largely due to the fact that two very distinct concepts - those being cycler trajectories and manifolds - were analyzed in such a way that described a possible connection between the two, rather than an in-depth analysis on either. As a result, there are many things that can be done to improve not only the results of this work, but also the nature of it.

\subsection{Cycler Trajectories}

One of the most obvious areas to expand upon is determining which leg of a generated cycler trajectory is the best to leave on. Because the cycler can technically start at any trajectory that begins at Earth, any of these are valid departure points. This can allow for another optimization parameter when constructing the manifold.

Another area to expand upon would be a more rapid approach to generating the cycler trajectory in the first place. While robust, the method developed in this work is slow-pace and does not allow for much in the way of optimization for certain parameters. Adjusting for this may allow for the cycler to be optimized for the manifold itself, rather than the other way around.

The last area of improvement to the cycler trajectory would be to expand the solution space to include other types of cyclers rather than only the S1L1-B. It may be shown that just because the cycler is ballistic, doesn't mean that it will carry the best results. 


\subsection{Manifolds}

A clear improvement in the way of manifolds is expanding to the other libration points, especially the Earth-Sun L1 point, but more generally to include the EarthMoon libration points as well. This could give insight as to how various libration

points operate, especially in terms of relative velocity to the cycler trajectory, which is the most important aspect of cycler insertion. One might even be audacious enough to use halo to halo manifold transfers from Earth to Mars rather than the flyby system of the cycler trajectory.

Another extension would be to change the size of the halo orbit within the optimization, that is to say, optimize the stable and unstable manifolds at the same time. This may allow for a very desirable trajectory to appear that was previously hidden from the solution space.

The final advancement would be to drastically increase the fidelity of the model and allow for much more accurate representations of the concepts to be calculated and shown. This can be done very simply through increasing the calculation accuracy as well as the amount of usable points on the cycler and manifold vectors. 


\section{BIBLIOGRAPHY}

[1] Aldrin Mars Cycler. https://buzzaldrin.com/spacevision/rocket_science/aldrin-mars-cycler/. Accessed: 2018-03-20.

[2] Cassini Legacy: 1997-2017. https://saturn.jpl.nasa.gov/mission/gravity-assists/. Accessed: 2018-05-13.

[3] Circular Restricted Three-Body Problem. Interplanetary Mission Design.

[4] Grand Theft Pluto. https://science.nasa.gov/science-news/science-atnasa/2007/26feb_grandtheft. Accessed: 2018-05-13.

[5] Gravity assist. http://www.planetary.org/blogs/guestblogs/2013/20130926-gravity-assist.html. Accessed: 2018-05-13.

[6] ISEE-3. https://nssdc. gsfc . nasa.gov/nmc/spacecraftDisplay. do?id=1978-079A. Accessed: 2018-03-26.

[7] JPL Planetary and Lunar Ephemerides. https://ssd.jpl.nasa.gov/?planet_eph_export. Accessed: 2018-05-13.

[8] SNOPT. http://www.sbsi-sol-optimize.com/asp/sol_product_snopt.htm. Accessed: 2018-05-13.

[9] B. Aldrin. Cyclic Trajectory Concepts. In Interplanetary Rapid Transit (IRT) Study. Aerospace Systems Group, 1985. 
[10] J. J. M. Gerard Gomez and M. W. Lo. Libration Point Orbits and Applications - Proceedings of the Conference. World Scientific, 2003.

[11] D. Izzo. Revisiting Lambert's Problem. Technical report, European Space Agency, Noordwijk AZ 2201, 2014.

[12] B. V. Jonathan Brown, Jeremy Peterson and W. Yu. Seasonal Variations of the James Webb Space Telescope Orbital Dyanmics. Technical report, Mission Engineering and Technologies Division, Lanham MD 20706, 2015.

[13] T. M. . J. M. Longuski and D. V. Byrnes. Analysis of a Broad Class of Earth-Mars Cycler Trajectories. Technical report, Purdue University and Jet Propulsion Laboratory, West Lafayette IN 47907 and Pasadena CA 91109, 2002.

[14] T. McConaghy and J. M. Longuski. Analysis of Various Two Synodic Period Earth-Mars Cycler Trajectories. Technical report, Purdue University, West Lafayette IN 47907, 2002.

[15] T. T. McConaghy. personal communication.

[16] M. R. Roussel. Invariant Manifolds. Technical report, 2005.

[17] M. Rund. Numerical Halo Orbit Computation. Cal Poly San Luis Obispo, 3 2018.

[18] R. P. Russell and C. A. Ocampo. A Systematic Method for Constructing Earth-Mars Cyclers Using Direct Return Trajectories. Technical report, The University of Texas at Austin, Austin TX 78172, 2004.

[19] N. Shupe. Earth-Mars Cycler Trajectories. ASEN5050 Research Paper.

[20] V. Szebehely. Theory of Orbits: The Restricted Problem of Three Bodies. Academic Press, 1967. 


\section{APPENDICES}

Appendix A

\section{CYCLER TRAJECTORY EXTRAS}

\section{A.1 Aldrin Cycler Computation}

I first begin by finding the period of Earth's Orbit through

$$
P_{e}=2 \pi \sqrt{\frac{a_{e}^{3}}{\mu_{s}}}
$$

where $a_{e}$ is the semi-major axis of Earth and $\mu_{s}$ is the standard gravitational parameter of the sun. I then find the period of Mars' orbit, $P_{m}$ through the definition of the synodic period, $S$.

$$
\frac{1}{S}=\frac{1}{P_{e}}-\frac{1}{P_{m}}
$$

where, $S=2 \frac{1}{7}$. By setting $n=1$ rev, I find the positions of Earth at the start and finish of one cycle as

$$
\begin{aligned}
R_{0} & =a_{e}[1,0,0] \\
R_{2 \frac{1}{7}} & =a_{e}[\cos (2 \pi n S), \sin (2 \pi n S), 0]
\end{aligned}
$$

Using the Izzo-Gooding Lambert's solution method, a multi-rev solution for the corresponding velocity vectors is found, namely $V_{0}$ and $V_{2 \frac{1}{7}}$. $R_{0}$ and $V_{0}$ are then propagated through and ODE solver, where the accelerations is defined by

$$
A=\frac{-\mu_{s}}{\|R\|^{3} R}
$$

This outputs the position and velocity of the cycler vehicle, $R_{c}$ and $V_{c}$ respectively, over the duration of the trajectory. The encounter position of Mars, $R_{m}$ is then analyzed graphically. 


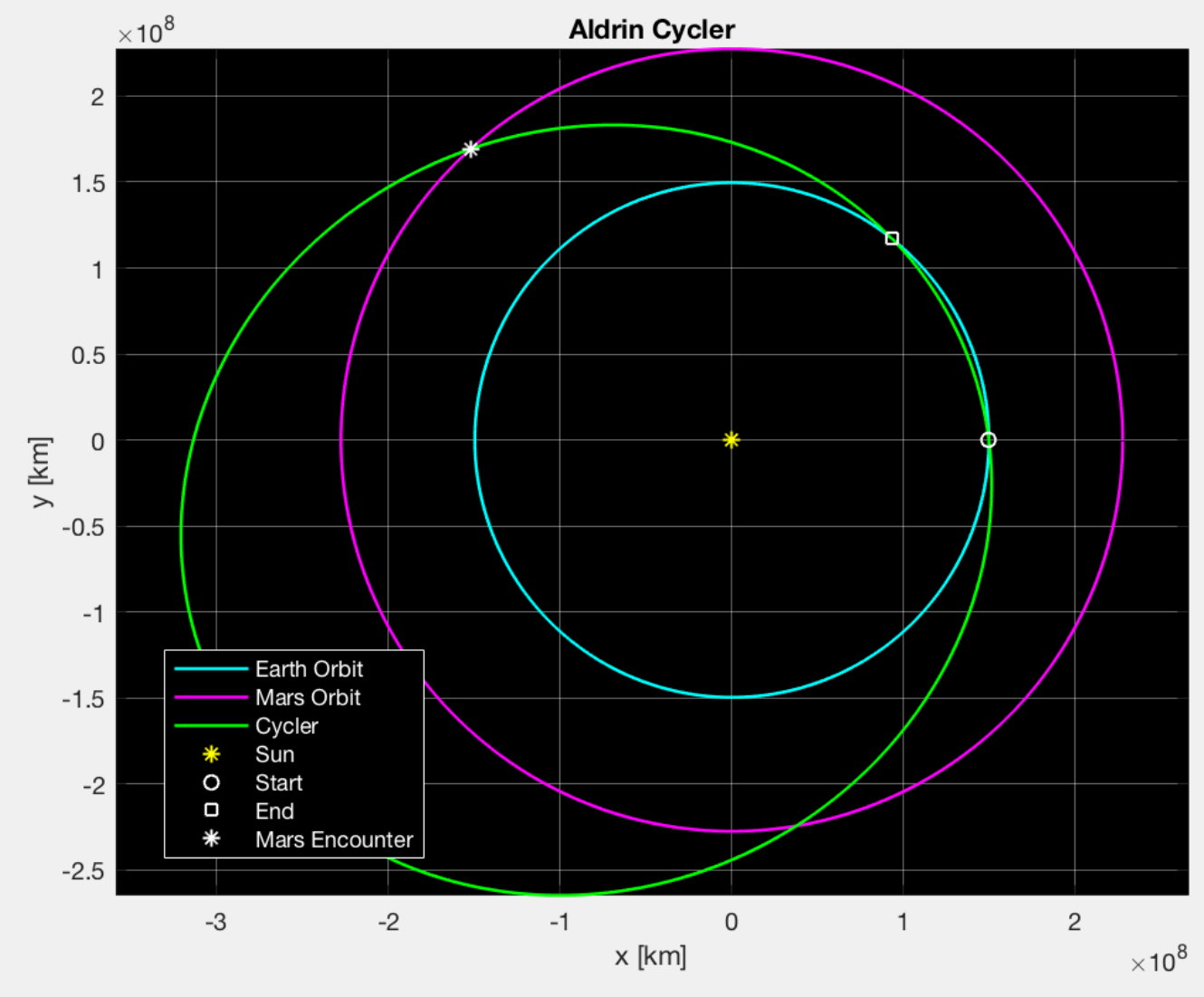

Figure A.1: Aldrin cycler results for first trajectory

My results can be summarized as follows with $a_{e}=149598023$ :

$$
\begin{aligned}
R_{0} & =a_{e}[1,0,0] \\
R_{2 \frac{1}{7}} & =a_{e}[0.6235,0.7818,0] \\
R_{m} & =a_{e}[-1.0163,0.2505,0]
\end{aligned}
$$

These and the shape of the cycler are also graphically represented in Fig. A.1.

\section{A.2 S1L1-B Cycler Computation (Circular Model)}

The computation starts the same as the Aldrin except that now $n=2$. Now I create a vector of various values of $\tau \in\{S, 2 S\}$ which represents the intermediate encounter 
time with Earth. I then calculate the position of Earth at each of these encounter times through

$$
R_{\tau}=a_{e}[\cos (2 \pi \tau), \sin (2 \pi \tau), 0]
$$

I then calculate two separate Lambert's solutions for each tau from $0 \rightarrow \tau$ and $\tau \rightarrow 4 \frac{2}{7}$. Both use a multi-rev solution but the first transfer is left branch and long way, while the second is right branch and short way. This generates the velocity vectors, $V_{0}, V_{\tau 1}$, $V_{\tau 2}$, and $V_{4 \frac{2}{7}}$. Where $V_{\tau 1}$ and $V_{\tau 2}$ occur at the same time.

In order to find the ballistic trajectory the $v_{\infty}$ at $\tau 1$ and $\tau 2$ must be calculated through the following equation:

$$
v_{\infty}=\left\|V_{\tau}-V_{e}\right\|
$$

where $V_{e}$ is the velocity of Earth at the corresponding point and can be calculated by

$$
V_{e}=\hat{u}_{t} \sqrt{\mu\left(\frac{2}{\left\|R_{\tau}\right\|}-\frac{1}{a_{e}}\right)}
$$

where $\hat{u}_{t}$ is the unit vector perpendicular to, and in plane with, the position vector, $R_{\tau}$. I then find the minimum of $\left\|v_{\infty 1}-v_{\infty 2}\right\|$. At the time, $\tau$ where this is zero, a ballistic trajectory occurs. My results for the S1L1-B are as follows:

$$
\begin{aligned}
R_{0} & =a_{e}[1,0,0] \\
R_{2.8277} & =a_{e}[0.4688,-0.8833,0] \\
R_{4 \frac{2}{7}} & =a_{e}[-0.2225,0.9749,0] \\
R_{m} & =a_{e}[-0.5192,1.4292,0]
\end{aligned}
$$

A diagram of the shape of the trajectory can also be found in Fig. A.2.

\section{A.3 Extra Itineraries}

The first itinerary given in Table A.1 is the inbound itinerary given to me by Troy McConaghy and used a basis for the inbound cycler trajectory shape. 


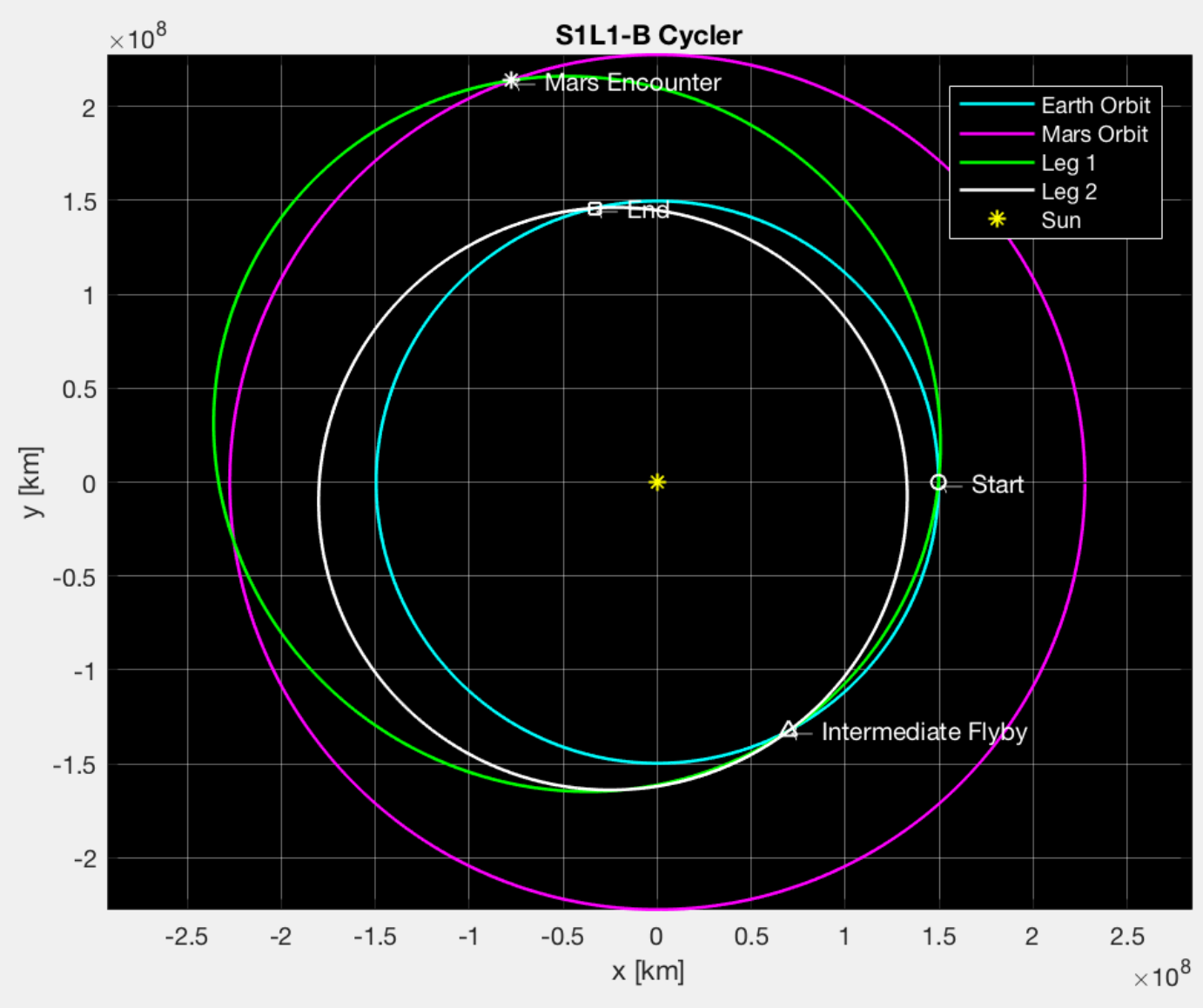

Figure A.2: S1L1-B results for legs 1 and 2 
Table A.1: Example Inbound Cycler Itinerary (T. Troy McConaghy)

\begin{tabular}{|c|c|c|c|c|c|c|c|}
\hline \multicolumn{8}{|c|}{ Inbound Cycler Vehicle (Troy McConaghy) } \\
\hline Encounter & Fly-By Planet & Year & Month & Day & $V \infty$ & $\begin{array}{l}\text { Closest } \\
\text { Approach } \\
\text { Distance }\end{array}$ & Leg, TOF \\
\hline$\#$ & $P$ & yyyy & $\mathrm{mm}$ & $d d$ & $\mathrm{~km} / \mathrm{s}$ & $\mathrm{km}$ & days \\
\hline 0 & Earth & 2005 & 4 & 1 & 3.33 & -- & -- \\
\hline 1 & Mars & 2007 & 10 & 5 & 7.25 & 12140 & 918 \\
\hline 2 & Earth & 2008 & 2 & 15 & 6.22 & 44630 & 133 \\
\hline 3 & Earth & 2009 & 8 & 7 & 6.23 & 19040 & 539 \\
\hline 4 & Mars & 2011 & 11 & 5 & 4.78 & 6710 & 820 \\
\hline 5 & Earth & 2012 & 4 & 30 & 7.05 & 29830 & 177 \\
\hline 6 & Earth & 2013 & 10 & 24 & 7.05 & 24830 & 542 \\
\hline 7 & Mars & 2016 & 1 & 8 & 2.75 & 9870 & 805 \\
\hline 8 & Earth & 2016 & 8 & 13 & 4.15 & 13410 & 218 \\
\hline 9 & Earth & 2018 & 1 & 25 & 4.17 & 22550 & 530 \\
\hline 10 & Mars & 2020 & 8 & 20 & 7.19 & 10440 & 938 \\
\hline 11 & Earth & 2020 & 12 & 6 & 4.69 & 31100 & 108 \\
\hline 12 & Earth & 2022 & 5 & 22 & 4.67 & 16020 & 532 \\
\hline 13 & Mars & 2024 & 10 & 16 & 6.64 & 3854 & 877 \\
\hline 14 & Earth & 2025 & 3 & 11 & 6.72 & 40750 & 146 \\
\hline 15 & Earth & 2026 & 9 & 3 & 6.71 & 22390 & 541 \\
\hline 16 & Mars & 2028 & 11 & 11 & 3.79 & 10580 & 800 \\
\hline 17 & Earth & 2029 & 5 & 29 & 6.19 & 25610 & 199 \\
\hline 18 & Earth & 2030 & 11 & 18 & 6.18 & 30640 & 539 \\
\hline 19 & Mars & 2033 & 4 & 7 & 3.77 & 15350 & 870 \\
\hline 20 & Earth & 2033 & 9 & 16 & 3.87 & 10950 & 162 \\
\hline 21 & Earth & 2035 & 2 & 27 & 3.84 & 24270 & 529 \\
\hline 22 & Mars & 2037 & 9 & 8 & 7.71 & 678 & 924 \\
\hline 23 & Earth & 2037 & 12 & 13 & 9.12 & -- & 96 \\
\hline
\end{tabular}


Table A.2: Second generated outbound cycler trajectory itinerary

\begin{tabular}{|c|c|c|c|c|c|c|c|}
\hline \multicolumn{8}{|c|}{ Outbound Cycler Vehicle 3} \\
\hline Encounter & Fly-By Planet & Year & Month & Day & $V_{\infty}$ & $\begin{array}{l}\text { Closest } \\
\text { Approach } \\
\text { Distance }\end{array}$ & Leg, TOF \\
\hline$\#$ & $P$ & yyyy & $\mathrm{mm}$ & $d d$ & $\mathrm{~km} / \mathrm{s}$ & $\mathrm{km}$ & days \\
\hline 1 & Earth & 2005 & 9 & 9 & 5.32 & -- & -- \\
\hline 2 & Mars & 2006 & 3 & 3 & 3 & 9960 & 175 \\
\hline 3 & Earth & 2008 & 6 & 9 & 6.92 & 20500 & 830 \\
\hline 4 & Earth & 2009 & 12 & 3 & 6.93 & 31200 & 542 \\
\hline 5 & Mars & 2010 & 6 & 6 & 4.31 & 17800 & 185 \\
\hline 6 & Earth & 2012 & 8 & 24 & 6.42 & 26500 & 809 \\
\hline 7 & Earth & 2014 & 2 & 14 & 6.43 & 41500 & 540 \\
\hline 8 & Mars & 2014 & 7 & 3 & 7.14 & 12200 & 138 \\
\hline 9 & Earth & 2016 & 12 & 9 & 4.01 & 27700 & 890 \\
\hline 10 & Earth & 2018 & 5 & 22 & 4.03 & 19900 & 530 \\
\hline 11 & Mars & 2018 & 9 & 15 & 6.47 & 11600 & 115 \\
\hline 12 & Earth & 2021 & 4 & 6 & 4.61 & 23000 & 934 \\
\hline 13 & Earth & 2022 & 9 & 20 & 4.59 & 14800 & 532 \\
\hline 14 & Mars & 2023 & 5 & 1 & 2.77 & 7590 & 223 \\
\hline 15 & Earth & 2025 & 7 & 2 & 7.08 & 23900 & 793 \\
\hline 16 & Earth & 2026 & 12 & 26 & 7.09 & 35200 & 542 \\
\hline 17 & Mars & 2027 & 6 & 14 & 5.26 & 13800 & 170 \\
\hline 18 & Earth & 2029 & 9 & 21 & 5.78 & 26800 & 830 \\
\hline 19 & Earth & 2031 & 3 & 12 & 5.78 & 39000 & 537 \\
\hline 20 & Mars & 2031 & 7 & 15 & 7.7 & 10600 & 125 \\
\hline 21 & Earth & 2034 & 1 & 15 & 3.78 & 23000 & 915 \\
\hline 22 & Earth & 2035 & 6 & 28 & 3.76 & 9630 & 529 \\
\hline 23 & Mars & 2035 & 11 & 13 & 4.68 & 15700 & 138 \\
\hline 24 & Earth & 2038 & 5 & 8 & 5.54 & -- & 907 \\
\hline
\end{tabular}

This next itinerary in Table A.2 was found by me by adjusting the launch date of the itinerary in Table 2.1 slightly and running my algorithm. Interestingly, the itinerary matches the previous very well for a significant portion of the middle of the trajectory. 


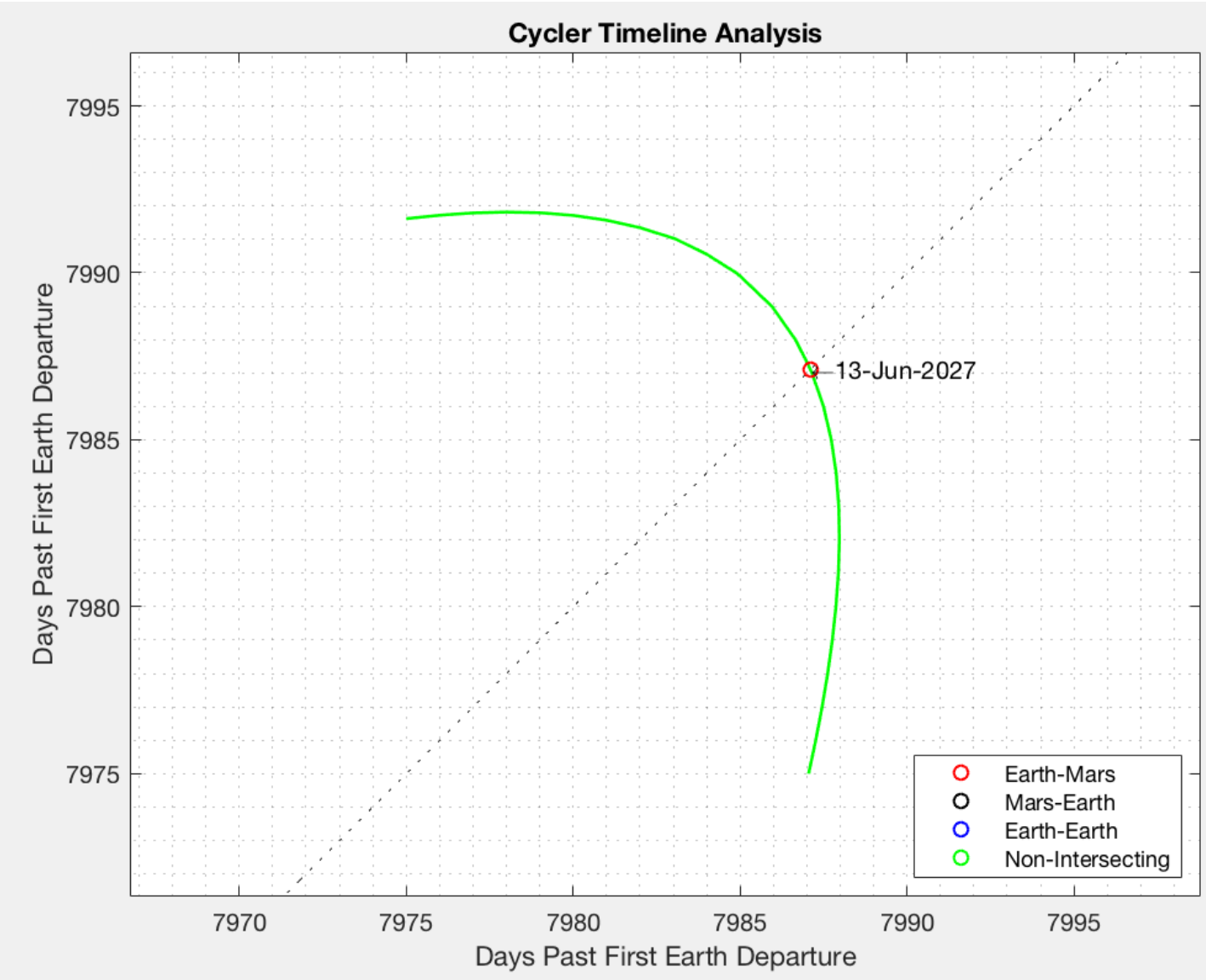

Figure A.3: Intersection of $V_{\infty}$ contour on timeline

\section{A.4 Timeline Characteristics}

As mentioned in the main portion of this work, the details of the timeline are simply the $V_{\infty}$ contours overlayed onto a dual-axis timeline. A line of $x=y$ is placed on the graph and any intersection points with the contours are valid flyby dates. Furthermore, the cycler is only complete when all flybys are valid. Fig. A.3 displays a zoomed-in visual of the timeline for one of the intersections to gain a better understanding of this graph.

Sometimes, the contour will intersect with more than one point on the timeline. This means that either flyby is perfectly valid to continue the trajectory, but only one can be used to continue the remaining computation of the cycler trajectory. An 


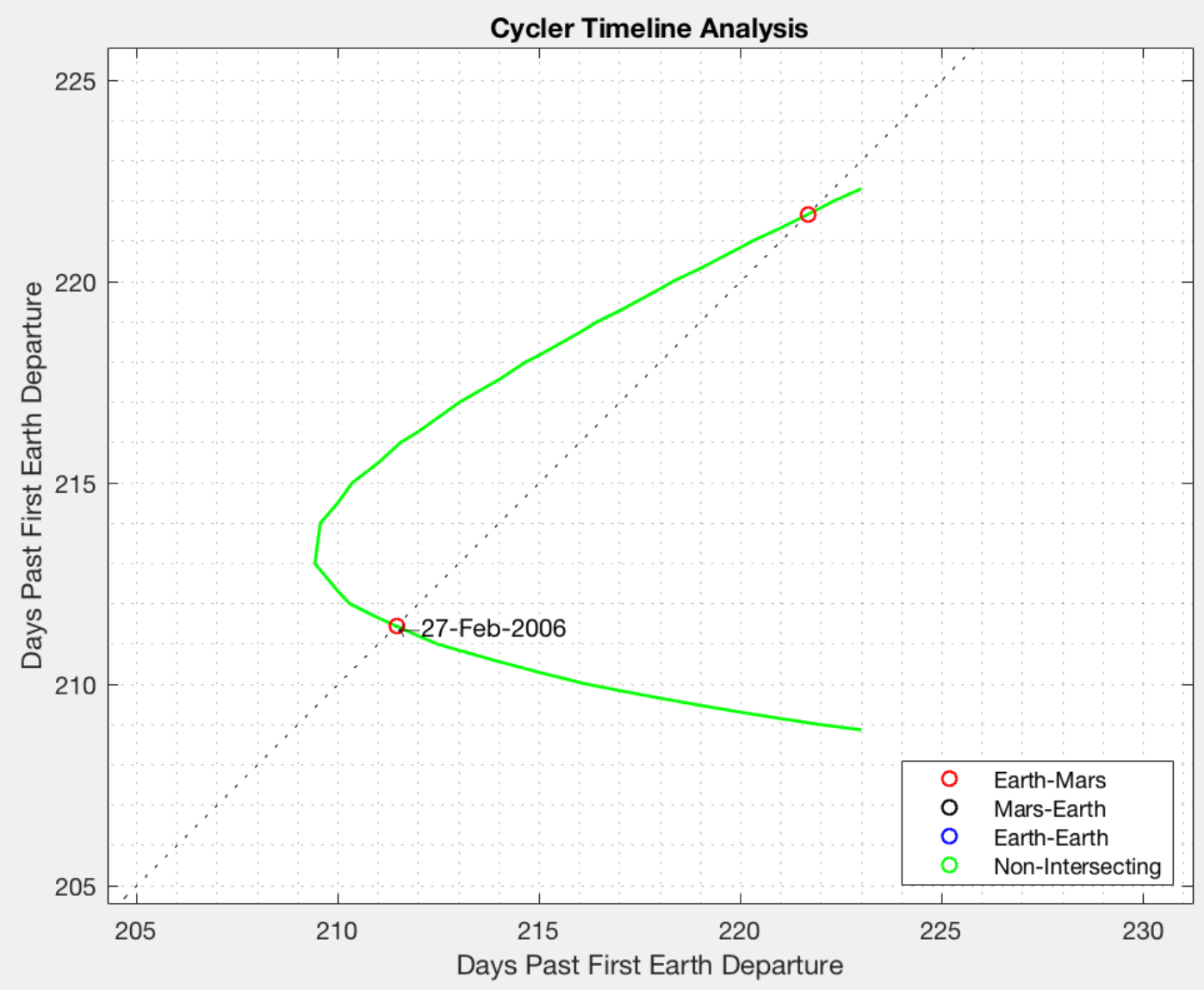

Figure A.4: Double intersection of $V_{\infty}$ contour on timeline

example of this contour can be found in Fig. A.4. 
Appendix B

MANIFOLD EXTRAS

\section{B.1 Synodic Frame Test Case Plots}

The manifold trajectories for the test case cycler trajectories are given in Fig. B.1, Fig. B.2, and Fig. B.3. These may provide some insight onto how the shape of the unstable manifold trajectory can change depending on the parameters of the cycler trajectory. 


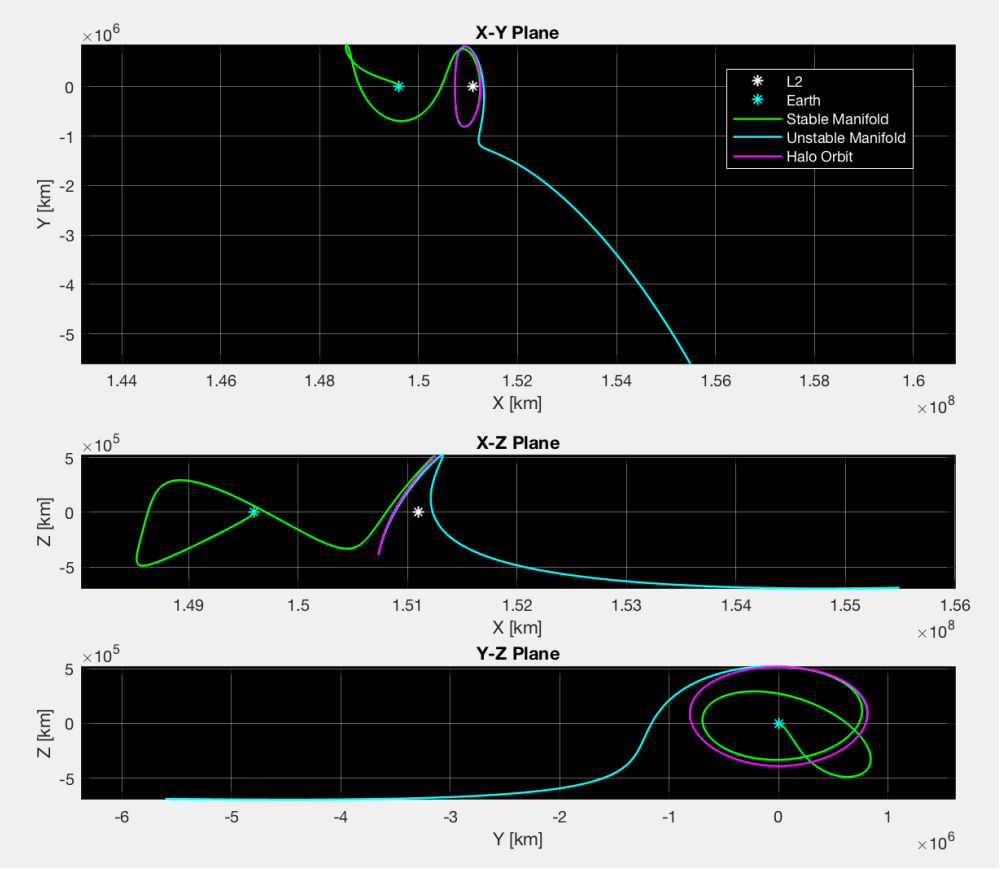

Figure B.1: Trajectory in synodic frame for Inbound-1 vehicle

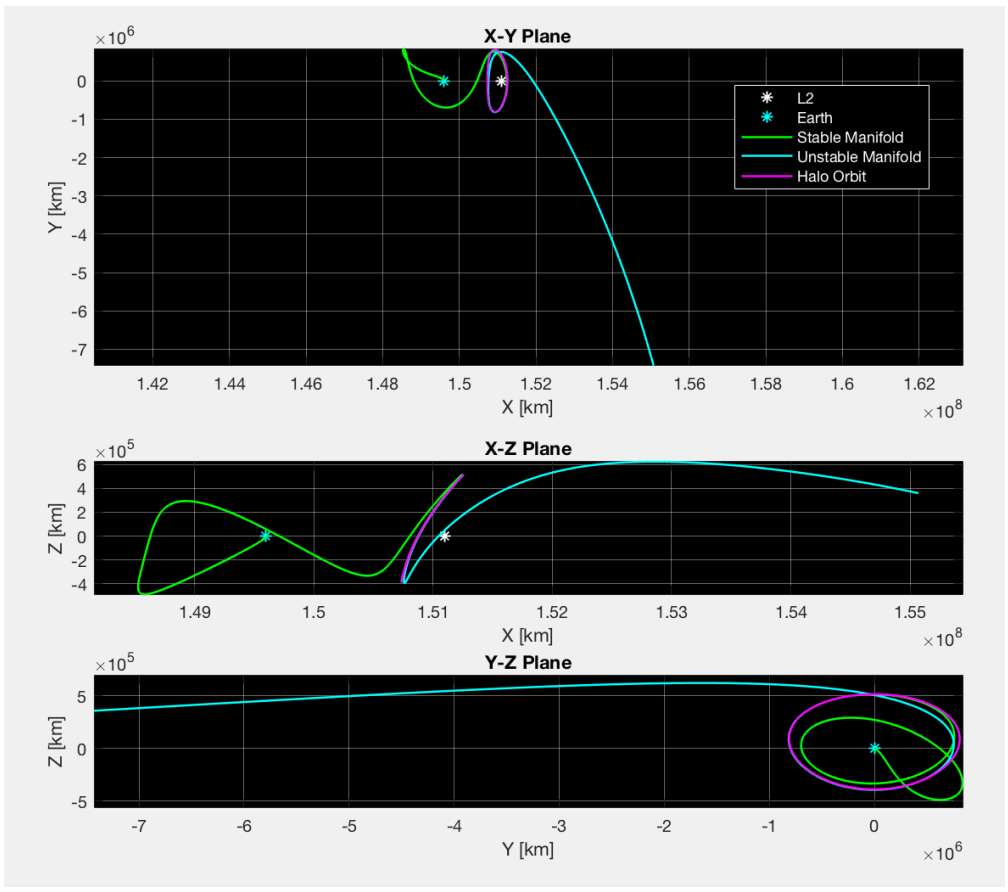

Figure B.2: Trajectory in synodic frame for Outbound-2 vehicle 

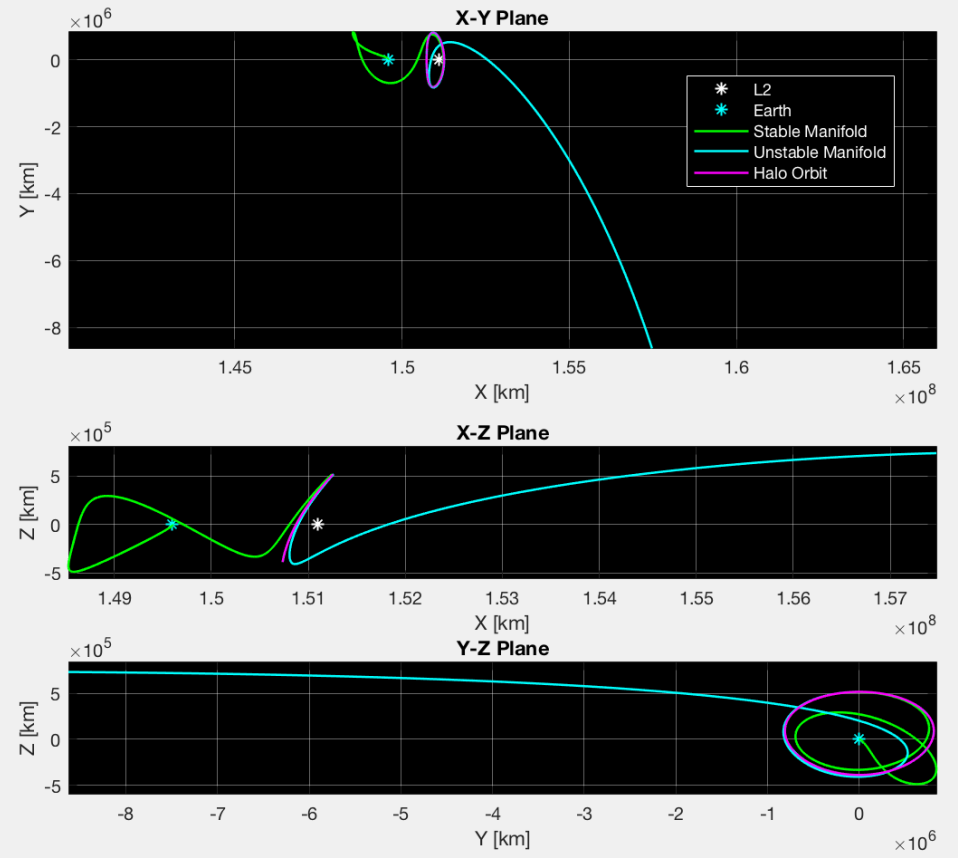

Figure B.3: Trajectory in synodic frame for Inbound-2 vehicle 